\title{
Edge Quantisation of Elliptic Operators
}

\author{
N. Dines, X. Liu, and B.-W. Schulze
}

December 2, 2004

\begin{abstract}
The ellipticity of operators on a manifold with edge is defined as the bijectivity of the components of a principal symbolic hierarchy $\sigma=\left(\sigma_{\psi}, \sigma_{\wedge}\right)$, where the second component takes values in operators on the infinite model cone of the local wedges. In the general understanding of edge problems there are two basic aspects: Quantisation of edge-degenerate operators in weighted Sobolev spaces, and verifying the ellipticity of the principal edge symbol $\sigma_{\wedge}$ which includes the (in general not explicitly known) number of additional conditions on the edge of trace and potential type. We focus here on these questions and give explicit answers for a wide class of elliptic operators that are connected with the ellipticity of edge boundary value problems and reductions to the boundary. In particular, we study the edge quantisation and ellipticity for Dirichlet-Neumann operators with respect to interfaces of some codimension on a boundary. We show analogues of the Agranovich-Dynin formula for edge boundary value problems, and we establish relations of elliptic operators for different weights, via the spectral flow of the underlying conormal symbols.
\end{abstract}

AMS-classification: 35J30, 35J70, 58J05

Keywords: Boundary value problems, edge singularities, ellipticity, spectral flow

\section{Contents}

Introduction $\quad 2$

1 Edge quantisation of boundary value problems $\quad 7$

1.1 Edge-degenerate boundary value problems . . . . . . . . . 7

1.2 Boundary value problems with parameters . . . . . . . . . 13

1.3 Edge quantisation .................... 17

1.4 Edge boundary value problems ............... 20 
2 New quantisations on closed manifolds 23

2.1 Ellipticity and reduction to the edge . . . . . . . . . . 23

2.2 Boundary value problems with fictitious edges . . . . . . . . 27

2.3 Edge quantisation of the Dirichlet-Neumann operator . . . . . . . 30

2.4 The behaviour of principal edge symbols . . . . . . . . . . . . . 35

3 Applications and Remarks $\quad 38$

3.1 The index of edge symbols in terms of the spectral flow . . . . . 38

3.2 Edge quantisation for arbitrary weights . . . . . . . . . . 42

3.3 Appendix: Proof of Theorem 2.4.3 . . . . . . . . . . . . . . 44

References $\quad 45$

\section{Introduction}

This paper is aimed at studying a category of new explicit examples in the calculus of elliptic operators on a manifold with edges. This type of investigations belongs to the analysis on manifolds with geometric singularities and is motivated by the tools that have to be developed for constructing parametrices of mixed elliptic and crack problems when the interfaces have singularities.

A 'manifold' $M$ with singularities (in the sense of 'regular' edges and corners) may be characterised by iteratively forming wedges $X^{\triangle} \times \Omega$, with a cone $X^{\triangle}:=\left(\overline{\mathbb{R}}_{+} \times X\right) /(\{0\} \times X)$ for a $C^{\infty}$ manifold $X$, and an open set $\Omega \subseteq \mathbb{R}^{q}$ as edge, then glue together such wedges to 'global' spaces, and then repeat the procedure. In this connection we impose reasonable assumptions on the transition maps of corresponding 'singular charts' that distinguish regular singularities from cuspidal ones. Parallel to this procedure we can ask the nature of ellipticity (here of boundary value problems) near the strata of the configuration in terms of a principal symbolic hierarchy, the length of which is determined by the number of iteration steps.

In the present paper we mainly focus on manifolds with edges (with or without boundary). For the case without boundary such a manifold may be obtained as a quotient space

$$
W=\mathbb{W} / \sim,
$$

where $\mathbb{W}$ is a $C^{\infty}$ manifold with boundary $\partial \mathbb{W}$ that is a fibre bundle over a $C^{\infty}$ manifold $Y$, the edge, where the fibre is a compact $C^{\infty}$ manifold $X$. If $\pi: \partial \mathbb{W} \longrightarrow Y$ is the bundle projection then the equivalence $w \sim w^{\prime}$ in (0.1) means $\pi w=\pi w^{\prime}$ for 
$w, w^{\prime} \in \partial \mathbb{W}$ or $w=w^{\prime}$ for $w, w^{\prime} \in \mathbb{W} \backslash \partial \mathbb{W}$.

The space $\mathbb{W}$ is called the stretched manifold associated with $W$. In this paper, for convenience, we assume $\partial \mathbb{W}$ to be a trivial $X$-bundle over $Y$. This assumption is not really essential, cf. [4].

A differential (or pseudo-differential) operator $A$ on $W \backslash Y$ is said to be edgedegenerate if in the splitting of variables $(r, x, y) \in \mathbb{R}_{+} \times X \times \Omega$ (locally near $\partial \mathbb{W}$ and in local coordinates $x \in \Sigma$ on $X, \Sigma \subseteq \mathbb{R}^{n}$ open, $\left.n=\operatorname{dim} X\right)$ it has an amplitude function of the form

$$
r^{-\mu} \tilde{p}(r, x, y, r \rho, \xi, r \eta)
$$

with a (classical) symbol $\tilde{p}(r, x, y, \tilde{\rho}, \xi, \tilde{\eta})$ of Hörmander's type of order $\mu$ on $\overline{\mathbb{R}}_{+} \times \Sigma \times \Omega \times \mathbb{R}_{\tilde{\rho}, \xi, \tilde{\eta}}^{1+n+q}$ (smooth in $r$ up to zero).

Examples are geometric operators (such as Laplace-Beltrami operators) with respect to a Riemannian metric of the form $d r^{2}+r^{2} g_{X}+d y^{2}$, where $g_{X}$ is a Riemannian metric on $X$.

The general (analytic) task of the edge calculus is to understand the nature of parametrices of elliptic edge-degenerate operators near the edge, to characterise elliptic regularity of solutions in suitable weighted edge Sobolev spaces under additional edge conditions (of trace and potential type) and the index of elliptic operators as explicitly as possible.

Ellipticity on a manifold with edges has much in common with elliptic boundary value problems in the usual sense. In fact, a manifold with boundary can be regarded as a special case of a manifold with edges, where the boundary plays the role of an edge and the inner normal of the (local) model cone. Operators in the case of boundary value problems are described (modulo lower order terms) by a principal symbolic hierarchy consisting of the interior and the boundary symbol, denoted by $\sigma_{\psi}$ and $\sigma_{\partial}$, respectively. The second component is operator-valued and acts in Sobolev spaces on the inner normal (standard Sobolev spaces for operators with the transmission property at the boundary, otherwise weighted spaces as in the general edge calculus).

To be more precise, if

$$
A=\sum_{j+|\alpha| \leq \mu} b_{j \alpha}(y, t) D_{y}^{\alpha} D_{t}^{j}
$$

is a differential operator in the 'half-space' $\Omega \times \overline{\mathbb{R}}_{+}$for an open set $\Omega \subseteq \mathbb{R}^{q}$, with coefficients $b_{j \alpha}(y, t) \in C^{\infty}\left(\Omega \times \overline{\mathbb{R}}_{+}\right)$, then we have

$$
\sigma_{\psi}(A)(y, t, \eta, \tau):=\sum_{j+|\alpha|=\mu} b_{j \alpha}(y, t) \eta^{\alpha} \tau^{j},
$$

$(y, t, \eta, \tau) \in T^{*}\left(\Omega \times \overline{\mathbb{R}}_{+}\right) \backslash 0$, and

$$
\sigma_{\partial}(A)(y, \eta):=\sum_{j+|\alpha|=\mu} b_{j \alpha}(y, 0) \eta^{\alpha} D_{t}^{j},
$$




$$
\sigma_{\partial}(A)(y, \eta): H^{s}\left(\mathbb{R}_{+}\right) \longrightarrow H^{s-\mu}\left(\mathbb{R}_{+}\right),
$$

$(y, \eta) \in T^{*} \Omega \backslash 0$.

Note that $A$ can also be written in the form

$$
A=r^{-\mu} \sum_{j+|\alpha| \leqq \mu} a_{j \alpha}(r, y)\left(-r \partial_{r}\right)^{j}\left(r D_{y}\right)^{\alpha}
$$

with coefficients $a_{j \alpha}(r, y) \in C^{\infty}\left(\overline{\mathbb{R}}_{+} \times \Omega\right)$; then $\sigma_{\partial}(A)(y, \eta)$ takes the form of the so called principal edge symbol

$$
\sigma_{\wedge}(A)(y, \eta):=r^{-\mu} \sum_{j+|\alpha| \leqq \mu} a_{j \alpha}(0, y)\left(-r \partial_{r}\right)^{j}(r \eta)^{\alpha} .
$$

More generally, if $X$ is a $C^{\infty}$ manifold, a differential operator $A$ on the (open stretched) wedge $X^{\wedge} \times \Omega$ for $X^{\wedge}:=\mathbb{R}_{+} \times X$ is called edge-degenerate if it has the form (0.4) with coefficients $a_{j \alpha}(r, y) \in C^{\infty}\left(\overline{\mathbb{R}}_{+} \times \Omega\right.$, Diff $\left.{ }^{\mu-(j+|\alpha|)}(X)\right)$; here $\operatorname{Diff}^{\nu}(\cdot)$ denotes the (Fréchet) space of all differential operators of order $\nu$ on the $C^{\infty}$ manifold in the brackets. We shall see below that there is a natural scale of weighted Sobolev spaces $\mathcal{K}^{s, \gamma}\left(X^{\wedge}\right)$ on the infinite stretched model cone $X^{\wedge}$ such that (0.5) induces continuous operators

$$
\sigma_{\wedge}(A)(y, \eta): \mathcal{K}^{s, \gamma}\left(X^{\wedge}\right) \longrightarrow \mathcal{K}^{s-\mu, \gamma-\mu}\left(X^{\wedge}\right)
$$

for all $s, \gamma \in \mathbb{R}$. The pair $\left(\sigma_{\psi}(A), \sigma_{\wedge}(A)\right)$ (with $\sigma_{\psi}(A)$ being the standard homogeneous principal symbol of $A$ ) is the principal symbolic hierarchy of $A$ in the sense of the edge calculus.

Recall that in boundary value problems for an elliptic operator (0.2) the extra boundary conditions (such as Dirichlet or Neumann conditions) come from filling up the (in the differential case surjective) family (0.3) of Fredholm operators to a block matrix family of isomorphisms, for $(y, \eta) \in T^{*} \Omega \backslash 0$. Similarly, for an elliptic edge-degenerate operator (0.4) we know the Fredholm property of $(0.6)$ for all $\gamma$ (except for a discrete set of 'forbidden' weights that may depend on $y$ ). The concept of ellipticity of edge problems is also to add extra edge conditions of trace and potential type, generated by a corresponding block matrix family of isomorphisms

$$
\sigma_{\wedge}(\mathcal{A})(y, \eta):=\left(\begin{array}{cc}
\sigma_{\wedge}(A) & \sigma_{\wedge}(K) \\
\sigma_{\wedge}(T) & \sigma_{\wedge}(Q)
\end{array}\right)(y, \eta): \begin{gathered}
\mathcal{K}^{s, \gamma}\left(X^{\wedge}\right) \\
\oplus \\
J_{-, y}
\end{gathered} \rightarrow \begin{gathered}
\mathcal{K}^{s-\mu, \gamma-\mu}\left(X^{\wedge}\right) \\
\oplus \\
J_{+, y}
\end{gathered}
$$

for any fixed choice of an admissible weight. Associated operators

$$
\mathcal{A}=\left(\begin{array}{ll}
A & K \\
T & Q
\end{array}\right)
$$

acting in a suitable scale of weighted Sobolev spaces then represent a category of elliptic edge problems for $A$, and $\sigma(\mathcal{A})=\left(\sigma_{\psi}(A), \sigma_{\wedge}(\mathcal{A})\right)$ is the principal symbolic 
hierarchy of the operator $\mathcal{A}$.

Note that, in contrast to the known behaviour of elliptic differential operators, in the edge calculus we need both trace and potential operators at the same time, depending on the weights. In addition it may be extremely difficult to explicitly calculate the number of extra data that complete $\sigma_{\wedge}(A)(y, \eta)$ to a family of isomorphisms (0.7).

The construction needs information on kernels and cokernels of $\sigma_{\wedge}(A)(y, \eta)$ on the infinite cone. From that point of view it is interesting to analyse sufficiently large classes of explicit examples as is done in the papers [4] for the case of closed manifolds with edges and in [19] for the case of boundary value problems. The applications of the calculus, for instance, to crack problems [13] or to mixed elliptic problems [3] lead to many other 'unexpected' difficulties. These lie partly in the complexity of phenomena that is connected with the iterative structure of the calculi and in the relevance of 'nonlinear spectral information' on subordinate conormal symbols which determines not only the admissible weights but also the number of additional edge conditions.

The present paper is focused on a number of new aspects in connection with elliptic boundary value problems. In this case we have $3 \times 3$ block matrices that contain both boundary and edge conditions, and the principal symbolic hierarchy of such an operator $\mathfrak{A}$ consists of three components:

$$
\sigma(\mathfrak{A})=\left(\sigma_{\psi}(\mathfrak{A}), \sigma_{\partial}(\mathfrak{A}), \sigma_{\wedge}(\mathfrak{A})\right) .
$$

This paper is organised as follows:

In Chapter 1 we introduce the basics on (pseudo-differential) boundary value problems on a manifold with boundary and edge that have the transmission property at the smooth part of the boundary. Close to the edges we impose the typical edge-degenerate behaviour of symbols which admits operators that are continuous in weighted edge Sobolev spaces. In the case of differential boundary value problems the quantisation is precise, i.e., they are no smoothing remainders to be neglected, while in the pseudo-differential case we employ the Mellin transform in axial direction of the model cone, combined with a kernel cut-off argument which produces holomorphic Mellin symbols. The quantisation itself is based on amplitude functions of the form (1.23) in a scenario of operator-valued symbols with 'twisted homogeneity'. Also the weighted edge Sobolev spaces are based on suitable rescalings in weighted Sobolev spaces on the infinite model cone. The main feature of our approach is that the edge boundary value problems are a generalisation of Boutet de Monvel's calculus [2], here, for the case of edge singularities on the boundary. Other elements of this 'edge algebra' of boundary value problems may be found in [13], see also [31], [32], [33].

Chapter 2 is devoted to the problem of reducing edge boundary value problems to the edge and to the boundary. In the boundary reduction we take boundary value problems with the same elliptic operator in the upper left corner but different edge and boundary conditions, and we prove an index formula of AgranovichDynin type. This compares the indices of the involved problems in terms of an 
elliptic operator on the boundary which is a 'closed' manifold with edge. In a similar spirit we reduce elliptic edge problems to the edge and obtain an analogue of the Agranovich-Dynin formula also in this case. We apply the reduction to the boundary for constructing new (compared with [4]) edge quantisations for elliptic operators on a closed manifold with 'fictitious' edges. As an example we discuss the Dirichlet-Neumann operator in more detail. The process of reducing problems to the boundary is also ilustrated in terms of principal edge symbols which gives us new explicit expressions of parametrices for classes of elliptic boundary value problems on infinite cones.

In Chapter 3 we discuss the problem to what extent our elliptic edge problems (constructed before for 'sufficiently large' weights and with the exception of discrete exceptional weights) can be understood for arbitrary weights (that may be negative and of large absolute value). This depends on the evaluation of index elements of homogeneous principal edge symbols in the $2 \times 2$ upper left corners. We express 'the number' of new edge conditions in terms of the spectral flow of subordinate conormal symbols which represents (after the results of [24]) elements of the $K$-group on the unit cosphere bundle of the edge. In a final section we illustrate the way of constructing extra edge conditions for all weights, provided that the principal conormal symbol is a bijective family on the corresponding weight line in the complex plane.

Let us finally note that the analysis on configurations with edges (and other geometric singularities) is motivated by models of mechanics, physics and the applied sciences, but also by problems of geometry and topology. Many authors have contributed under different aspects to this field. The classical theory of (pseudo-differential) boundary value problems in the sense of Vishik and Eskin [38], [39], [6], Boutet de Monvel [2] can be subsumed under the 'edge analysis'. Pseudo-differential boundary value problems are, of course, of independent value. In particular, there are interesting parameter-dependent variants, see, e.g., the work of Grubb [11]. The dependence of parameters is also crucial for the 'higher floors' of the analysis on stratified spaces, cf. [35]. As a starting point one can take the case of manifolds with conical singularities, cf. Kondratiev [14] who studied boundary value problems on such manifolds. Observe that singular integral operators in the spirit of Gohberg and Krupnik [9] can be seen as an aspect of operators on a manifold with conical singularities, cf. also the Mellin operator calculus in [6]. These relations show the long history of the analysis on 'manifolds with singularities' which cannot be reported here in detail; more references on the historical background may be found in Kondratiev and Oleynik [15], Plamenevskij [29], Kapanadze and Schulze [13]. Papers on concrete problems in domains with edges are owed by Mazja and Plamenevskij [21] and Mazja and Rossmann [22]. The pseudodifferential approach to the analysis on manifolds with edges that is used here is developed in [34]; more technicalities may be found in Egorov and Schulze [5] or in other monographs, see, e.g., [13] and the references there. In recent years the index theory and the homotopy classification of elliptic edge operators was intensively developed, see the papers of Nazaikinskij, Savin, Schulze, and Sternin [23], [24], [25], 
and the references there. There are also connections with anisotropic elliptic and parabolic boundary value problems, especially on long-time asymptotics of solutions, see [16], [17]. Another stream of new papers studies ellipticity and index on more general spaces, see, e.g., Nistor [28], Lauter and Nistor [18], Loya [20]. Spectral properties of elliptic operators on manifolds with conical singularities have been investigated by Gil, Krainer and Mendoza [7], [8], where the spectral parameter was interpreted as an edge covariable.

\section{Edge quantisation of boundary value problems}

\subsection{Edge-degenerate boundary value problems}

Let $W$ be a manifold with edge $Y$ and boundary, that is, $W$ is a topological space with a subspace $Y$, such that $W \backslash Y$ is a $C^{\infty}$ manifold with boundary, $Y$ a $C^{\infty}$ manifold, and $W$ is locally near any point $y \in Y$ modelled on a wedge $X^{\triangle} \times \Omega$, where $X$ is a (here compact) $C^{\infty}$ manifold with boundary $\partial X, n=\operatorname{dim} X$, and $\Omega \subseteq \mathbb{R}^{q}$ an open set, $q=\operatorname{dim} Y$. For convenience all manifolds in consideration are assumed to be countable unions of compact sets. In addition smooth manifolds are equipped with Riemannian metrics and associated measures. From $W$ we can pass to the double $2 W$ obtained by gluing together two copies $W \pm$ of $W$ along the common boundary (with the plus side being identified with $W$ ). Then $M:=2 W$ is a manifold with edge $Y$ and without boundary locally near $Y$ modelled on $(2 X)^{\triangle} \times \Omega$, and we have the associated stretched manifold $\mathbb{M}$ as explained in the introduction, $M=\mathbb{M} / \sim$. Denoting by $\pi: \mathbb{M} \longrightarrow M$ the canonical map to the quotient space we then set

$$
\mathbb{W}:=\pi^{-1}\left(W_{+}\right) \text {and } \mathbb{W}_{\text {reg }}:=(\mathbb{M} \backslash \partial \mathbb{M}) \cap \mathbb{W}, \mathbb{W}_{\text {sing }}:=\partial \mathbb{M} \cap \mathbb{W} .
$$

The boundary $V:=\partial(W \backslash Y) \cup Y$ is then a manifold with edge $Y$ without boundary, locally near $Y$ modelled on $(\partial X)^{\triangle} \times \Omega$, and we have an associated stretched manifold $\mathbb{V}$.

Operators near $Y$ are considered in the splitting of variables $(r, x, y) \in X^{\wedge} \times \Omega$ with the covariables $(\rho, \xi, \eta)$. We will study a category of (pseudo-differential) boundary value problems on $W \backslash Y$ for operators $A \in L_{\mathrm{cl}}^{\mu}\left((W \backslash Y)_{\text {int }}\right)$ that have the transmission property at the smooth part $\partial(W \backslash Y)$ of the boundary and are edgedegenerate near $Y$. Here $L_{\mathrm{cl}}^{\mu}(\cdot)$ denotes the space of classical pseudo-differential operators of order $\mu$ on the manifold in the brackets (i.e., with local classical symbols in the covariable $\xi$ with homogeneous components of order $\mu-j, j \in \mathbb{N}$ ). We also need the parameter-dependent variant, with parameter $\lambda \in \mathbb{R}^{l}$, denoted by $L_{\mathrm{cl}}^{\mu}\left(\cdot ; \mathbb{R}^{l}\right)$, where the local symbols are classical in $(\xi, \lambda)$ of order $\mu$.

The edge-degenerate differential operators $A$ discussed in the introduction can be 
written in the form $\mathrm{Op}_{y}(p)$ for the operator function

$$
p(y, \eta)=r^{-\mu} \sum_{j+|\alpha| \leqq \mu} a_{j \alpha}(r, y)\left(-r \partial_{r}\right)^{j}(r \eta)^{\alpha} ;
$$

here, for simplicity, we assume the coefficients $a_{j \alpha}(r, y) \in C^{\infty}\left(\overline{\mathbb{R}}_{+} \times\right.$ $\Omega$, Diff $\left.{ }^{\mu-(j+|\alpha|)}(X)\right)$ to be independent of $r$ for large $r$. Then $p(y, \eta)$ is continuous between spaces $\mathcal{K}^{s, \gamma}\left(X^{\wedge}\right)$ that are defined as follows:

First let $N:=2 X$ denote the double of $X$, defined by gluing together two copies $X_{ \pm}$of $X$ along the common boundary (we then often identify the + side with $X)$. On the infinite stretched cone $N^{\wedge}=\mathbb{R}_{+} \times N$ we have the space $L^{2}\left(N^{\wedge}\right)$ with the measure $d r d x$ and then define $\mathcal{H}^{s, 0}\left(N^{\wedge}\right)$ for $s \in \mathbb{N}$ to be the subspace of all $u(r, x) \in r^{-\frac{n}{2}} L^{2}\left(N^{\wedge}\right)$ such that $\left(r \partial_{r}\right)^{k} V^{\alpha} u(r, x) \in r^{-\frac{n}{2}} L^{2}\left(N^{\wedge}\right)$ for all $k+|\alpha| \leqq s$, for $V^{\alpha}:=V_{1}^{\alpha_{1}} \cdot \ldots \cdot V_{n}^{\alpha_{n}}, \alpha=\left(\alpha_{1}, \ldots \alpha_{n}\right)$, for arbitrary vector fields $V_{j}$ on $N$. This definition extends to arbitrary $s \in \mathbb{R}$ by duality and interpolation, and we then set $\mathcal{H}^{s, \gamma}\left(N^{\wedge}\right):=r^{\gamma} \mathcal{H}^{s, 0}\left(N^{\wedge}\right)$ for $s, \gamma \in \mathbb{R}$. Moreover, let $H_{\text {cone }}^{s}\left(N^{\wedge}\right)$ denote the subspace of all $\left.u \in H_{\text {loc }}^{s}(\mathbb{R} \times N)\right|_{\mathbb{R}_{+} \times N}$ which behave as the standard Sobolev spaces for $r \rightarrow \infty$; in particular, when $N=S^{n}$ is the unit sphere in $\mathbb{R}^{n+1}$, $H_{\text {cone }}^{s}\left(N^{\wedge}\right)$ far from $r=0$ corresponds to $H^{s}\left(\mathbb{R}^{n+1}\right)$ when $(r, x) \in \mathbb{R}_{+} \times S^{n}$ are polar coordinates in $\mathbb{R}^{n+1} \backslash\{0\}$.

In this paper a cut-off function on the half-axis is any $\omega(r) \in C_{0}^{\infty}\left(\overline{\mathbb{R}}_{+}\right)$that is equal to 1 near 0 . We then form the space

$$
\mathcal{K}^{s, \gamma}\left(N^{\wedge}\right):=\left\{\omega u+(1-\omega) v: u \in \mathcal{H}^{s, \gamma}\left(N^{\wedge}\right), v \in H_{\text {cone }}^{s}\left(N^{\wedge}\right)\right\}
$$

for any such $\omega$ (the space is independent of the choice of $\omega$ ). Observe that (1.2) is a Hilbert space for a suitable scalar product; we then have a natural identification $\mathcal{K}^{0,0}\left(N^{\wedge}\right)=r^{-\frac{n}{2}} L^{2}\left(N^{\wedge}\right)$. For $X$ itself we set $\mathcal{K}^{s, \gamma}\left(X^{\wedge}\right):=$ $\left\{\left.u\right|_{\mathbb{R}_{+} \times \operatorname{int} X}: u \in \mathcal{K}^{s, \gamma}\left(N^{\wedge}\right)\right\}$ endowed with the Hilbert space structure from the identification $\mathcal{K}^{s, \gamma}\left(X^{\wedge}\right)=\mathcal{K}^{s, \gamma}\left(N^{\wedge}\right) / \sim$ for $\left.u \sim v \Longleftrightarrow u\right|_{\mathbb{R}_{+} \times \text {int } X}=\left.v\right|_{\mathbb{R}_{+} \times \operatorname{int} X}$. As announced before, the operators (1.1) form a family of continuous maps

$$
p(y, \eta): \mathcal{K}^{s, \gamma}\left(X^{\wedge}\right) \longrightarrow \mathcal{K}^{s-\mu, \gamma-\mu}\left(X^{\wedge}\right)
$$

for all $s, \gamma \in \mathbb{R}, C^{\infty}$ dependent on $(y, \eta) \in \Omega \times \mathbb{R}^{q}$.

We will often use the fact that the spaces $\mathcal{K}^{s, \gamma}\left(N^{\wedge}\right)$ or $\mathcal{K}^{s, \gamma}\left(X^{\wedge}\right)$ are equipped with a strongly continuous group $\left\{\kappa_{\delta}\right\}_{\delta \in \mathbb{R}_{+}}$of isomorphisms when we set

$$
\left(\kappa_{\delta} u\right)(r, x)=\delta^{\frac{n+1}{2}} u(\delta r, x),
$$

$\delta \in \mathbb{R}_{+}$. In general, let $E$ be a Hilbert space equipped with a strongly continuous group $\kappa:=\left\{\kappa_{\delta}\right\}_{\delta \in \mathbb{R}_{+}}$of isomorphisms $\kappa_{\delta}: E \longrightarrow E, \kappa_{\delta} \kappa_{\delta^{\prime}}=\kappa_{\delta \delta^{\prime}}$, for all $\delta, \delta^{\prime} \in$ $\mathbb{R}_{+}$; in that case we simply say that $E$ is endowed with a group action. We then have the following so called abstract edge Sobolev spaces (modelled on the space $E$, and with 'edge' $\mathbb{R}^{q}$ ) : 
Definition 1.1.1. The abstract edge Sobolev space $\mathcal{W}^{s}\left(\mathbb{R}^{q}, E\right)$ is defined to be the completion of $\mathcal{S}\left(\mathbb{R}^{q}, E\right)$ with respect to the norm

$$
\|u\|_{\mathcal{W}^{s}\left(\mathbb{R}^{q}, E\right)}=\left\{\int\langle\eta\rangle^{2 s}\left\|\kappa_{\langle\eta\rangle}^{-1} \hat{u}(\eta)\right\|_{E}^{2} d \eta\right\}^{\frac{1}{2}}
$$

with $\hat{u}(\eta)$ being the Fourier transform of $u$ on $\mathbb{R}^{q}$.

Remark 1.1.2. Clearly the space $\mathcal{W}^{s}\left(\mathbb{R}^{q}, E\right)$ depends on the choice of the group action $\left\{\kappa_{\delta}\right\}_{\delta \in \mathbb{R}_{+}}$on E. If necessary we write $\mathcal{W}^{s}\left(\mathbb{R}^{q}, E\right)_{\kappa}$ for the corresponding edge space. Observe that $\kappa^{\prime}:=\left\{\kappa_{\delta}^{\prime}\right\}_{\delta \in \mathbb{R}_{+}}, \kappa_{\delta}^{\prime}:=\delta^{\nu} \kappa_{\delta}, \delta \in \mathbb{R}_{+}$, defines another group action on $E$. We then have $\mathcal{W}^{s}\left(\mathbb{R}^{q}, E\right)_{\kappa}=\mathcal{W}^{s+\nu}\left(\mathbb{R}^{q}, E\right)_{\kappa^{\prime}}$.

Example 1.1.3. For $E:=\mathcal{K}^{s, \gamma}\left(N^{\wedge}\right)$ we thus obtain weighted edge Sobolev spaces

$$
\mathcal{W}^{s, \gamma}\left(N^{\wedge} \times \mathbb{R}^{q}\right):=\mathcal{W}^{s}\left(\mathbb{R}^{q}, \mathcal{K}^{s, \gamma}\left(N^{\wedge}\right)\right),
$$

based on the group action (1.4). In a similar manner we define $\mathcal{W}^{s, \gamma}\left(X^{\wedge} \times \mathbb{R}^{q}\right)$. Note that the operator of restriction $\left.u(r, x) \rightarrow u(r, x)\right|_{\mathbb{R}_{+} \times \partial X}$ first defined on $u \in$ $C_{0}^{\infty}\left(X^{\wedge}\right)$, extends to a continuous operator

$$
\mathcal{W}^{s, \gamma}\left(X^{\wedge} \times \mathbb{R}^{q}\right) \longrightarrow \mathcal{W}^{s-\frac{1}{2}, \gamma-\frac{1}{2}}\left((\partial X)^{\wedge} \times \mathbb{R}^{q}\right)
$$

for every $s>\frac{1}{2}, \gamma \in \mathbb{R}$. Also here the edge spaces are defined in terms of the " $n$ atural' group actions, connected with $n=\operatorname{dim} X$ and $n-1=\operatorname{dim} \partial X$, respectively.

Parallel to the abstract edge Sobolev spaces there is a useful notion of operatorvalued symbols with 'twisted homogeneity'.

Definition 1.1.4. (i) Let $E$ and $\tilde{E}$ be Hilbert spaces with group actions $\left\{\kappa_{\delta}\right\}_{\delta \in \mathbb{R}_{+}}$and $\left\{\tilde{\kappa}_{\delta}\right\}_{\delta \in \mathbb{R}_{+}}$, respectively. Then $S^{\mu}\left(\Omega \times \mathbb{R}^{q} ; E, \tilde{E}\right)$ denotes the set of all $a(y, \eta) \in C^{\infty}\left(\Omega \times \mathbb{R}^{q}, \mathcal{L}(E, \tilde{E})\right)$ such that

$$
\sup _{(y, \eta) \in K \times \mathbb{R}^{q}}\langle\eta\rangle^{-\mu+|\beta|}\left\|\tilde{\kappa}_{\langle\eta\rangle}^{-1}\left\{D_{y}^{\alpha} D_{\eta}^{\beta} a(y, \eta)\right\} \kappa_{\langle\eta\rangle}\right\|_{\mathcal{L}(E, \tilde{E})}
$$

is finite for every $\alpha, \beta \in \mathbb{N}^{q}$ and $K \subset \subset \Omega$.

(ii) The subspace $S_{\mathrm{cl}}^{\mu}\left(\Omega \times \mathbb{R}^{q} ; E, \tilde{E}\right)$ of classical symbols is based on components $a_{(\mu-j)}(y, \eta) \in C^{\infty}\left(\Omega \times\left(\mathbb{R}^{q} \backslash\{0\}\right), \mathcal{L}(E, \tilde{E})\right)$ of twisted homogeneity

$$
a_{(\mu-j)}(y, \delta \eta)=\delta^{\mu-j} \tilde{\kappa}_{\delta} a_{(\mu-j)}(y, \eta) \kappa_{\delta}^{-1},
$$

$\delta \in \mathbb{R}_{+} ;$more precisely, a classical symbol is an asymptotic sum of terms $\chi(\eta) a_{(\mu-j)}(y, \eta), j \in \mathbb{N}$, for any excision function $\chi$ in $\mathbb{R}^{q}$, modulo a symbol in $S^{-\infty}\left(\Omega \times \mathbb{R}^{q} ; E, \tilde{E}\right):=\bigcap_{\mu \in \mathbb{R}} S^{\mu}\left(\Omega \times \mathbb{R}^{q} ; E, \tilde{E}\right)$. 
If a relation is valid in the classical or non-classical case, we also write subscript '(cl)'. The subspaces of $y$-independent symbols are denoted by $S_{(\mathrm{cl})}^{\mu}\left(\mathbb{R}^{q} ; E, \tilde{E}\right)$.

Remark 1.1.5. The spaces $S_{(\mathrm{cl})}^{\mu}\left(\Omega \times \mathbb{R}^{q} ; E, \tilde{E}\right)$ depend on the choice of the group actions $\kappa$ and $\tilde{\kappa}$ on $E$ and $\tilde{E}$, respectively. If necessary we write $S_{(\mathrm{cl})}^{\mu}(\Omega \times$ $\left.\mathbb{R}^{q} ; E, \tilde{E}\right)_{\kappa, \tilde{\kappa}}$. Setting $\kappa^{\prime}:=\left\{\delta^{\nu} \kappa_{\delta}\right\}_{\delta \in \mathbb{R}_{+}}$and $\tilde{\kappa}^{\prime}:=\left\{\delta^{\tilde{\nu}} \tilde{\kappa}_{\delta}\right\}_{\delta \in \mathbb{R}_{+}}$we obtain

$$
S_{(\mathrm{cl})}^{\mu}\left(\Omega \times \mathbb{R}^{q} ; E, \tilde{E}\right)_{\kappa, \tilde{\kappa}}=S_{(\mathrm{cl})}^{\mu+\nu-\tilde{\nu}}\left(\Omega \times \mathbb{R}^{q} ; E, \tilde{E}\right)_{\kappa^{\prime}, \tilde{\kappa}^{\prime}} .
$$

Remark 1.1.6. (i) We have $p(y, \eta) \in S^{\mu}\left(\Omega \times \mathbb{R}^{q} ; \mathcal{K}^{s, \gamma}\left(X^{\wedge}\right), \mathcal{K}^{s-\mu, \gamma-\mu}\left(X^{\wedge}\right)\right)$ for every $s, \gamma \in \mathbb{R}$;

(ii) the operator of restriction $\mathrm{r}_{\partial X}: C_{0}^{\infty}\left(X^{\wedge}\right) \longrightarrow C_{0}^{\infty}\left((\partial X)^{\wedge}\right)$ extends to a continuous operator $\mathrm{r}_{\partial X}: \mathcal{K}^{s, \gamma}\left(X^{\wedge}\right) \longrightarrow \mathcal{K}^{s-\frac{1}{2}, \gamma-\frac{1}{2}}\left((\partial X)^{\wedge}\right)$ for every $s>$ $\frac{1}{2}, \gamma \in \mathbb{R}$, and we have $\mathrm{r}_{\partial X} \in S_{\mathrm{cl}}^{\frac{1}{2}}\left(\mathbb{R}^{q} ; \mathcal{K}^{s, \gamma}\left(X^{\wedge}\right), \mathcal{K}^{s-\frac{1}{2}, \gamma-\frac{1}{2}}\left((\partial X)^{\wedge}\right)\right)$.

Similar notation is used when $E$ or $\tilde{E}$ are Fréchet spaces with group action, that means, for instance, for $\tilde{E}$, that $\tilde{E}=\lim _{j \in \mathbb{N}} \tilde{E}^{j}$ for a sequence of Hilbert spaces $\tilde{E}^{j}$ with continuous embeddings $\tilde{E}^{j+1} \hookrightarrow \tilde{E}^{j} \hookrightarrow \ldots \hookrightarrow \tilde{E}^{0}$, where $\tilde{E}^{0}$ is endowed with a group action $\left\{\tilde{\kappa}_{\delta}\right\}_{\delta \in \mathbb{R}_{+}}$that restricts to group actions on $\tilde{E}^{j}$ for every $j$. Then $S_{(\mathrm{cl})}^{\mu}\left(\Omega \times \mathbb{R}^{q} ; E, \tilde{E}\right)$ is simply defined as the projective limit over all $S_{(\mathrm{cl})}^{\mu}(\Omega \times$ $\left.\mathbb{R}^{q} ; E, \tilde{E}^{j}\right)$.

Example 1.1.7. Let $E=L^{2}\left(\mathbb{R}_{+}\right) \oplus \mathbb{C}^{g_{-}}, \tilde{E}=\mathcal{S}\left(\overline{\mathbb{R}}_{+}\right) \oplus \mathbb{C}^{g_{+}}$for certain $g_{ \pm} \in \mathbb{N}$, where $\mathcal{S}\left(\overline{\mathbb{R}}_{+}\right):=\left.\mathcal{S}(\mathbb{R})\right|_{\overline{\mathbb{R}}_{+}}$, and $\mathcal{S}\left(\overline{\mathbb{R}}_{+}\right)=\lim _{k \in \mathbb{N}} H^{k, k}\left(\mathbb{R}_{+}\right),\left(H^{0,0}\left(\mathbb{R}_{+}\right)=L^{2}\left(\mathbb{R}^{+}\right)\right)$. Then, for the group action $\left\{\kappa_{\delta}\right\}_{\delta \in \mathbb{R}_{+}}, \kappa_{\delta} u(t):=\delta^{\frac{1}{2}} u(\delta t)$, on both $L^{2}\left(\mathbb{R}_{+}\right)$and $\mathcal{S}\left(\overline{\mathbb{R}}_{+}\right)$, and the trivial group action on $\mathbb{C}^{g_{ \pm}}$we can form the symbol spaces

$$
S_{\mathrm{cl}}^{\mu}\left(\Omega \times \mathbb{R}^{q} ; L^{2}\left(\mathbb{R}_{+}\right) \oplus \mathbb{C}^{g_{-}}, \mathcal{S}\left(\overline{\mathbb{R}}_{+}\right) \oplus \mathbb{C}^{g_{+}}\right) .
$$

If $g(y, \eta)$ is such a symbol, where the pointwise adjoint $g^{*}(y, \eta)$ belongs to

$$
S_{\mathrm{cl}}^{\mu}\left(\Omega \times \mathbb{R}^{q} ; L^{2}\left(\mathbb{R}_{+}\right) \oplus \mathbb{C}^{g_{+}}, \mathcal{S}\left(\overline{\mathbb{R}}_{+}\right) \oplus \mathbb{C}^{g_{-}}\right),
$$

then $g(y, \eta)$ can be expressed in terms of a $2 \times 2$ block matrix of operator functions with $a(y, \eta)$-wise kernel representation,

$$
\begin{gathered}
g_{11}: u(t) \rightarrow \int_{0}^{\infty} f_{11}\left(t, t^{\prime}\right) u\left(t^{\prime}\right) d t^{\prime}, \\
g_{21}: u(t) \rightarrow\left(\int_{0}^{\infty} f_{21, l}\left(t^{\prime}\right) u\left(t^{\prime}\right) d t^{\prime}\right)_{l=1, \ldots, g_{+}} \in \mathbb{C}^{g_{+}},
\end{gathered}
$$




$$
g_{12}: \mathbb{C}^{g_{-}} \ni\left(c_{1}, \ldots, c_{g_{-}}\right) \rightarrow \sum_{m=1}^{g_{-}} c_{m} f_{12, m}(t)
$$

for functions $f_{11}\left(t, t^{\prime}\right) \in \mathcal{S}\left(\overline{\mathbb{R}}_{+} \times \overline{\mathbb{R}}_{+}\right)=\left.\mathcal{S}(\mathbb{R} \times \mathbb{R})\right|_{\overline{\mathbb{R}}_{+} \times \overline{\mathbb{R}}_{+}}, f_{21, l}, f_{12, m} \in \mathcal{S}\left(\overline{\mathbb{R}}_{+}\right)$for all $l, m$.

With symbols we associate operators in the usual way, namely,

$$
\mathrm{Op}(a) u(y)=\iint e^{i\left(y-y^{\prime}\right) \eta} a\left(y, y^{\prime}, \eta\right) u\left(y^{\prime}\right) d y^{\prime} d \eta .
$$

Here we admit symbols $a\left(y, y^{\prime}, \eta\right) \in S_{(\mathrm{cl})}^{\mu}\left(\Omega \times \Omega \times \mathbb{R}^{q} ; E, \tilde{E}\right.$ ) (in the sense of a straightforward generalisation of the above notation to $\left(y, y^{\prime}\right)$-depending symbols). The definition of (1.7), first for $u \in C_{0}^{\infty}(\Omega, E)$ is based on oscillatory integral arguments. This gives us a continuous operator

$$
\mathrm{Op}(a): C_{0}^{\infty}(\Omega, E) \longrightarrow C^{\infty}(\Omega, \tilde{E}) .
$$

For references below we want to formulate the continuity between abstract edge Sobolev spaces:

Proposition 1.1.8. Let $a\left(y, y^{\prime}, \eta\right) \in S_{(\mathrm{cl})}^{\mu}\left(\Omega \times \Omega \times \mathbb{R}^{q} ; E, \tilde{E}\right)_{\kappa, \tilde{\kappa}}$. Then (1.8) extends to continuous operators

$$
\mathrm{Op}(a): \mathcal{W}_{\text {comp }}^{s}(\Omega, E)_{\kappa} \longrightarrow \mathcal{W}_{\text {loc }}^{s-\mu}(\Omega, \tilde{E})_{\tilde{\kappa}}
$$

between corresponding 'comp' and 'loc' generalisations of abstract edge Sobolev spaces on an open set $\Omega \subseteq \mathbb{R}^{q}$, for all $s \in \mathbb{R}$.

Observe that when we interpret $a\left(y, y^{\prime}, \eta\right)$ as an element of $S_{(\mathrm{cl})}^{\mu+\nu-\tilde{\nu}}(\Omega \times$ $\left.\mathbb{R}^{q} ; E, \tilde{E}\right)_{\kappa^{\prime}, \tilde{\kappa}^{\prime}}$, (via $a\left(y, y^{\prime}\right)$-dependent analogue of the relation (1.6)) we obtain the continuity

$$
\operatorname{Op}(a): \mathcal{W}_{\text {comp }}^{s+\nu}(\Omega, E)_{\kappa^{\prime}} \longrightarrow \mathcal{W}_{\text {loc }}^{s-\mu+\tilde{\nu}}(\Omega, \tilde{E})_{\tilde{\kappa}^{\prime}}
$$

for every $s \in \mathbb{R}$.

Note, in particular, that (1.5) is equal to the operator $O p\left(r_{\partial X}\right)$ with $r_{\partial X}$ as in Remark 1.1.6 (ii).

More generally, boundary value problems for a differential operator (0.4) on a (stretched) wedge $X^{\wedge} \times \Omega$ can be formulated in the framework of operators along $\Omega$ with amplitude functions taking values in boundary value problems on the infinite model cone $X^{\wedge}$. Differential boundary conditions will be represented as vectors $T={ }^{\mathrm{t}}\left(T_{1}, \ldots, T_{N}\right)$ of trace operators

$$
T_{j}=\mathrm{r}_{\partial X \times \Omega} B_{j}
$$


for differential operators $B_{j}$ of edge-degenerate form

$$
B_{j}=r^{-\mu_{j}} \sum_{k+|\beta| \leqq \mu_{j}} b_{j, k \beta}(r, y)\left(-r \partial_{r}\right)^{k}\left(r D_{y}\right)^{\beta},
$$

with coefficients $b_{j, k \beta}(r, y) \in C^{\infty}\left(\overline{\mathbb{R}}_{+} \times \Omega\right.$, Diff $\left.{ }^{\mu_{j}-(k+|\beta|)}\left(U^{\wedge}\right)\right)$ for a collar neighbourhood $U$ of $\partial X$ in $X$. For convenience, we assume again that the coefficients are independent of $(r, y)$ for large $|r, y|$. The number $N$ of boundary conditions is known together with the given elliptic differential operator $A$; for instance, for $\mu=2 m$ and $n>2$ we always have $N=m$.

Remark 1.1.9. As a consequence of the considerations before we have

$$
\begin{aligned}
t_{j}(y, \eta) & :=\mathrm{r}_{(\partial X)^{\wedge}} r^{-\mu_{j}} \sum_{k+|\beta| \leqq \mu_{j}} b_{j, k \beta}(r, y)\left(-r \partial_{r}\right)^{k}(r \eta)^{\beta} \\
& \in S^{\mu_{j}+\frac{1}{2}}\left(\Omega \times \mathbb{R}^{q} ; \mathcal{K}^{s, \gamma}\left(X^{\wedge}\right), \mathcal{K}^{s-\mu_{j}-\frac{1}{2}, \gamma-\mu_{j}-\frac{1}{2}}\left((\partial X)^{\wedge}\right)\right)
\end{aligned}
$$

for all $s>\mu_{j}+\frac{1}{2}, \gamma \in \mathbb{R}$.

We then obtain $T_{j}=\mathrm{Op}\left(t_{j}\right), j=1, \ldots, N$ or $T=\mathrm{Op}(t)$ for $t(y, \eta):=$ ${ }^{\mathrm{t}}\left(t_{1}(y, \eta), \ldots, t_{N}(y, \eta)\right)$. Thus, a boundary value problem

$$
A u=f, T u=g
$$

on $X^{\wedge} \times \Omega$ for an edge-degenerate operator $A$ gives rise to a continuous operator

$$
\begin{gathered}
\mathcal{A}:=\left(\begin{array}{c}
A \\
T
\end{array}\right): \mathcal{W}^{s, \gamma}\left(X^{\wedge} \times \mathbb{R}^{q}\right) \longrightarrow \mathcal{W}^{s-\mu, \gamma-\mu}\left(X^{\wedge} \times \mathbb{R}^{q}\right), \\
\mathcal{W}^{s-\mu, \gamma-\mu}\left(X^{\wedge} \times \mathbb{R}^{q}\right):=\mathcal{W}^{s-\mu, \gamma-\mu}\left(X^{\wedge} \times \mathbb{R}^{q}\right) \oplus \bigoplus_{j=1}^{N} \mathcal{W}^{s-\mu_{j}-\frac{1}{2}, \gamma-\mu_{j}-\frac{1}{2}}\left((\partial X)^{\wedge} \times \mathbb{R}^{q}\right),
\end{gathered}
$$

$s>\mu_{j}+\frac{1}{2}, j=1, \ldots, N, \gamma \in \mathbb{R}$.

Analogously, let $W$ be a compact manifold with edge $Y$ and boundary $V$ and $\mathbb{W}, \mathbb{V}$ be the associated stretched manifolds. Then we consider boundary value problems on $\mathbb{W}$, realised as continuous operators

$$
\mathcal{A}:=\left(\begin{array}{c}
A \\
T
\end{array}\right): \mathcal{W}^{s, \gamma}(\mathbb{W}) \longrightarrow \mathcal{W}^{s-\mu, \gamma-\mu}(\mathbb{W})
$$

for

$$
\mathcal{W}^{s-\mu, \gamma-\mu}(\mathbb{W}):=\mathcal{W}^{s-\mu, \gamma-\mu}(\mathbb{W}) \oplus \bigoplus_{j=1}^{N} \mathcal{W}^{s-\mu_{j}-\frac{1}{2}, \gamma-\mu_{j}-\frac{1}{2}}(\mathbb{V})
$$

between corresponding weighted Sobolev spaces on $\mathbb{W}$, for a differential operator $A$ on $\mathbb{W}_{\text {reg }}$ of order $\mu$ which is edge-degenerate locally near $Y$ in the splitting of 
variables $(r, x, y) \in X^{\wedge} \times \Omega$ and vectors of trace operators defined in terms of differential operators on $\mathbb{W}_{\text {reg }}$ of order $\mu_{j}$, edge-degenerate near $Y$, composed with the restriction operator to $\partial \mathbb{W}_{\text {reg }}$.

The global weighted Sobolev spaces $\mathcal{W}^{s, \gamma}(\mathbb{W})$ for the case of a stretched manifold with edge without boundary are defined as those subspaces of $H_{\mathrm{loc}}^{s}\left(\mathbb{W}_{\text {reg }}\right)$ that are locally near $Y$ in the variables $(r, x, y) \in X^{\wedge} \times \mathbb{R}^{q}$ of the form $\mathcal{W}^{s}\left(\mathbb{R}^{q}, \mathcal{K}^{s, \gamma}\left(X^{\wedge}\right)\right)$. In the case of $\mathbb{W}$ with boundary we set $\mathcal{W}^{s, \gamma}(\mathbb{W})=\left\{\left.u\right|_{\text {int } \mathbb{W}_{\text {reg }}}: u \in \mathcal{W}^{s, \gamma}(\mathbb{M})\right\}$ for the double $\mathbb{M}$ of $\mathbb{W}$ as explained before. Here, for simplicity, we assume that the boundary $\partial \mathbb{W}$ of our stretched manifold is a trivial $X$-bundle over $Y$ and that the transition maps between local 'singular charts' to $X^{\wedge} \times \mathbb{R}^{q}$ are independent of the axial variable $r \in \mathbb{R}_{+}$for small $r$ (these assumptions are not essential, cf. [4] for the case without boundary and Remark... below).

We will also employ spaces of the form

$$
\mathcal{W}^{s, \gamma}(\mathbb{W} ; G):=\mathcal{W}^{s, \gamma}(\mathbb{W}) \oplus \mathcal{W}^{s-\frac{1}{2}, \gamma-\frac{1}{2}}(\mathbb{V}, G)
$$

for a (smooth complex) vector bundle $G$ on $\mathbb{V}$ (we could consider spaces of distributional sections in suitable bundles on $\mathbb{W}$ as well; the corresponding generalisation is straightforward); throughout this paper we content ourselves with trivial bundles on $\mathbb{W}$ of fibre dimension 1.

\subsection{Boundary value problems with parameters}

In this section we introduce some tools on parameter-dependent boundary value problems on a (not necessarily compact) smooth manifold $X$ with boundary $\partial X$. First, if $N=2 X$ is the double of $X$, we have the subspace $L_{\mathrm{cl}}^{\mu}\left(N ; \mathbb{R}^{l}\right)_{\mathrm{tr}}$ of all $\tilde{A}(\lambda) \in L_{\mathrm{cl}}^{\mu}\left(N ; \mathbb{R}^{l}\right), \lambda \in \mathbb{R}^{l}, \mu \in \mathbb{Z}$, which have the transmission property at $\partial X$, cf. Boutet de Monvel [2]. If $\mathrm{e}^{+}$denotes the operator of extension by zero from $\operatorname{int} X\left(=\operatorname{int} X_{+}\right)$to $N\left(\right.$ applied to $H_{\text {loc }}^{s}(\operatorname{int} X)$ for $\left.s>-\frac{1}{2}\right)$ and $\mathrm{r}^{+}$the restriction of distributions from $N$ to int $X$ we can form the operators

$$
A(\lambda):=\mathrm{r}^{+} \tilde{A}(\lambda) \mathrm{e}^{+}
$$

which belong to $L_{\mathrm{cl}}^{\mu}\left(\operatorname{int} X ; \mathbb{R}^{l}\right)$.

Write $L_{\mathrm{cl}}^{\mu}\left(\operatorname{int} X ; \mathbb{R}^{l}\right)_{\text {tr }}$ for the set of all $(1.15)$ when $\tilde{A}(\lambda)$ runs through $L_{\mathrm{cl}}^{\mu}\left(N ; \mathbb{R}^{l}\right)_{\mathrm{tr}}$. Set

$$
H_{\text {loc (comp) }}^{s}(X):=\left\{\mathrm{r}^{+} u: u \in H_{\text {loc (comp) }}^{s}(2 X)\right\} .
$$

It is well known that every $A \in L_{\mathrm{cl}}^{\mu}\left(X ; \mathbb{R}^{l}\right)_{\text {tr }}$ induces continuous operators

$$
A: H_{\text {comp }}^{s}(X) \longrightarrow H_{\text {loc }}^{s-\mu}(X)
$$

for every real $s>-\frac{1}{2}$.

More generally, we can talk about spaces $L_{\mathrm{cl}}^{\mu}\left(N ; E, F ; \mathbb{R}^{l}\right)$ of classical parameterdependent pseudo-differential operators acting between distributional sections of 
(smooth complex) vector bundles $E$ and $F$ over a $C^{\infty}$ manifold $N$. For $N=$ $2 X, \mu \in \mathbb{Z}$, we also have corresponding subspaces with the transmission property at $\partial X$. We then obtain families of continuous operators

$$
A(\lambda):=\mathrm{r}^{+} \tilde{A}(\lambda) \mathrm{e}^{+}: H_{\mathrm{comp}}^{s}(X, E) \longrightarrow H_{\mathrm{loc}}^{s-\mu}(X, F)
$$

between Sobolev spaces of distributional sections in the bundles.

The parameter-dependent Boutet de Monvel calculus of 'boundary value problems' on $X$ is defined as the union of spaces $\mathcal{B}^{\mu, d}\left(X ; \mathbb{R}^{l}\right)$ of $2 \times 2$ block matrices $\mathcal{A}(\lambda)$ of operators of order $\mu$ and type $d \in \mathbb{N}$, continuous as operators

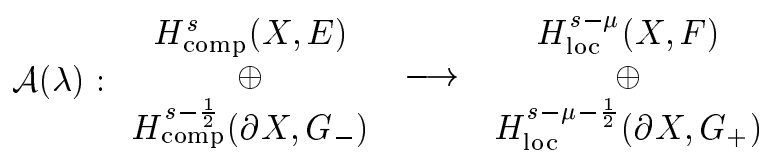

for $s>-\frac{1}{2}$, of the form

$$
\mathcal{A}(\lambda)=\operatorname{diag}(A(\lambda), 0)+\mathcal{G}(\lambda)+\mathcal{C}(\lambda)
$$

with arbitrary operators (1.16). Moreover, $\mathcal{G}(\lambda)$ are families of so called Green operators, and $\mathcal{C}(\lambda)$ are families of smoothing operators of order $\mu$ and type $d$, cf [2], [30], or [36]. Let us briefly recall the definition. Assume for convenience the bundles $E, F$ and $G_{ \pm}$to be trivial and of fibre dimension 1; the case of arbitrary bundles then is straightforward and omitted.

The space $\mathcal{B}^{-\infty, 0}(X)$ of smoothing operators (first for the case $l=0$ ) consists of $2 \times 2$ block matrix operators $\mathcal{C}=\left(\mathcal{C}_{i j}\right)_{i, j=1,2}$ where $\mathcal{C}_{11}, \mathcal{C}_{12}, \mathcal{C}_{21}$ and $\mathcal{C}_{22}$ are integral operators with kernels in $C^{\infty}(X \times X), C^{\infty}(X \times \partial X), C^{\infty}(\partial X \times X)$ and $C^{\infty}(\partial X \times \partial X)$, respectively. The space $\mathcal{B}^{-\infty, 0}(X)$ is Fréchet in a natural way, and we set $\mathcal{B}^{-\infty, 0}\left(X ; \mathbb{R}^{l}\right)=\mathcal{S}\left(\mathbb{R}^{l}, \mathcal{B}^{-\infty, 0}(X)\right)$. Now $\mathcal{B}^{-\infty, d}\left(X ; \mathbb{R}^{l}\right)$ for $d \in \mathbb{N}$ is the set of all operator families of the form

$$
\mathcal{C}(\lambda)=\mathcal{C}_{0}(\lambda)+\sum_{j=1}^{d} \mathcal{C}_{j}(\lambda) \operatorname{diag}\left(D^{j}, 0\right)
$$

for arbitrary $\mathcal{C}_{j}(\lambda) \in \mathcal{B}^{-\infty, 0}\left(X ; \mathbb{R}^{l}\right)$ and any differential operator $D$ on $X$ of first order that is equal to $\frac{\partial}{\partial x_{n}}$ in a collar neighbourhood $\cong \partial X \times[0,1)$ of the boundary, where $x_{n} \in[0,1)$ is the normal variable.

A Green operator of order $\mu$ and type 0 in the half-space $\Omega \times \mathbb{R}_{+} \ni\left(x^{\prime}, x_{n}\right), \Omega \subseteq$ $\mathbb{R}^{n-1}$ open, is defined (modulo smoothing operators) as

$$
\mathrm{Op}_{x^{\prime}}(g)(\lambda)
$$

for an operator-valued symbol $g\left(x^{\prime}, \xi^{\prime}, \lambda\right)$ where

$$
g\left(x^{\prime}, \xi^{\prime}, \lambda\right) \in S_{\mathrm{cl}}^{\mu}\left(\Omega \times \mathbb{R}^{n-1+l} ; L^{2}\left(\mathbb{R}_{+}\right) \oplus \mathbb{C}, \mathcal{S}\left(\overline{\mathbb{R}}_{+}\right) \oplus \mathbb{C}\right)_{\kappa, \kappa},
$$


cf. Example 1.1.7 for $g_{ \pm}=1$. The symbol space on the right of (1.20) is based on the group action $\kappa:=\left\{\operatorname{diag}\left(\kappa_{\delta}, \delta^{\frac{1}{2}}\right)\right\}_{\delta \in \mathbb{R}_{+}}$in the preimage and image spaces; we have also $g^{*}\left(x^{\prime}, \xi^{\prime}, \lambda\right) \in S_{\mathrm{cl}}^{\mu}\left(\Omega \times \mathbb{R}^{n-1+l} ; L^{2}\left(\mathbb{R}_{+}\right) \oplus \mathbb{C}, \mathcal{S}\left(\overline{\mathbb{R}}_{+}\right) \oplus \mathbb{C}\right)_{\boldsymbol{\kappa}^{*}, \boldsymbol{\kappa}^{*}}$ for $\boldsymbol{\kappa}^{*}:=\left\{\operatorname{diag}\left(\kappa_{\delta}, \delta^{-\frac{1}{2}}\right)\right\}_{\delta \in \mathbb{R}_{+}}$.

If $\chi: U \longrightarrow \Omega \times \overline{\mathbb{R}}_{+}$is a chart on $X$ near the boundary (for a coordinate neighbourhood $X$ with $U^{\prime}:=U \cap \partial X \neq \emptyset$, where $\chi^{\prime}:=\left.\chi\right|_{U^{\prime}}: U^{\prime} \longrightarrow \Omega$ defines a chart on $\partial X$ ) we can form the operator pull back of operators $\varphi \mathcal{G}(\lambda) \psi$ for localising functions $\varphi, \psi \in C_{0}^{\infty}\left(\Omega \times \overline{\mathbb{R}}_{+}\right)$to $X$.

Then $\mathcal{B}_{\mathrm{G}}^{\mu, 0}\left(X ; \mathbb{R}^{l}\right)$, the space of Green operators of order $\mu$ and type 0 on $X$, is (modulo $\mathcal{B}^{-\infty, 0}\left(X ; \mathbb{R}^{l}\right)$ ) defined to be the set of all locally finite sums of such operators $\left(\chi^{-1}\right)_{*}\{\varphi \mathrm{Op}(g)(\lambda) \psi\}$. Finally, $\mathcal{B}_{\mathrm{G}}^{\mu, d}\left(X ; \mathbb{R}^{l}\right)$, the space of all Green operators of order $\mu$ and type $d$, is the set of all $\mathcal{G}(\lambda)=\mathcal{G}_{0}(\lambda)+\sum_{j=1}^{d} \mathcal{G}_{j}(\lambda) \operatorname{diag}\left(D^{j}, 0\right)+\mathcal{C}(\lambda)$ for arbitrary $\mathcal{G}_{j}(\lambda) \in \mathcal{B}_{\mathrm{G}}^{\mu-j, 0}\left(X ; \mathbb{R}^{l}\right), \mathcal{C}(\lambda) \in \mathcal{B}^{-\infty, d}\left(X ; \mathbb{R}^{l}\right)$.

To complete the definition of $(1.17)$ we require $\mathcal{G}(\lambda)+\mathcal{C}(\lambda) \in \mathcal{B}_{\mathrm{G}}^{\mu, d}\left(X ; \mathbb{R}^{l}\right)$ (clearly, by notation, for global Green operators there is no need to split up a global smoothing operator $\mathcal{C}(\lambda)$ ).

We shall systematically employ the principal symbolic structure of boundary value problems; let us recall the definition for $\mathcal{B}^{\mu, d}(X)$ (which is the case $l=0$; the notions for arbitrary $l$ are analogous).

For simplicity, from now on we assume the vector bundles $E, F$ in the upper left corners to be trivial, while the bundles $G_{ \pm}$may be non-trivial.

The principal symbol of an $\mathcal{A} \in \mathcal{B}^{\mu, d}(X)$ is a pair

$$
\sigma(\mathcal{A}):=\left(\sigma_{\psi}(\mathcal{A}), \sigma_{\partial}(\mathcal{A})\right)
$$

where $\sigma_{\psi}(\mathcal{A}):=\sigma_{\psi}(A)$ is the standard homogeneous principal symbol of $A$ in the upper left corner as an element of $L_{\mathrm{cl}}^{\mu}(\operatorname{int} X)_{\mathrm{tr}}$ in the scalar case; then $\sigma_{\psi}(A) \in$ $C^{\infty}\left(T^{*} X \backslash 0\right)$ (with smoothness up to the boundary and usual homogeneity of order $\mu$ in the covariables). The boundary symbol $\sigma_{\partial}(\mathcal{A})$ parametrised by $\left(x^{\prime}, \xi^{\prime}\right) \in$ $T^{*}(\partial X) \backslash 0$ is defined by

$$
\sigma_{\partial}(\mathcal{A})\left(x^{\prime}, \xi^{\prime}\right)=\operatorname{diag}\left(\sigma_{\partial}(A)\left(x^{\prime}, \xi^{\prime}\right), 0\right)+\sigma_{\partial}(\mathcal{G})\left(x^{\prime}, \xi^{\prime}\right)
$$

where $\sigma_{\partial}(A)\left(x^{\prime}, \xi^{\prime}\right):=\mathrm{r}^{+} \sigma_{\psi}(A)\left(x^{\prime}, 0, \xi^{\prime}, D_{x_{n}}\right) \mathrm{e}^{+}$. We also write

$$
\mathrm{op}(p)\left(x^{\prime}, \xi^{\prime}\right) u\left(x_{n}\right)=\iint e^{i\left(x_{n}-\tilde{x}_{n}\right) \xi_{n}} p\left(x^{\prime}, x_{n}, \xi^{\prime}, \xi_{n}\right) u\left(\tilde{x}_{n}\right) d \tilde{x}_{n} d \xi_{n}
$$

and $\mathrm{op}^{+}(p)\left(x^{\prime}, \xi^{\prime}\right):=\mathrm{r}^{+} \mathrm{op}(p)\left(x^{\prime}, \xi^{\prime}\right) \mathrm{e}^{+}$for a symbol $p\left(x^{\prime}, x_{n}, \xi^{\prime}, \xi_{n}\right)$ in local coordinates $\left(x^{\prime}, x_{n}\right) \in \Omega \times \overline{\mathbb{R}}_{+}$near the boundary. If necessary, instead of op $(p)$ we write $\mathrm{op}_{x_{n}}(p)$.

Moreover, $\sigma_{\partial}(\mathcal{G})\left(x^{\prime}, \xi^{\prime}\right)$ in local coordinates on $\partial X$, i.e., $\left(x^{\prime}, \xi^{\prime}\right) \in T^{*} \Omega \backslash 0$, is nothing other than $\sigma_{\partial}(\mathcal{G})\left(x^{\prime}, \xi^{\prime}\right)=\sigma_{\partial}\left(\mathcal{G}_{0}\right)\left(x^{\prime}, \xi^{\prime}\right)+\sum_{j=1}^{d} \sigma_{\partial}\left(\mathcal{G}_{j}\right)\left(x^{\prime}, \xi^{\prime}\right) \frac{\partial^{j}}{\partial x_{n}^{j}}$ where 
$\sigma_{\partial}\left(\mathcal{G}_{j}\right)\left(x^{\prime}, \xi^{\prime}\right)=\left(g_{j}\right)_{(\mu-j)}\left(x^{\prime}, \xi^{\prime}\right)$ for $g$ replaced by the symbol $g_{j}$ in the local representation of $g_{j}$ as $\mathrm{Op}_{x^{\prime}}\left(g_{j}\right)$. Note that homogeneity of the boundary symbol means $\sigma_{\partial}(\mathcal{A})\left(x^{\prime}, \delta \xi^{\prime}\right)=\delta^{\mu} \boldsymbol{\kappa}_{\delta} \sigma_{\partial}(\mathcal{A})\left(x^{\prime}, \xi^{\prime}\right) \boldsymbol{\kappa}_{\delta}^{-1}$ for all $\delta \in \mathbb{R}_{+},\left(x^{\prime}, \xi^{\prime}\right) \in T^{*}(\partial X) \backslash 0$.

For our applications it is essential to admit $X$ to be a non-compact $C^{\infty}$ manifold with boundary. Similarly as in the case without boundary we have a notion of properly supported operators in $\mathcal{B}^{\mu, d}(X)$ (we do not repeat this here but tacitly use it). Then every $\mathcal{A} \in \mathcal{B}^{\mu, d}(X)$ has a decomposition as $\mathcal{A}=\mathcal{A}_{0}+\mathcal{C}$ for a properly supported $\mathcal{A}_{0} \in \mathcal{B}^{\mu, d}(X)$ and a $\mathcal{C} \in \mathcal{B}^{-\infty, d}(X)$. Properly supported operators preserve the 'comp' Sobolev spaces and may be extended as continuous map between the respective 'loc' Sobolev spaces.

Theorem 1.2.1. Let $\mathcal{A} \in \mathcal{B}^{\mu, d}(X), \tilde{\mathcal{A}} \in \mathcal{B}^{\tilde{\mu}, \tilde{d}}(X)$, and let $\mathcal{A}$ or $\tilde{\mathcal{A}}$ be properly supported. Then $\mathcal{A} \tilde{\mathcal{A}} \in \mathcal{B}^{\mu+\tilde{\mu}, h}(X)$ (provided that the bundles in the image of $\mathcal{A}$ fit to the one in the domain of $\tilde{\mathcal{A}})$, for $h=\max (d+\tilde{\mu}, \tilde{d})$, and we have $\sigma(\mathcal{A} \tilde{\mathcal{A}})=$ $\sigma(\mathcal{A}) \sigma(\tilde{\mathcal{A}})$ (with componentwise composition).

An $\mathcal{A} \in \mathcal{B}^{\mu, d}(X)$ is said to be elliptic if

(i) $\sigma_{\psi}(A) \neq 0$ on $T^{*} X \backslash 0$;

(ii) $\sigma_{\partial}(\mathcal{A})$ induces isomorphisms

$$
\sigma_{\partial}(\mathcal{A})\left(x^{\prime}, \xi^{\prime}\right): \begin{gathered}
H^{s}\left(\mathbb{R}_{+}\right) \\
G_{-, x^{\prime}}
\end{gathered} \longrightarrow \begin{gathered}
H^{s-\mu}\left(\mathbb{R}_{+}\right) \\
G_{+, x^{\prime}}
\end{gathered}
$$

for every $x^{\prime} \in \partial X$ (with subscript ' $x$ ' denoting the fibres over $x^{\prime}$ ).

Theorem 1.2.2. An elliptic operator $\mathcal{A} \in \mathcal{B}^{\mu, d}(X)$ has a (properly supported) parametrix $\mathcal{P} \in \mathcal{B}^{-\mu,(d-\mu)^{+}}(X)$ in the sense that $\mathcal{P} \mathcal{A}-\mathcal{I} \in \mathcal{B}^{-\infty, d_{l}}(X), \mathcal{A} \mathcal{P}-\mathcal{I} \in$ $\mathcal{B}^{-\infty, d_{r}}(X)$, (with $d_{l}=\max (\mu, d), d_{r}=(d-\mu)^{+}$, and $\mathcal{I}$ denoting the respective identity operators, $\nu^{+}:=\max (\nu, 0)$ for any $\left.\nu \in \mathbb{R}\right)$.

We now pass to the so called Mellin quantisation of families $\mathcal{A}(z, \eta) \in \mathcal{B}^{\mu, d}\left(X ; \Gamma_{\beta} \times \mathbb{R}^{q}\right)$; here $\Gamma_{\beta}:=\{z \in \mathbb{C}: \operatorname{Re} z=\beta\}$ for some real $\beta$, and $\Gamma_{\beta} \times \mathbb{R}^{q}$ in the notation of parameter-dependent families means that $(\operatorname{Im} z, \eta)$ is treated as a parameter when $z$ varies on $\Gamma_{\beta}$.

By $\mathcal{O}(U, E)$ for $U \subseteq \mathbb{C}$ open and a Fréchet space $E$ we denote the space of all $E$ valued functions that are holomorphic in $U$ (in the Fréchet topology of uniform convergence on compact sets).

Definition 1.2.3. Let $\mathcal{B}^{\mu, d}\left(X ; \mathbb{C} \times \mathbb{R}^{q}\right)$ denote the set of all $\mathcal{H}(z, \eta) \in$ $\mathcal{O}\left(\mathbb{C}, \mathcal{B}^{\mu, d}\left(X ; \mathbb{R}^{q}\right)\right)$ such that $\mathcal{H}(\beta+i \rho, \eta) \in \mathcal{B}^{\mu, d}\left(X ; \mathbb{R}_{\rho, \eta}^{1+q}\right)$ for every $\beta \in \mathbb{R}$, uniformly in compact intervals. 
The operator functions of the latter definition will be employed as amplitude functions of pseudo-differential operators based on the Mellin transform in $\mathbb{R}_{4} \ni$ $r, M u(z)=\int_{0}^{\infty} r^{z-1} u(r) d r$.

Given $\mathcal{H}\left(r, r^{\prime}, z\right) \in C^{\infty}\left(\mathbb{R}_{+} \times \mathbb{R}_{+} \times \Gamma_{\frac{1}{2}-\gamma}\right)$ for a weight $\gamma \in \mathbb{R}$ we set

$$
\mathrm{op}_{M}^{\gamma}(\mathcal{H}) u(r):=\iint\left(\frac{r}{r^{\prime}}\right)^{-\left(\frac{1}{2}-\gamma+i \rho\right)} \mathcal{H}\left(r, r^{\prime}, \frac{1}{2}-\gamma+i \rho\right) u\left(r^{\prime}\right) \frac{d r^{\prime}}{r^{\prime}} d \rho,
$$

first for $u$ of compact support in $r \in \mathbb{R}_{+}$, and then for $u$ in more general weighted distributions spaces (also vector-valued ones).

Let $\Omega \subseteq \mathbb{R}^{p}$ be any open set and fix $\beta \in \mathbb{R}$.

Theorem 1.2.4. There is a continuous map

$$
C^{\infty}\left(\overline{\mathbb{R}}_{+} \times \Omega, \mathcal{B}^{\mu, d}\left(X ; \mathbb{R}_{\tilde{\rho}, \tilde{\eta}}^{1+q}\right)\right) \longrightarrow C^{\infty}\left(\overline{\mathbb{R}}_{+} \times \Omega, \mathcal{B}^{\mu, d}\left(X ; \mathbb{C} \times \mathbb{R}_{\tilde{\eta}}^{q}\right)\right),
$$

called a Mellin quantisation $\tilde{\mathcal{A}}(r, y, \tilde{\rho}, \tilde{\eta}) \longrightarrow \tilde{\mathcal{H}}(r, y, z, \tilde{\eta})$, such that, when we form $\mathcal{A}(r, y, \rho, \eta):=\tilde{\mathcal{A}}(r, y, r \rho, r \eta), \mathcal{H}(r, y, z, \eta):=\tilde{\mathcal{H}}(r, y, z, r \eta)$ we have

$$
\mathrm{op}_{r}(\mathcal{A})(y, \eta)=\mathrm{op}_{M}^{\gamma}(\mathcal{H})(y, \eta),
$$

$\bmod C^{\infty}\left(\Omega, \mathcal{B}^{-\infty, d}\left(\mathbb{R}_{+} \times X ; \mathbb{R}^{q}\right)\right)$, for every $\gamma \in \mathbb{R}$. Moreover, setting $\mathcal{A}_{0}(r, y, \rho, \eta):=\tilde{\mathcal{A}}(0, y, r \rho, r \eta), \mathcal{H}_{0}(r, y, z, \eta):=\tilde{\mathcal{H}}(0, y, z, r \eta)$ we also have

$$
\operatorname{op}_{r}\left(\mathcal{A}_{0}\right)(y, \eta)=\operatorname{op}_{M}^{\gamma}\left(\mathcal{H}_{0}\right)(y, \eta)
$$

$\bmod C^{\infty}\left(\Omega, \mathcal{B}^{-\infty, d}\left(\mathbb{R}_{+} \times X ; \mathbb{R}^{q}\right)\right)$, for every $\gamma \in \mathbb{R}$.

Concerning proofs of the results of this section, see, e.g., [13].

\subsection{Edge quantisation}

By edge quantisation we understand a (non-canonical) map from the space of edge-degenerate (pseudo-differential) boundary value problems into the so called edge algebra of operators, acting between weighted edge Sobolev spaces (and subspaces with asymptotics). An edge-degenerate boundary value problem on an (open stretched) wedge $X^{\wedge} \times \Omega \ni(r, x, y)$ is an operator of the form

$$
\mathcal{A}\left(r, y, r D_{r}, r D_{y}\right):=\mathrm{Op}_{y} \mathrm{op}_{r}(\mathcal{A}),
$$

for an operator-valued amplitude function $\mathcal{A}(r, y, \rho, \eta)=\tilde{\mathcal{A}}(r, y, r \rho, r \eta)$ where

$$
\tilde{\mathcal{A}}(r, y, \tilde{\rho}, \tilde{\eta}) \in C^{\infty}\left(\overline{\mathbb{R}}_{+} \times \Omega, \mathcal{B}^{\mu, d}\left(X ; \mathbb{R}_{\tilde{\rho}, \tilde{\eta}}^{1+q}\right)\right) .
$$

In order to construct the edge-quantised operators we first fix cut-off functions $\omega(r), \omega_{0}(r), \omega_{1}(r)$ such that $\omega_{0} \equiv 1$ on $\operatorname{supp} \omega, \omega \equiv 1$ on $\operatorname{supp} \omega_{1}$, and arbitrary 
cut-off functions $\sigma(r)$ and $\tilde{\sigma}(r)$, and form the operator-valued amplitude functions of the form

$$
\begin{aligned}
a(y, \eta) & :=\sigma(r)\left\{\omega(r[\eta]) r^{-\mu} \operatorname{op}_{M}^{\gamma-\frac{n}{2}}(\mathcal{H})(y, \eta) \omega_{0}(r[\eta])\right. \\
& \left.+(1-\omega(r[\eta])) r^{-\mu} \operatorname{op}_{r}(\mathcal{A})(y, \eta)\left(1-\omega_{1}(r[\eta])\right)\right\} \tilde{\sigma}(r)
\end{aligned}
$$

where $\mathcal{A}$ is given as before, and $\mathcal{H}$ associated with $\mathcal{A}$ via Theorem 1.2.4. Observe that

$$
a(y, \eta)=\sigma(r) \operatorname{op}_{r}(\mathcal{A})(y, \eta) \tilde{\sigma}(r)
$$

modulo an element of $C^{\infty}\left(\Omega, \mathcal{B}^{-\infty, d}\left(X^{\wedge} ; \mathbb{R}_{\eta}^{q}\right)\right)$, and $a(y, \eta) \in C^{\infty}\left(\Omega, \mathcal{B}^{\mu, d}\left(X^{\wedge} ; \mathbb{R}^{q}\right)\right)$. In addition we know that $a(y, \eta) \in S^{\mu}\left(\Omega \times \mathbb{R}^{q} ; E, \tilde{E}\right)$ for $E:=\mathcal{K}^{s, \gamma}\left(X^{\wedge}\right) \oplus$ $\mathcal{K}^{s-\frac{1}{2}, \gamma-\frac{1}{2}}\left((\partial X)^{\wedge}, G_{-}\right), \tilde{E}:=\mathcal{K}^{s-\mu, \gamma-\mu}\left(X^{\wedge}\right) \oplus \mathcal{K}^{s-\mu-\frac{1}{2}, \gamma-\mu-\frac{1}{2}}\left((\partial X)^{\wedge}, G_{+}\right)$where the group actions in $E$ or $\tilde{E}$ are given as

$$
\kappa_{\delta}\left(u(r, x) \oplus v\left(r, x^{\prime}\right)\right):=\delta^{\frac{n+1}{2}}\left(u(\delta r, x) \oplus v\left(\delta r, x^{\prime}\right)\right),
$$

$\delta \in \mathbb{R}_{+}$. In these relations the smoothness $s$ is arbitrary, up to the condition $s>d-\frac{1}{2}$.

With (1.23) we associate the homogeneous principal edge symbol

$$
\begin{aligned}
\sigma_{\wedge}(a)(y, \eta) & :=\omega(r|\eta|) r^{-\mu} \operatorname{op}_{M}^{\gamma-\frac{n}{2}}\left(\mathcal{H}_{0}\right)(y, \eta) \omega_{0}(r|\eta|) \\
& +(1-\omega(r|\eta|)) r^{-\mu} \operatorname{op}_{r}\left(\mathcal{A}_{0}\right)(y, \eta)\left(1-\omega_{1}(r|\eta|)\right)
\end{aligned}
$$

with $\mathcal{H}_{0}$ and $\mathcal{A}_{0}$ as in Theorem 1.2.4.

In parametrix constructions for elliptic operators we shall have to consider compositions of operator-valued amplitude functions of the kind (1.23), for different orders $\mu$. It is known, cf. [33], [13], that such compositions are again of that structure, modulo so called smoothing Mellin plus Green symbols. In our calculus below we need a few details; therefore we recall the main notation.

Let $\mathcal{M}^{-\infty, d}\left(X ; \Gamma_{\beta}\right), \beta \in \mathbb{R}$, denote the space of all

$$
f(z) \in \mathcal{O}\left(\beta-\varepsilon<\operatorname{Re} z<\beta+\varepsilon, \mathcal{B}^{-\infty, d}(X)\right)
$$

for some $\varepsilon(f)>0$, such that

$$
f(\vartheta+i \rho) \in \mathcal{S}\left(\mathbb{R}_{\rho}, \mathcal{B}^{-\infty, d}(X)\right)
$$

for every $\beta-\varepsilon<\vartheta<\beta+\varepsilon$, uniformly in compact subintervals.

For every $f(y, z) \in C^{\infty}\left(\Omega, \mathcal{M}^{-\infty, d}\left(X ; \Gamma_{\frac{n+1}{2}-\gamma}\right)\right)$ we form the operators

$$
m(y, \eta):=r^{-\mu} \omega(r[\eta]) \operatorname{op}_{M}^{\gamma-\frac{n}{2}}(f)(y) \tilde{\omega}(r[\eta])
$$

for any fixed cut-off functions $\omega, \tilde{\omega}$. We then have

$$
m(y, \eta) \in S_{\mathrm{cl}}^{\mu}\left(\Omega \times \mathbb{R}^{q} ; E, E^{\infty}\right)
$$


for $E^{\infty}:=\mathcal{K}^{\infty, \gamma}\left(X^{\wedge}\right) \oplus \mathcal{K}^{\infty, \gamma-\frac{1}{2}}\left((\partial X)^{\wedge}, G_{-}\right)$, and $E$ as before, for arbitrary $s>$ $d-\frac{1}{2}$, and the group action (1.25) in $E$ and $E^{\infty}$, respectively. In this notation we keep in mind the bundles $G_{ \pm}$that are given in connection with $f(y, z)$. The operator family (1.26) is called a smoothing Mellin symbol of the edge calculus of boundary value problems. The homogeneous principal edge symbol of (1.26) is defined as

$$
\sigma_{\wedge}(m)(y, \eta):=r^{-\mu} \omega(r|\eta|) \operatorname{op}_{M}^{\gamma-\frac{n}{2}}(f)(y) \tilde{\omega}(r|\eta|)
$$

To complete the symbolic structure of such problems it remains to define the Green symbols. Those will include (by notation) also additional edge data of trace and potential type with respect to the edge. That means we are talking about $3 \times 3$ block matrix-valued symbols. Let us set

$$
\begin{gathered}
\mathcal{S}^{\gamma}\left(X^{\wedge}\right):=\omega \mathcal{K}^{\infty, \gamma}\left(X^{\wedge}\right)+(1-\omega) \mathcal{S}\left(\overline{\mathbb{R}}_{+}, C^{\infty}(X)\right) \\
\mathcal{S}^{\gamma}\left((\partial X)^{\wedge}, G\right):=\omega \mathcal{K}^{\infty, \gamma}\left((\partial X)^{\wedge}, G\right)+(1-\omega) \mathcal{S}\left(\overline{\mathbb{R}}_{+}, C^{\infty}(X, G)\right)
\end{gathered}
$$

for some cut-off functions $\omega(r)$, and set

$$
\mathcal{S}^{\gamma}\left(X^{\wedge} ; G\right):=\mathcal{S}^{\gamma}\left(X^{\wedge}\right) \oplus \mathcal{S}^{\gamma-\frac{1}{2}}\left((\partial X)^{\wedge}, G\right) .
$$

Similarly, we also set

$$
\mathcal{K}^{s, \gamma}\left(X^{\wedge} ; G\right):=\mathcal{K}^{s, \gamma}\left(X^{\wedge}\right) \oplus \mathcal{K}^{s-\frac{1}{2}, \gamma-\frac{1}{2}}\left((\partial X)^{\wedge}, G\right) .
$$

In spaces of the kind $\mathcal{S}^{\gamma}\left(X^{\wedge} ; G\right) \oplus \mathbb{C}^{j}$ or $\mathcal{K}^{s, \gamma}\left(X^{\wedge} ; G\right) \oplus \mathbb{C}^{j}$ we consider the group action $\kappa:=\left\{\kappa_{\delta}\right\}_{\delta \in \mathbb{R}_{+}}$, defined by

$$
\kappa_{\delta}\left(u(r, x) \oplus v\left(r, x^{\prime}\right) \oplus c\right):=\delta^{\frac{n+1}{2}}\left(u(\delta r, x) \oplus v\left(\delta r, x^{\prime}\right)\right) \oplus \delta c
$$

$\delta \in \mathbb{R}_{+}$. The choice of the group action (1.28) will be useful for having transparent order conventions in our operator-valued symbols. There are also the natural group actions on the spaces $\mathcal{K}^{s, \gamma}\left(X^{\wedge} ; G\right) \oplus \mathbb{C}^{j}$ given by

$$
\left.u(r, x) \oplus v\left(r, x^{\prime}\right) \oplus c \rightarrow \delta^{\frac{n+1}{2}} u(\delta r, x) \oplus \delta^{\frac{n}{2}} v\left(\delta r, x^{\prime}\right)\right) \oplus c,
$$

$\delta \in \mathbb{R}_{+}$. According to Remark 1.1.2 we then have the natural identification

$$
\begin{aligned}
\mathcal{W}^{s}\left(\mathbb{R}^{q}, \mathcal{K}^{s, \gamma}\left(X^{\wedge}\right) \oplus \mathcal{K}^{s-\frac{1}{2}, \gamma-\frac{1}{2}}\left((\partial X)^{\wedge}\right) \oplus \mathbb{C}^{j}\right)_{\kappa}= \\
\mathcal{W}^{s}\left(\mathbb{R}^{q}, \mathcal{K}^{s, \gamma}\left(X^{\wedge}\right)\right) \oplus \mathcal{W}^{s-\frac{1}{2}}\left(\mathbb{R}^{q}, \mathcal{K}^{s-\frac{1}{2}, \gamma-\frac{1}{2}}\left((\partial X)^{\wedge}\right)\right) \oplus H^{s-1}\left(\mathbb{R}^{q}, \mathbb{C}^{j}\right)
\end{aligned}
$$

A Green symbol $\mathfrak{g}(y, \eta)$ of order $\mu \in \mathbb{R}$ and type 0 is defined by

$$
\mathfrak{g}(y, \eta) \in S_{\mathrm{cl}}^{\mu}\left(\Omega \times \mathbb{R}^{q} ; \mathcal{K}^{s, \gamma}\left(X^{\wedge} ; G_{-}\right) \oplus \mathbb{C}^{j_{-}}, \mathcal{S}^{\gamma-\mu+\varepsilon}\left(X^{\wedge} ; G_{+}\right) \oplus \mathbb{C}^{j_{+}}\right)_{\boldsymbol{\kappa}, \boldsymbol{\kappa}}
$$

for an $\varepsilon(\mathfrak{g})>0$ and all $s>-\frac{1}{2}$, and a similar condition for the pointwise adjoint based on group actions $\kappa^{*}$, similarly as in the definition of Green symbols for 
boundary symbols. Then a Green symbol of order $\mu \in \mathbb{R}$ and type $d \in \mathbb{N}$ is defined by

$$
\mathfrak{g}(y, \eta)=\mathfrak{g}_{0}(y, \eta)+\sum_{j=1}^{d} \mathfrak{g}_{j}(y, \eta) \operatorname{diag}\left(D^{j}, 0,0\right)
$$

for Green symbols $\mathfrak{g}_{j}(y, \eta)$ of order $\mu$ and type $0, j=0, \ldots, d$, with $D$ being of analogous meaning as in (1.18) (i.e., differentiation of first order transversal to $\partial X)$.

The homogeneous principal edge symbol of (1.30) is defined as

$$
\sigma_{\wedge}(\mathfrak{g})(y, \eta)=\sigma_{\wedge}\left(\mathfrak{g}_{0}\right)(y, \eta)+\sum_{j=1}^{d} \sigma_{\wedge}\left(\mathfrak{g}_{j}\right)(y, \eta) \operatorname{diag}\left(D^{j}, 0,0\right)
$$

where $\sigma_{\wedge}\left(\mathfrak{g}_{j}\right)(y, \eta)$ is the homogeneous principal symbol of $\mathfrak{g}_{j}(y, \eta)$ as an element of the space on the right of (1.29). Observe that when we set

$$
\mathfrak{a}(y, \eta):=\left(\begin{array}{cc}
a(y, \eta)+m(y, \eta) & 0 \\
0 & 0
\end{array}\right)+\mathfrak{g}(y, \eta)
$$

we have the homogeneity $\mathfrak{a}(y, \delta \eta)=\delta^{\mu} \boldsymbol{\kappa}_{\delta} \mathfrak{a}(y, \eta) \boldsymbol{\kappa}_{\delta}^{-1}$ for all $\delta \in \mathbb{R}_{+},(y, \eta) \in T^{*} \Omega \backslash 0$, where $\left\{\boldsymbol{\kappa}_{\delta}\right\}_{\delta \in \mathbb{R}_{+}}$is the group action in (1.28) (of course, defined for the involved dimensions $j_{ \pm}$in the third component).

\subsection{Edge boundary value problems}

Edge boundary value problems may be introduced in terms of operator block matrices acting between spaces of the kind $\mathcal{W}^{s, \gamma}(\mathbb{W} ; G) \oplus H^{s-1}(Y, J)$ for suitable bundles $G$ on $\mathbb{V}$ and $J$ on $Y$, respectively (cf. the notation (1.14)). Recall that the spaces $\mathcal{W}^{s, \gamma}(\mathbb{W} ; G)$ are defined with respect to the natural group actions in $\mathcal{K}^{s, \gamma}\left(X^{\wedge}\right)$ and $\mathcal{K}^{s-\frac{1}{2}, \gamma-\frac{1}{2}}\left((\partial X)^{\wedge}\right)$-spaces, related to the dimensions.

Let us first define the global smoothing operators of the calculus. A smoothing operator $\mathfrak{C}$ of type 0 is characterised by the condition of continuity

$$
\mathfrak{C}: \begin{gathered}
\mathcal{W}^{s, \gamma}\left(\mathbb{W} ; G_{-}\right) \\
H^{s^{\prime}}\left(Y, J_{-}\right)
\end{gathered} \longrightarrow \begin{gathered}
\mathcal{W}^{\infty, \gamma-\mu+\varepsilon}\left(\mathbb{W} ; G_{+}\right) \\
\longrightarrow
\end{gathered}
$$

for all $s>-\frac{1}{2}, s^{\prime} \in \mathbb{R}$, and some $\varepsilon(\mathfrak{C})>0$ together with a similar property of the formal adjoint; here $G_{ \pm}$and $J_{ \pm}$are bundles on $\mathbb{V}$ and $Y$, respectively. Let $\mathfrak{Y}^{-\infty, 0}(\mathbb{W})$ denote the space of all those operators (if necessary, we also write $\mathfrak{Y}^{-\infty, 0}(\mathbb{W} ; \boldsymbol{v})$ for $\left.\boldsymbol{v}:=\left(G_{-}, G_{+} ; J_{-}, J_{+}\right)\right)$.

A smoothing operator $\mathfrak{C}$ of type $d \in \mathbb{N}$ is a sum $\mathfrak{C}:=\mathfrak{C}_{0}+\sum_{j=1}^{d} \mathfrak{C}_{j} \operatorname{diag}\left(D^{j}, 0,0\right)$ for arbitrary elements $\mathfrak{C}_{j} \in \mathfrak{Y}^{-\infty, 0}(\mathbb{W}), j=0, \ldots, d$, and a first order differential operator $D$ on $\mathbb{W}$ that is equal to the differentiation in normal direction to $\partial \mathbb{W}_{\text {reg }}$ in a collar neighbourhood of the boundary. 
Definition 1.4.1. Given a compact manifold $W$ with edge $Y$, by an edge boundary value problem of order $\mu \in \mathbb{Z}$ and type $d \in \mathbb{N}$ we understand a $3 \times 3$ - block matrix operator $\mathfrak{A}$ that is modulo $\mathfrak{Y}^{-\infty, d}(\mathbb{W})$ of the following form: Writing $\mathfrak{A}=$ $\left(\mathfrak{A}_{i j}\right)_{i, j=1,2,3}$ we require

(i) $\left(\mathfrak{A}_{i j}\right)_{i, j=1,2} \in \mathcal{B}^{\mu, d}\left(\mathbb{W}_{\text {reg }}\right)$,

(ii) locally near $\mathbb{W}_{\text {sing }}$ in the splitting of variables $(r, x, y) \in X^{\wedge} \times \Omega$ we have $\mathfrak{A}=\mathrm{Op}_{y}(\mathfrak{a})$ for an amplitude function (1.31) for symbols of the form (1.23) and (1.26) and a Green symbol (1.30).

By $\mathfrak{Y}^{\mu, d}(\mathbb{W})$ we denote the space of all edge boundary value problems on $\mathbb{W}$, of order $\mu \in \mathbb{Z}$ and type $d \in \mathbb{N}$; if necessary, we also write $\mathfrak{Y}^{\mu, d}(\mathbb{W} ; \boldsymbol{v})$ when we refer to the bundle data $\boldsymbol{v}:=\left(G_{-}, G_{+} ; J_{-}, J_{+}\right)$.

Theorem 1.4.2. Every $\mathfrak{A} \in \mathfrak{Y}^{\mu, d}(\mathbb{W} ; \boldsymbol{v})$ induces a continuous operator

$$
\mathfrak{A}: \begin{gathered}
\mathcal{W}^{s, \gamma}\left(\mathbb{W} ; G_{-}\right) \\
H^{s-1}\left(Y, J_{-}\right)
\end{gathered} \longrightarrow \begin{gathered}
\mathcal{W}^{s-\mu, \gamma-\mu}\left(\mathbb{W} ; G_{+}\right) \\
H^{s-\mu-1}\left(Y, J_{+}\right)
\end{gathered}
$$

for every real $s>d-\frac{1}{2}$.

We now define the principal symbolic structure of operators $\mathfrak{A} \in \mathfrak{Y}^{\mu, d}(\mathbb{W})$.

Definition 1.4.3. Given $\mathfrak{A} \in \mathfrak{Y}^{\mu, d}(\mathbb{W} ; \boldsymbol{v})$, the principal symbolic hierarchy

$$
\sigma(\mathfrak{A}):=\left(\sigma_{\psi}(\mathfrak{A}), \sigma_{\partial}(\mathfrak{A}), \sigma_{\wedge}(\mathfrak{A})\right)
$$

consists of

(i) $\sigma_{\psi}(\mathfrak{A})$, the interior symbol, defined by

$$
\sigma_{\psi}(\mathfrak{A}):=\sigma_{\psi}\left(\left(\mathfrak{A}_{i j}\right)_{i, j=1,2}\right)
$$

in the sense of the interior symbolic structure of $\mathcal{B}^{\mu, d}\left(\mathbb{W}_{\text {reg }}\right), \quad c f$. Definition 1.4.1(i);

(ii) $\sigma_{\partial}(\mathfrak{A})$, the boundary symbol, defined by

$$
\sigma_{\partial}(\mathfrak{A}):=\sigma_{\partial}\left(\left(\mathfrak{A}_{i j}\right)_{i, j=1,2}\right)
$$

in the sense of the boundary symbolic structure of $\mathcal{B}^{\mu, d}\left(\mathbb{W}_{\text {reg }}\right), \quad c f$. Definition 1.4.1(i); 
(iii) $\sigma_{\wedge}(\mathfrak{A})$, the edge symbol, defined as the operator family

$$
\sigma_{\wedge}(\mathfrak{A})(y, \eta): \begin{gathered}
\mathcal{K}^{s, \gamma}\left(X^{\wedge} ; G_{-}\right) \\
\oplus \\
J_{-, y}
\end{gathered} \rightarrow \begin{gathered}
\mathcal{K}^{s-\mu, \gamma-\mu}\left(X^{\wedge} ; G_{+}\right) \\
J_{+, y}
\end{gathered}
$$

for $(y, \eta) \in T^{*} Y \backslash 0$ for any $s>d-\frac{1}{2}$.

Observe that the $2 \times 2$ upper left corner $\sigma_{\wedge}\left(\left(\mathfrak{A}_{i j}\right)_{i, j=1,2}\right)(y, \eta)$ for every fixed $(y, \eta) \in T^{*} Y \backslash 0$ is a family of elliptic boundary value problems on the infinite (stretched) cone $X^{\wedge}$ with boundary $(\partial X)^{\wedge}$, with ellipticity referring to the principal symbolic hierarchy of the cone calculus (Fuchs type symbols, conormal symbols, exit symbols), cf. [31], [32] and [13]. In particular, the principal conormal symbol is a family of maps

$$
\sigma_{M} \sigma_{\wedge}(\mathfrak{A})(y, z): \boldsymbol{H}^{s}\left(X ; G_{-}\right) \longrightarrow \boldsymbol{H}^{s-\mu}\left(X ; G_{+}\right)
$$

$s \in \mathbb{R}$ sufficiently large, where we set $\boldsymbol{H}^{s}(X ; G):=H^{s}(X) \oplus H^{s-\frac{1}{2}}(\partial X, G)$. Note that (1.34) only depends on the $2 \times 2$-upper left corner $\mathcal{A}$ of $\mathfrak{A}$; so we also set $\sigma_{M} \sigma_{\wedge}(\mathfrak{A})=\sigma_{M} \sigma_{\wedge}(\mathcal{A})$.

Remark 1.4.4. By virtue of the edge-degeneracy of our operators the components $\sigma_{\psi}(\mathfrak{A})$ and $\sigma_{\partial}(\mathfrak{A})$ in the local splittings of variables $(r, x, y) \in X^{\wedge} \times \Omega$ and $\left(r, x^{\prime}, y\right) \in(\partial X)^{\wedge} \times \Omega$ with the covariables $(\rho, \xi, \eta)$ and $\left(\rho, \xi^{\prime}, \eta\right)$, respectively, have the following specific form:

$$
\begin{gathered}
\sigma_{\psi}(\mathfrak{A})(r, x, y, \rho, \xi, \eta)=r^{-\mu} \tilde{\sigma}_{\psi}(\mathfrak{A})(r, x, y, r \rho, \xi, r \eta) \text { and } \\
\sigma_{\partial}(\mathfrak{A})\left(r, x^{\prime}, y, \rho, \xi^{\prime}, \eta\right)=r^{-\mu} \tilde{\sigma}_{\partial}(\mathfrak{A})\left(r, x^{\prime}, y, r \rho, \xi^{\prime}, r \eta\right),
\end{gathered}
$$

where $\tilde{\sigma}_{\psi}(\mathfrak{A})(r, x, y, \tilde{\rho}, \xi, \tilde{\eta})$ and $\tilde{\sigma}_{\partial}(\mathfrak{A})\left(r, x^{\prime}, y, \tilde{\rho}, \xi^{\prime}, \tilde{\eta}\right)$, are of the same nature as the 'usual' homogeneous interior and boundary symbols, respectively, but are smooth up to $r=0$.

Theorem 1.4.5. $\mathfrak{A} \quad \in \quad \mathfrak{Y}^{\mu, d}(\mathbb{W} ; \boldsymbol{v})$ and $\mathfrak{B} \quad \in \quad \mathfrak{Y}^{\nu, e}(\mathbb{W} ; \boldsymbol{w}), \boldsymbol{v}=$ $\left(G_{-}, G_{+} ; J_{-}, J_{+}\right), \boldsymbol{w}=\left(\tilde{G}_{-}, G_{-} ; \tilde{J}_{-}, J_{-}\right)$entail $\mathfrak{A} \mathfrak{B} \in \mathfrak{Y}^{\mu+\nu, h}(\mathbb{W} ; \boldsymbol{v} \circ \boldsymbol{w})$ for $\boldsymbol{v} \circ \boldsymbol{w}=\left(\tilde{G}_{-}, G_{+} ; \tilde{J}_{-}, J_{+}\right)$, and $h$ as in Theorem 1.2.1, and we have

$$
\sigma(\mathfrak{A} \mathfrak{B})=\sigma(\mathfrak{A}) \sigma(\mathfrak{B})
$$

(with componentwise composition).

Remark 1.4.6. In the following considerations the relations between operators on $\mathbb{W}$ and $\mathbb{V}$ will play some role. Note that there is an analogue of Definition 1.4.1 for the case without boundary which gives us the operator spaces $\mathfrak{Y}^{\mu}(\mathbb{V} ; \boldsymbol{v})$ for bundle data $\boldsymbol{v}$ as before. Those operators are continuous as

$$
\mathfrak{B}: \begin{gathered}
\mathcal{W}^{s-\frac{1}{2}, \gamma-\frac{1}{2}}\left(\mathbb{V}, G_{-}\right) \\
\oplus \\
H^{s-1}\left(Y, J_{-}\right)
\end{gathered} \longrightarrow \begin{gathered}
\mathcal{W}^{s-\mu-\frac{1}{2}, \gamma-\mu-\frac{1}{2}}\left(\mathbb{V}, G_{+}\right) \\
\oplus \\
\oplus
\end{gathered}
$$


in this case for all s. The choice of the smoothness indices in (1.35) is motivated by (1.32) when we interpret $\mathfrak{Y}^{\mu}(\mathbb{V} ; \boldsymbol{v})$ as the space of lower right $2 \times 2$ corners of elements of $\mathfrak{Y}^{\mu, d}(\mathbb{W} ; \boldsymbol{v})$. The symbolic hierarchy only consists of two components, namely,

$$
\sigma(\mathfrak{B})=\left(\sigma_{\psi}(\mathfrak{B}), \sigma_{\wedge}(\mathfrak{B})\right) .
$$

Remark 1.4.7. The assumption that $W$ is compact was made for convenience. The essential elements of Definition 1.4.3 apply also in the non-compact case when we assume $X$ to be compact. An example is $W:=X^{\triangle} \times \mathbb{R}^{q}$ with $V=(\partial X)^{\triangle} \times \mathbb{R}^{q}$; the associated, stretched manifolds in this case are

$$
\mathbb{W}=\overline{\mathbb{R}}_{+} \times X \times \mathbb{R}^{q} \text { and } \mathbb{V}=\overline{\mathbb{R}}_{+} \times \partial X \times \mathbb{R}^{q},
$$

respectively. The corresponding edge operator spaces will be again denoted by $\mathfrak{Y}^{\mu, d}(\mathbb{W} ; \boldsymbol{v})$. Instead of (1.32) we have continuity between corresponding 'comp' and 'loc' versions of the involved spaces (where 'comp' and 'loc' in the example (1.36) refers to $(r, y)$; also in general the supports in the 'comp' case may intersect $\mathbb{W}_{\text {sing }}$ and $\mathbb{V}_{\text {sing }}$, respectively). Moreover, there is an analogue of Theorem 1.4.5 when one of the factors is properly supported in an obvious sense, or when we assume a localising function between the factors.

Remark 1.4.8. Another useful generalisation of $\mathfrak{Y}^{\mu, d}(\mathbb{W} ; \boldsymbol{v})$ is the space of all operators of the form

$$
\left\{\operatorname{diag}\left(1, R_{J_{+}}^{\rho}\right) \mathfrak{A} \operatorname{diag}\left(1, R_{J_{-}}^{-\sigma}\right): \mathfrak{A} \in \mathfrak{Y}^{\mu, d}(\mathbb{W} ; \boldsymbol{v})\right\}
$$

for any fixed reals $\rho, \sigma$, where $R_{J}^{\nu}$ is any elliptic element of $L_{\mathrm{cl}}^{\nu}(Y, J)$ that is assumed to be a reduction of orders by $\nu$ when $Y$ is compact (i.e., induces isomorphisms $R_{J}^{\nu}: H^{s}(Y, J) \longrightarrow H^{s-\nu}(Y, J)$ for all $\left.s \in \mathbb{R}\right)$. If we assume the bundles $J_{ \pm}$to be direct sums of bundles $J_{-}=\oplus_{i=1}^{k} J_{-, i}, J_{+}=\oplus_{j=1}^{l} J_{+, j}$ we can also modify (1.37) by replacing $R_{J_{-}}^{-\sigma}$ and $R_{J_{+}}^{\rho}$ by $\operatorname{diag}\left(R_{J_{-, i}}^{\sigma_{i}}: i=1, \ldots, k\right)$ and $\operatorname{diag}\left(R_{J_{+}, j}^{\rho_{j}}: j=1, \ldots, l\right)$. For simplicity, we denote again the operator spaces (1.37) by $\mathfrak{Y}^{\mu, d}(\mathbb{W} ; \boldsymbol{v})$, where $\mu$ is the dominating order in the upper left corner, while the other orders are known in the concrete context. The basic definitions and assertions, especially, on ellipticity, have straightforward extensions to that case. Clearly, there are other variants of choosing order shifts by composing with diagonal matrices, where the other entries are elliptic in the edge algebra on $\mathbb{W}$ (without extra conditions on $\mathbb{V}$ and $Y$ ) and on $\mathbb{V}$ (without extra conditions on $Y$ ).

\section{New quantisations on closed manifolds}

\subsection{Ellipticity and reduction to the edge}

Definition 2.1.1. An element $\mathfrak{A} \in \mathfrak{Y}^{\mu, d}(\mathbb{W})$ is called elliptic if the following conditions are satisfied: 
(i) $\sigma_{\psi}(\mathfrak{A}) \neq 0$ on $T^{*}\left(\mathbb{W}_{\text {reg }}\right) \backslash 0$ and, locally near $\mathbb{W}_{\text {sing }}, \tilde{\sigma}_{\psi}(\mathfrak{A})(r, x, y, \tilde{\rho}, \xi, \tilde{\eta}) \neq 0$ for $(\tilde{\rho}, \xi, \tilde{\eta}) \neq 0$, up to $r=0$;

(ii) $\sigma_{\partial}(\mathfrak{A})$ is a family of isomorphisms between the spaces occurring in the relation (1.21) for any $s>\max (\mu, d)-\frac{1}{2}$ (where $\left(x^{\prime}, \xi^{\prime}\right)$ is to be replaced by points on $\left.T^{*}\left(\partial \mathbb{W}_{\text {reg }}\right) \backslash 0\right)$, and, locally near $\partial \mathbb{W}_{\text {sing }}, \tilde{\sigma}_{\partial}(\mathfrak{A})\left(r, x^{\prime}, y, \tilde{\rho}, \xi^{\prime}, \tilde{\eta}\right)$ for $\left(\tilde{\rho}, \xi^{\prime}, \tilde{\eta}\right) \neq 0$ is a family of such isomorphisms, up to $r=0$;

(iii) The operators (1.33) form a family of isomorphisms for all $(y, \eta) \in T^{*} Y \backslash 0$, for any $s>\max (\mu, d)-\frac{1}{2}$.

Theorem 2.1.2. Let $\mathfrak{A} \in \mathfrak{Y}^{\mu, d}(\mathbb{W} ; \boldsymbol{v}), \boldsymbol{v}:=\left(G_{-}, G_{+} ; J_{-}, J_{+}\right)$be an elliptic operator. Then

(i) $\mathfrak{A}$ has a parametrix $\mathfrak{P} \in \mathfrak{Y}^{-\mu,(d-\mu)^{+}}\left(\mathbb{W} ; \boldsymbol{v}^{-1}\right), \boldsymbol{v}^{-1}:=\left(G_{+}, G_{-} ; J_{+}, J_{-}\right)$, i.e.,

$$
\mathfrak{P A}-\mathfrak{I} \in \mathfrak{Y}^{-\infty, d_{l}}\left(\mathbb{W} ; \boldsymbol{v}_{l}\right), \mathfrak{A} \mathfrak{P}-\mathfrak{I} \in \mathfrak{Y}^{-\infty, d_{r}}\left(\mathbb{W} ; \boldsymbol{v}_{r}\right)
$$

for $d_{l}$ and $d_{r}$ as in Theorem 1.2.2, $\boldsymbol{v}_{l}:=\left(G_{-}, G_{-} ; J_{-}, J_{-}\right), \boldsymbol{v}_{r}:=$ $\left(G_{+}, G_{+} ; J_{+}, J_{+}\right)$;

(ii) $\mathfrak{A}$ defines a Fredholm operator (1.32) for every $s>\max (\mu, d)-\frac{1}{2}$.

Consider elements $\mathfrak{A}_{j} \in \mathfrak{Y}^{\mu, d}\left(\mathbb{W} ; \boldsymbol{v}_{j}\right), \boldsymbol{v}_{j}:=\left(G_{-}^{j}, G_{+}^{j} ; J_{-}^{j}, J_{+}^{j}\right), j=1,2$, written in $3 \times 3$-block matrix form, with elements $\mathcal{A}_{j}$ belonging to $\mathcal{B}^{\mu, d}\left(\mathbb{W}_{\text {reg }} ; G_{-}^{j}, G_{+}^{j}\right)$ in the $2 \times 2$-upper left corners. Assuming $\mathcal{A}:=\mathcal{A}_{1}=\mathcal{A}_{2}$ (with $G_{ \pm}^{j}=: G_{ \pm}$) we can also write

$$
\mathfrak{A}_{j}=\left(\begin{array}{cc}
\mathcal{A} & \mathcal{K}_{j} \\
\mathcal{T}_{j} & \mathcal{Q}_{j}
\end{array}\right): \begin{gathered}
\mathcal{W}^{s, \gamma}\left(\mathbb{W} ; G_{-}\right) \\
\oplus \\
H^{s-1}\left(Y, J_{-}^{j}\right)
\end{gathered} \longrightarrow \begin{gathered}
\mathcal{W}^{s-\mu, \gamma-\mu}\left(\mathbb{W} ; G_{+}\right) \\
\oplus \\
H^{s-\mu-1}\left(Y, J_{+}^{j}\right)
\end{gathered}
$$

$j=1,2$.

Let $\mathfrak{A}_{j}$ be elliptic in the sense of Definition 2.1.1 and compare the Fredholm indices ind $\mathfrak{A}_{j}, j=1,2$, by an analogue of the Agranovich-Dynin formula in boundary value problems.

First observe that the operators

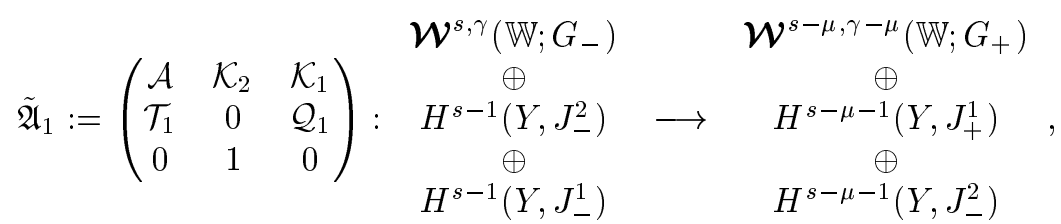




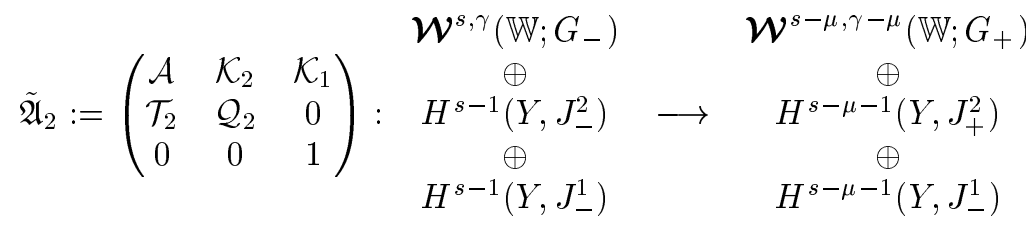

are Fredholm operators for $s>\max (\mu, d)-\frac{1}{2}$.

If

$$
\mathfrak{P}_{1}=\left(\begin{array}{ll}
\mathcal{P}_{1} & \mathcal{L}_{1} \\
\mathcal{S}_{1} & \mathcal{B}_{1}
\end{array}\right)
$$

denotes a parametrix of $\mathfrak{A}_{1}$ which we have by Theorem 2.1 .2 we find (by a purely algebraic calculation) a parametrix $\tilde{\mathfrak{P}}_{1}$ of $\tilde{\mathfrak{A}}_{1}$ in the form

$$
\tilde{\mathfrak{P}}_{1}=\left(\begin{array}{ccc}
\mathcal{P}_{1} & \mathcal{L}_{1} & -\mathcal{P}_{1} \mathcal{K}_{2} \\
0 & 0 & 1 \\
\mathcal{S}_{1} & \mathcal{B}_{1} & -\mathcal{S}_{1} \mathcal{K}_{2}
\end{array}\right)
$$

By virtue of Theorem 2.1.2 (i) and Theorem 1.4.5 we then obtain mod $\mathfrak{Y}^{-\infty, h}(\mathbb{W} ; \tilde{\boldsymbol{v}})$

$$
\tilde{\mathfrak{A}}_{2} \tilde{\mathfrak{P}}_{1}=\left(\begin{array}{ccc}
1 & 0 & 0 \\
\mathcal{T}_{2} \mathcal{P}_{1} & \mathcal{T}_{2} \mathcal{L}_{1} & -\mathcal{T}_{2} \mathcal{P}_{1} \mathcal{K}_{2}+\mathcal{Q}_{2} \\
\mathcal{S}_{1} & \mathcal{B}_{1} & -\mathcal{S}_{1} \mathcal{K}_{2}
\end{array}\right) \in \mathfrak{Y}^{0, h}(\mathbb{W} ; \tilde{\boldsymbol{v}}) ;
$$

here, $\tilde{\boldsymbol{v}}=\left(G_{+}, G_{+} ; J_{+}^{1} \oplus J_{-}^{2}, J_{+}^{2} \oplus J_{-}^{1}\right)$, and $h$ is as in Theorem 1.2.1. In particular, the operator

$$
R:=\left(\begin{array}{cc}
\mathcal{T}_{2} \mathcal{L}_{1} & -\mathcal{T}_{2} \mathcal{P}_{1} \mathcal{K}_{2}+\mathcal{Q}_{2} \\
\mathcal{B}_{1} & -\mathcal{S}_{1} \mathcal{K}_{2}
\end{array}\right)
$$

belongs to $L_{\mathrm{cl}}^{0}\left(Y, J_{+}^{1} \oplus J_{-}^{2}, J_{+}^{2} \oplus J_{-}^{1}\right)$ and is elliptic. Thus

$$
R: \begin{gathered}
H^{s-1}\left(Y, J_{+}^{1}\right) \\
\oplus \\
H^{s-1}\left(Y, J_{-}^{2}\right)
\end{gathered} \longrightarrow \begin{gathered}
H^{s-1}\left(Y, J_{+}^{2}\right) \\
\oplus \\
H^{s-1}\left(Y, J_{-}^{1}\right)
\end{gathered}
$$

is a Fredholm operator with ind $R$ being independent of $s$.

Theorem 2.1.3 (first version of the Agranovich-Dynin formula). Let $\mathfrak{A}_{1}$ and $\mathfrak{A}_{2}$ be elliptic edge operators of the class $\mathfrak{Y}^{\mu, d}(\mathbb{W})$ with the same $2 \times 2$-upper left corner $\mathcal{A}$. Then we have

$$
\text { ind } \mathfrak{A}_{2}-\text { ind } \mathfrak{A}_{1}=\text { ind } R
$$

for the elliptic operator (2.3) on the edge $Y$.

Proof. We have ind $\mathfrak{A}_{j}=$ ind $\tilde{\mathfrak{A}}_{j}$ for $j=1,2$, and ind $\tilde{\mathfrak{P}}_{1}=-$ ind $\tilde{\mathfrak{A}}_{1}$. The formula (2.2) gives us ind $\tilde{\mathfrak{A}}_{2} \tilde{\mathfrak{P}}_{1}=$ ind $R$ which yields ind $\mathfrak{A}_{2}-$ ind $\mathfrak{A}_{1}=$ ind $R$. 
We now pass to another application of this algebraic construction. Let $\mathfrak{A}_{j} \in$ $\mathfrak{Y}^{\mu, d}\left(\mathbb{W} ; \boldsymbol{v}_{j}\right), \boldsymbol{v}_{j}:=\left(G_{-}^{j}, G_{+}^{j} ; J_{-}^{j}, J_{+}^{j}\right), j=1,2$ be elliptic elements which are of $3 \times 3$-block matrix form. In contrast to the construction before we now only assume that the upper left corners $A:=\left(\mathfrak{A}_{1}\right)_{11}=\left(\mathfrak{A}_{2}\right)_{11}$ are the same for $j=1,2$, and write

$$
\mathfrak{A}_{j}=\left(\begin{array}{cc}
A & \mathfrak{K}_{j} \\
\mathfrak{T}_{j} & \mathfrak{Q}_{j}
\end{array}\right): \begin{gathered}
\mathcal{W}^{s, \gamma}(\mathbb{W}) \\
\mathcal{V}^{s-\frac{1}{2}, \gamma-\frac{1}{2}}\left(\mathbb{V} ; G_{-}^{j}, J_{-}^{j}\right)
\end{gathered} \quad \begin{gathered}
\mathcal{W}^{s-\mu, \gamma-\mu}(\mathbb{W}) \\
\mathcal{V}^{s-\mu-\frac{1}{2}, \gamma-\mu-\frac{1}{2}}\left(\mathbb{V} ; G_{+}^{j}, J_{+}^{j}\right)
\end{gathered}
$$

where $\mathcal{V}^{s-\frac{1}{2}, \gamma-\frac{1}{2}}(\mathbb{V} ; G, J):=\begin{gathered}\mathcal{W}^{s-\frac{1}{2}, \gamma-\frac{1}{2}}(\mathbb{V}, G) \\ H^{s-1}(Y, J)\end{gathered}$ for bundles $G$ on $V$ and $J$ on $Y$; further, when we write $\left(\left(\mathfrak{A}_{j}\right)_{k l}\right)_{k, l=1,2,3}$, we have $\mathfrak{K}_{j}=\left(\left(\mathfrak{A}_{j}\right)_{12},\left(\mathfrak{A}_{j}\right)_{13}\right), \mathfrak{T}_{j}=$ ${ }^{\mathrm{t}}\left(\left(\mathfrak{A}_{j}\right)_{21},\left(\mathfrak{A}_{j}\right)_{23}\right), \mathfrak{Q}_{j}=\left(\left(\mathfrak{A}_{j}\right)_{k l}\right)_{k, l=2,3}$. Similarly as before we now form the extended operators

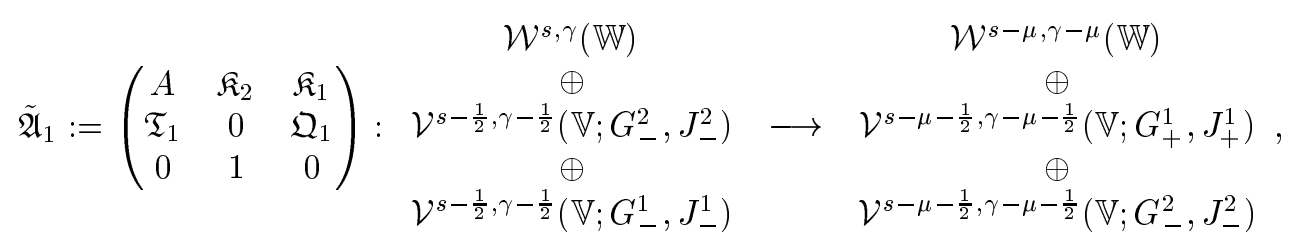

and

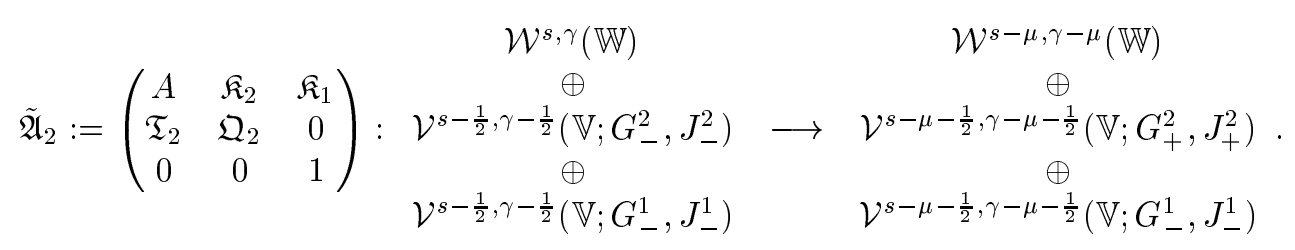

Now if we form a parametrix $\mathfrak{P}_{1}=\left(\begin{array}{cc}P_{1} & \mathfrak{L}_{1} \\ \mathfrak{S}_{1} & \mathfrak{B}_{1}\end{array}\right)$ of $\mathfrak{A}_{1}$ we obtain a parametrix $\tilde{\mathfrak{P}}_{1}$ of $\tilde{\mathfrak{A}}_{1}$ of analogous form as (2.1) which gives us an elliptic operator

$$
\mathfrak{R}=\left(\begin{array}{cc}
\mathfrak{T}_{2} \mathfrak{L}_{1} & -\mathfrak{T}_{2} P_{1} \mathfrak{K}_{2}+\mathfrak{Q}_{2} \\
\mathfrak{B}_{1} & -\mathfrak{S}_{1} \mathfrak{K}_{2}
\end{array}\right)
$$

in $\mathfrak{Y}^{0}(\mathbb{V} ; \mathfrak{r})$ with bundle data $\mathfrak{r}=\left(G_{+}^{1} \oplus G_{-}^{2}, G_{+}^{2} \oplus G_{-}^{1} ; J_{+}^{1} \oplus J_{-}^{2}, J_{+}^{2} \oplus J_{-}^{1}\right)$. Moreover, we have

$$
\mathfrak{R}: \begin{gathered}
\mathcal{V}^{s-\frac{1}{2}, \gamma-\frac{1}{2}}\left(\mathbb{V} ; G_{+}^{1}, J_{+}^{1}\right) \\
\mathcal{V}^{s-\frac{1}{2}, \gamma-\frac{1}{2}}\left(\mathbb{V} ; G_{-}^{2}, J_{-}^{2}\right)
\end{gathered} \longrightarrow \begin{gathered}
\mathcal{V}^{s-\frac{1}{2}, \gamma-\frac{1}{2}}\left(\mathbb{V} ; G_{+}^{2}, J_{+}^{2}\right) \\
\mathcal{V}^{s-\frac{1}{2}, \gamma-\frac{1}{2}}\left(\mathbb{V} ; G_{-}^{1}, J_{-}^{1}\right)
\end{gathered}
$$


Theorem 2.1.4 (second version of the Agranovich-Dynin formula). Let $\mathfrak{A}_{1}$ and $\mathfrak{A}_{2}$ be elliptic edge operators of the class $\mathfrak{Y}^{\mu, d}(\mathbb{W})$ with the same $1 \times 1$-upper left corner A. Then we have

$$
\text { ind } \mathfrak{A}_{2}-\text { ind } \mathfrak{A}_{1}=\text { ind } \mathfrak{R}
$$

for the elliptic operator (2.4) on $\mathbb{V}$.

Proof. The arguments are analogous to the proof of Theorem 2.1.3. We form $\tilde{\mathfrak{A}}_{2} \tilde{\mathfrak{P}}_{1}$ which is again a triangular matrix, similarly as $(2.2)$, now with $\mathfrak{R}$ in the lower right corner. This gives us immediately the asserted index expression.

\subsection{Boundary value problems with fictitious edges}

We now apply the constructions of the preceding sections to the case of a compact $C^{\infty}$ manifold $X$ with boundary $\partial X$, where on the boundary we fix a closed compact $C^{\infty}$ manifold $Y$, regarded as a 'fictitious' edge. As is shown in [19] 'standard' elliptic boundary value problems for differential operators with differential boundary conditions can be quantised in a way that the new operators act in weighted edge Sobolev spaces and are Fredholm, either automatically for sufficiently large weights, or, after adding extra edge conditions for arbitrary weights. The number (more precisely, the difference of the numbers) of edge conditions of trace and potential type follows by applying relative index results for conormal symbols; all this up to a discrete set of exceptional weights. Similar results have been obtained before in [4] for the case of elliptic differential operators on a closed compact $C^{\infty}$ manifold $M$ with fictitious edge $Y \subset M$. Although for arbitrary elliptic pseudo-differential operators on $M$ (for the closed case) or pseudo-differential elliptic boundary value problems on $X$ (for the case with boundary) edge-quantisations with respect to $Y$ always exist, the explicit information on admissible weights or the number (and the computation) of extra edge conditions is extremely hard to deduce. Therefore, it is an interesting task to enlarge the classes of known examples. What concerns [4] the answers are given for elliptic differential operators on a closed $C^{\infty}$ manifold $M$ as well as for their pseudo-differential parametrices, using the fact that the edge calculus is closed under parametrix construction, and the parametrix for an elliptic edge problem is compatible with the parametrix of the elliptic operator on $M$ as a smooth manifold. What we do not reach in this construction are elliptic operators on $M$ which have not the transmission property with respect to any hypersurface $Y$ on $M$ (when $\operatorname{codim} Y=1$ ), as is typical for the case $M=\partial X$ for a manifold $X$ with boundary and the Dirichlet-Neumann operator on $M$ (which is of order 1 and with principal symbol equal to the absolute value of the covariable on $\partial X$, up to a constant). Also other elliptic operators on $\partial X$, obtained by reducing elliptic boundary value problems to the boundary are of that type (except for trivial cases). The program of the present section is to 
derive edge quantisations for such operators in general. For simplicity, we assume $Y$ to have a trivial normal bundle in $\partial X$; the general case can be treated as well, cf., analogously, the arguments in [4]. In the following section we give examples and specify the constructions, in particular, for the Dirichlet-Neumann operator. Let $A$ be an elliptic differential operator of order $2 m$ on $X$, and let

$$
\mathcal{A}:=\left(\begin{array}{c}
A \\
T
\end{array}\right): H^{s}(X) \longrightarrow \boldsymbol{H}^{s-2 m}(X)
$$

be the operator, representing an elliptic boundary value problem for $A$, i.e.,

$$
A u=f \text { in } X, T u=g \text { on } \partial X,
$$

where $T={ }^{\mathrm{t}}\left(T_{1}, \ldots, T_{m}\right)$ is a vector of trace operators of the form $T_{j}=\mathrm{r}_{\partial X} B_{j}$ for differential operators $B_{j}$ of order $\mu_{j} \leq 2 m-1$ given in a neighbourhood of $\partial X$, with smooth coefficients, where $T$ satisfies the Shapiro-Lopatinskij condition with respect to $A$. For abbreviation, in (2.6) we set

$$
\boldsymbol{H}^{s-2 m}(X):=H^{s-2 m}(X) \oplus \bigoplus_{j=1}^{m} H^{s-\mu_{j}-\frac{1}{2}}(\partial X) .
$$

Fix a $C^{\infty}$ submanifold $Y$ of $\partial X$ of codimension $b$, for simplicity, with a trivial normal bundle in $\partial X$. Then from [19] we have the following result:

Theorem 2.2.1. The operator (2.6) can be written as an elliptic edge boundary value problem (in the sense of Definition 2.1.1)

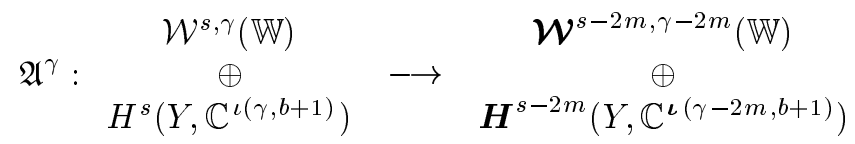

for every $\gamma \in \mathbb{R}$ with $\gamma-2 m>\frac{b+1}{2}, \gamma-2 m-\frac{b+1}{2} \notin \mathbb{N}, \gamma-\mu_{j}>\frac{b}{2}, \gamma-\mu_{j}-\frac{b}{2} \notin \mathbb{N}$, $s>\mu_{j}+\frac{1}{2}$ for $j=1, \ldots, m$, and

$$
\begin{gathered}
\iota(\gamma, b):=\#\left\{\alpha: \alpha \in \mathbb{N}^{b},|\alpha|<\gamma-\frac{b}{2}\right\}, \\
\boldsymbol{H}^{s-2 m}\left(Y, \mathbb{C}^{\iota(\gamma-2 m, b+1)}\right):=H^{s-2 m}\left(Y, \mathbb{C}^{\iota(\gamma-2 m, b+1)}\right) \\
\bigoplus_{j=1}^{m} H^{s-\mu_{j}-\frac{1}{2}}\left(Y, \mathbb{C}^{\iota\left(\gamma-\mu_{j}-\frac{1}{2}, b\right)}\right)
\end{gathered} ;
$$

concerning the other space on the right of (2.7), cf. the formula (1.13).

In other words, we have $\mathfrak{A}^{\gamma} \in \mathfrak{Y}^{2 m, d}(\mathbb{W} ; \boldsymbol{v})$ for $d=\max \left(\mu_{j}+1\right)_{j=1, \ldots, m}$, and $\boldsymbol{v}$ denotes a tuple of trivial bundles where the fibre dimensions of those on the boundary are $\iota(\gamma, b+1)$ and $\iota(\gamma-2 m, b+1)$, respectively (concerning notation, 
cf. also Remark 1.4.8).

Recall that the relation between (2.6) and (2.7) comes from isomorphisms of the kind

$$
\left(\begin{array}{ll}
E & L
\end{array}\right): \underset{H^{s}\left(Y, \mathbb{C}^{\iota(s, b+1)}\right)}{\stackrel{\mathcal{W}^{s, s}(\mathbb{W})}{\oplus}} \longrightarrow H^{s}(X)
$$

for $s \geq 0, s-\frac{b+1}{2} \notin \mathbb{N}$ where $X$ with the prescribed $Y$ on the boundary is identified with a manifold $W$ with edge $Y$ and boundary, and $\mathbb{W}$ is the associated stretched manifold. Similarly, we have isomorphisms

$$
\left(\begin{array}{ll}
E^{\prime} & L^{\prime}
\end{array}\right): \begin{aligned}
& \mathcal{W}^{s-\frac{1}{2}, s-\frac{1}{2}}(\mathbb{V}) \\
& H^{s-\frac{1}{2}}\left(Y, \mathbb{C}^{\iota\left(s-\frac{1}{2}, b\right)}\right)
\end{aligned} \longrightarrow H^{s-\frac{1}{2}}(\partial X)
$$

for all $s \geq 0, s-\frac{b}{2} \notin \mathbb{N}$. in this case $\partial X$ with the prescribed submanifold $Y$ on the boundary is identified with a manifold $V$ with edge $Y$, and $\mathbb{V}$ is the associated stretched manifold. Now $\mathfrak{A}^{\gamma}$ is just the result of a composition of $\mathcal{A}$ with isomorphisms of the kind (2.8), (2.9) or their inverses which are corresponding column matrices.

Let us now observe that the process of reducing edge-boundary value problems to the boundary has a simpler analogue in the classical context, the reduction to the boundary in the smooth case. In fact, let

$$
\mathcal{A}_{i}:=\left(\begin{array}{c}
A \\
T_{i}
\end{array}\right), i=1,2
$$

be two elliptic boundary value problems for the same elliptic differential operator $A$. Then from the pseudo-differential calculus of boundary value problems with the transmission property at the boundary we can calculate a parametrix $\mathcal{P}_{1}:=$ $\left(\begin{array}{ll}P_{1} & K_{1}\end{array}\right)$ within the calculus. This allows us to form

$$
\mathcal{A}_{2} \mathcal{P}_{1}=\left(\begin{array}{cc}
1 & 0 \\
T_{2} P_{1} & T_{2} K_{1}
\end{array}\right)
$$

with equality modulo compact (in fact, smoothing) remainders. The operator $T_{2} K_{1}=: R$ is then pseudo-differential and elliptic on the boundary $\partial X$. We now observe the same process along the lines of transforming $\mathcal{A}_{i}$ to corresponding elliptic edge boundary value problems $\mathfrak{A}_{i}^{\gamma}$, according to Theorem 2.2.1. Then

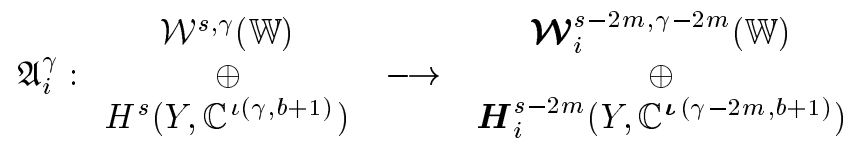

for

$$
\mathcal{W}_{i}^{s-2 m, \gamma-2 m}(\mathbb{W}):=\mathcal{W}^{s-2 m, \gamma-2 m}(\mathbb{W}) \oplus \bigoplus_{j=1}^{m} \mathcal{W}^{s-\mu_{i, j}-\frac{1}{2}, \gamma-\mu_{i, j}-\frac{1}{2}}(\mathbb{V})
$$




$$
\boldsymbol{H}_{i}^{s-2 m}\left(Y, \mathbb{C}^{\iota(\gamma-2 m, b+1)}\right):=\begin{gathered}
H^{s-2 m}\left(Y, \mathbb{C}^{\iota(\gamma-2 m, b+1)}\right) \\
\bigoplus_{j=1}^{m} H^{s-\mu_{i, j}-\frac{1}{2}}\left(Y, \mathbb{C}^{\iota\left(\gamma-\mu_{i, j}-\frac{1}{2}, b\right)}\right)
\end{gathered} .
$$

The notation $\mu_{i, j}$ in connection with the components of $T_{i}={ }^{\mathrm{t}}\left(T_{i, 1}, \ldots, T_{i, m}\right)$ has an analogous meaning as in Remark 1.1.9, namely as the orders of the differential operators involved in $T_{i, j}$.

Remark 2.2.2. The assumptions on the weight $\gamma$ in Theorem 2.2 .1 depend on $i$; however, they exclude only discrete sets $\Sigma_{i}$ of exceptional weights. In the following construction we assume $\gamma \notin \Sigma_{1} \cup \Sigma_{2}$.

The upper left corner of $\mathfrak{A}_{i}^{\gamma}$ again coincides with the operator $A$, now realised on a corresponding weighted edge Sobolev space. Applying the construction for Theorem 2.1.4 we can form the operator

$$
\mathfrak{A}_{2}^{\gamma} \mathfrak{P}_{1}^{\gamma}=\left(\begin{array}{cc}
1 & 0 \\
\mathfrak{T} & \mathfrak{R}^{\gamma}
\end{array}\right)
$$

where $\mathfrak{P}_{1}^{\gamma}$ denotes a parametrix of $\mathfrak{A}_{1}^{\gamma}$ in the edge algebra of boundary value problems, and $\mathfrak{R}^{\gamma}$ is an elliptic operator in the edge algebra on the boundary $\mathbb{V}$.

Theorem 2.2.3. The operator $\mathfrak{R}^{\gamma}$ is an edge-representation of the elliptic operator $R$ on the boundary in a similar spirit as in the edge-representations of [4], where we have ind $R=$ ind $\mathfrak{R}^{\gamma}$.

\subsection{Edge quantisation of the Dirichlet-Neumann operator}

We now apply the scheme of the preceding section to establish the edge quantisation of a specific elliptic operator on the boundary $\partial X$ of a compact $C^{\infty}$ manifold $X$ with boundary, namely, the operator which appears when we reduce the Neumann problem for the Laplacian to the boundary by means of the Dirichlet problem (which is the motivation of the notation). In other words, in (2.10) we consider $A=\Delta$ and take $T_{1}=D$ the Dirichlet, and $T_{2}=N$ the Neumann problem. What we want to do in addition to the information of the previous section is to see in more explicit terms the structure of the operators $\mathfrak{R}^{\gamma}$ associated with $R=T_{2} K_{1}$. By 'explicit' we mean the symbolic structures of the corresponding localised operators. Let us first localise the Dirichlet problem $\mathcal{A}_{1}$ in the half-space $\overline{\mathbb{R}}_{+}^{n}=\left\{x=\left(x_{1}, \ldots, x_{n}\right) \in \mathbb{R}^{n}: x_{n} \geq 0\right\}$. In the following calculations it will be convenient to write $t:=x_{n}$ and $\tau:=\xi_{n}$. We have $\sigma_{\psi}\left(\mathcal{A}_{1}\right)(x, \xi)=\sigma_{\psi}(A)(x, \xi)=-|\xi|^{2}$ and

$$
\sigma_{\partial}\left(\mathcal{A}_{1}\right)\left(\xi^{\prime}\right)=\left(\begin{array}{c}
-\left|\xi^{\prime}\right|^{2}-D_{t}^{2} \\
\mathrm{r}_{\{t=0\}}
\end{array}\right): H^{s}\left(\mathbb{R}_{+}\right) \longrightarrow \stackrel{H^{s-2}\left(\mathbb{R}_{+}\right)}{\longrightarrow} \underset{\mathbb{C}}{\oplus}
$$


for $s>\frac{3}{2},\left(x^{\prime}, \xi^{\prime}\right) \in T^{*} \mathbb{R}^{n-1} \backslash 0$. We want to calculate $\sigma_{\partial}\left(\mathcal{A}_{1}\right)^{-1}\left(\xi^{\prime}\right)$. To this end we write $-\left|\xi^{\prime}\right|^{2}-\tau^{2}=-l_{-}\left(\xi^{\prime}, \tau\right) l_{+}\left(\xi^{\prime}, \tau\right)$ for $l_{ \pm}\left(\xi^{\prime}, \tau\right):=\left|\xi^{\prime}\right| \pm i \tau$ for any fixed $\xi^{\prime} \neq 0$. We then have

$$
-\left|\xi^{\prime}\right|^{2}-D_{t}^{2}=-\mathrm{op}^{+}\left(l_{-}\right)\left(\xi^{\prime}\right) \mathrm{op}^{+}\left(l_{+}\right)\left(\xi^{\prime}\right)
$$

This allows us to write

$$
\sigma_{\partial}\left(\mathcal{A}_{1}\right)\left(\xi^{\prime}\right)=-\left(\begin{array}{cc}
\mathrm{op}^{+}\left(l_{-}\right)\left(\xi^{\prime}\right) & 0 \\
0 & 1
\end{array}\right)\left(\begin{array}{c}
\mathrm{op}^{+}\left(l_{+}\right)\left(\xi^{\prime}\right) \\
\mathrm{r}_{\{t=0\}}
\end{array}\right) .
$$

We have, since $l_{-}$is a minus-symbol (in the terminology of Eskin [6]), $\left(\mathrm{op}^{+}\left(l_{-}\right)\left(\xi^{\prime}\right)\right)^{-1}=\mathrm{op}^{+}\left(l_{-}^{-1}\right)\left(\xi^{\prime}\right)$. Moreover, if we form the map

$$
k\left(\xi^{\prime}\right): \mathbb{C} \longrightarrow \mathcal{S}\left(\overline{\mathbb{R}}_{+}\right)
$$

by $k\left(\xi^{\prime}\right) c:=c e^{-t\left|\xi^{\prime}\right|}$ we have

$$
\left(\begin{array}{c}
\mathrm{op}^{+}\left(l_{+}\right)\left(\xi^{\prime}\right) \\
\mathrm{r}_{\{t=0\}}
\end{array}\right)^{-1}=\left(\mathrm{op}^{+}\left(l_{+}^{-1}\right)\left(\xi^{\prime}\right) k\left(\xi^{\prime}\right)\right)
$$

which follows by a straightforward calculation. This gives us

$$
\begin{aligned}
& \sigma_{\partial}\left(\mathcal{A}_{1}\right)^{-1}\left(\xi^{\prime}\right)=-\left(\mathrm{op}^{+}\left(l_{+}^{-1}\right)\left(\xi^{\prime}\right) k\left(\xi^{\prime}\right)\right)\left(\begin{array}{cc}
\mathrm{op}^{+}\left(l_{-}^{-1}\right)\left(\xi^{\prime}\right) & 0 \\
0 & 1
\end{array}\right) \\
& =-\left(\mathrm{op}^{+}\left(l_{+}^{-1}\right)\left(\xi^{\prime}\right) \mathrm{op}^{+}\left(l_{-}^{-1}\right)\left(\xi^{\prime}\right) k\left(\xi^{\prime}\right)\right) .
\end{aligned}
$$

Note that op ${ }^{+}\left(l_{+}^{-1}\right)\left(\xi^{\prime}\right) \mathrm{op}^{+}\left(l_{-}^{-1}\right)\left(\xi^{\prime}\right)=\mathrm{op}^{+}\left(l_{+}^{-1} l_{-}^{-1}\right)\left(\xi^{\prime}\right)+g\left(\xi^{\prime}\right)$ for some Green operator $g\left(\xi^{\prime}\right)$ on $\mathbb{R}_{+}$(in the sense of the boundary symbolic calculus).

The boundary symbol of $\mathcal{A}_{2}$ is equal to

$$
\sigma_{\partial}\left(\mathcal{A}_{2}\right)\left(\xi^{\prime}\right)=\left(\begin{array}{c}
-\left|\xi^{\prime}\right|^{2}-D_{t}^{2} \\
\mathrm{r}_{\{t=0\}} \frac{d}{d t}
\end{array}\right): H^{s}\left(\mathbb{R}_{+}\right) \longrightarrow \begin{gathered}
H^{s-2}\left(\mathbb{R}_{+}\right) \\
\underset{\mathbb{C}}{\oplus}
\end{gathered}
$$

We then have an explicit expression for $\sigma_{\partial}\left(\mathcal{A}_{2} \mathcal{P}_{1}\right)$ when $\mathcal{P}_{1}$ denotes a parametrix of $\mathcal{A}_{1}$, namely

$$
\sigma_{\partial}\left(\mathcal{A}_{2} \mathcal{P}_{1}\right)=\sigma_{\partial}\left(\mathcal{A}_{2}\right) \sigma_{\partial}\left(\mathcal{A}_{1}\right)^{-1}
$$

In order to calculate the principal symbol of the Dirichlet-Neumann operator $R$ it suffices to look at the lower right corner of (2.15), and it follows that

$$
\sigma_{\psi}(R)\left(\xi^{\prime}\right)=\mathrm{r}_{\{t=0\}} \frac{d}{d t} k\left(\xi^{\prime}\right)=-\left|\xi^{\prime}\right| .
$$

Let us now recall a relation between 'standard' elliptic boundary value problems and elliptic edge problems in the sense of Definition 2.1.1 for the case of the 
operators (2.10) which represent the Dirichlet and the Neumann problem in the half-space $\overline{\mathbb{R}}_{+}^{n}$. On $\partial \overline{\mathbb{R}}_{+}^{n}=\mathbb{R}^{n-1}$ we prescribe a 'fictitious' edge $Y$ that we assume to be the coordinate hyperplane defined by $\tilde{x}^{\prime}:=\left\{x_{q+1}, \ldots, x_{n-1}\right\}$ for some $1 \leq q<$ $n-1$; then $x^{\prime}=\left(y, \tilde{x}^{\prime}\right)$ for $y=\left(x_{1}, \ldots, x_{q}\right)$, and $x=(y, \tilde{x})$ for $\tilde{x}:=\left(x_{q+1}, \ldots, x_{n}\right)$. The operator $\mathcal{A}_{1}=\left(\begin{array}{c}\Delta \\ D\end{array}\right)$ is first continuous in the sense

$$
\mathcal{A}_{1}: H^{s}\left(\mathbb{R}_{+}^{n}\right) \longrightarrow \underset{H^{s-\frac{1}{2}}\left(\mathbb{R}^{n-1}\right)}{H_{+}^{s-2}\left(\mathbb{R}_{+}^{n}\right)} ;
$$

in the following we always assume $s>\frac{3}{2}$.

Introducing polar coordinates $\tilde{x} \rightarrow(r, \phi), \overline{\mathbb{R}}_{+}^{b+1} \backslash\{0\} \rightarrow \mathbb{R}_{+} \times S_{+}^{b}$ for $b=n-1-$ $q, S_{+}^{b}=S^{b} \cap\left\{x_{n} \geq 0\right\}$, and $\tilde{x}^{\prime} \rightarrow\left(r, \phi^{\prime}\right), \mathbb{R}^{b} \backslash\{0\} \rightarrow \mathbb{R}_{+} \times S^{b-1}$, we can identify our Sobolev spaces with weighted edge Sobolev spaces on the (stretched) manifolds with edge $\mathbb{R}^{q}$

$$
\mathbb{W}:=\overline{\mathbb{R}}_{+} \times S_{+}^{b} \times \mathbb{R}^{q} \text { and } \mathbb{V}:=\overline{\mathbb{R}}_{+} \times S^{b-1} \times \mathbb{R}^{q},
$$

respectively, cf. the formula (1.36). Observe that, according to the general notation,

$$
\begin{gathered}
\mathbb{W}_{\text {reg }}=\mathbb{R}_{+} \times S_{+}^{b} \times \mathbb{R}^{q}, \mathbb{W}_{\text {sing }}=\{0\} \times S_{+}^{b} \times \mathbb{R}^{q} \text { and } \\
\mathbb{V}_{\text {reg }}=\mathbb{R}_{+} \times S^{b-1} \times \mathbb{R}^{q}, \mathbb{V}_{\text {sing }}=\{0\} \times S^{b-1} \times \mathbb{R}^{q} .
\end{gathered}
$$

We then have the weighted edge Sobolev spaces $\mathcal{W}^{s, \gamma}(\mathbb{W})=\mathcal{W}^{s}\left(\mathbb{R}^{q}, \mathcal{K}^{s, \gamma}\left(\left(S_{+}^{b}\right)^{\wedge}\right)\right)$ and $\mathcal{W}^{s, \gamma}(\mathbb{V})=\mathcal{W}^{s}\left(\mathbb{R}^{q}, \mathcal{K}^{s, \gamma}\left(\left(S^{b-1}\right)^{\wedge}\right)\right)$, respectively. The identifications are given by the following theorem, proved in [4] (cf. also [19] for the case with boundary):

Theorem 2.3.1. For every $s>\frac{b+1}{2}, s-\frac{b+1}{2} \notin \mathbb{N}$, there are natural isomorphisms

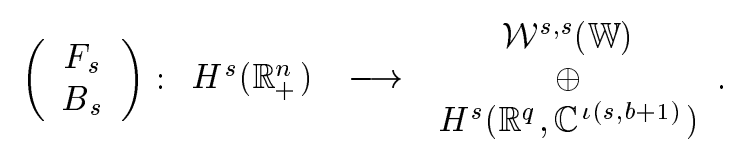

Similarly, for every $s>\frac{b}{2}, s-\frac{b}{2} \notin \mathbb{N}$, there are natural isomorphisms

$$
\left(\begin{array}{c}
F_{s}^{\prime} \\
B_{s}^{\prime}
\end{array}\right): H^{s}\left(\mathbb{R}^{n-1}\right) \longrightarrow \begin{gathered}
\mathcal{W}^{s, s}(\mathbb{V}) \\
H^{s}\left(\mathbb{R}^{q}, \mathbb{C}^{\iota(s, b)}\right)
\end{gathered} .
$$

By 'natural' we mean the constructions of [4]; they are not canonical but depend on cut-off constructions, etc. Let

$$
\left(\begin{array}{ll}
E_{s} & L_{s}
\end{array}\right):=\left(\begin{array}{c}
F_{s} \\
B_{s}
\end{array}\right)^{-1} \text { and }\left(\begin{array}{ll}
E_{s}^{\prime} & L_{s}^{\prime}
\end{array}\right):=\left(\begin{array}{c}
F_{s}^{\prime} \\
B_{s}^{\prime}
\end{array}\right)^{-1}
$$


denote the respective inverses. From (2.16) we then pass to

$$
\mathfrak{A}_{1}^{s}:=\left(\begin{array}{cc}
F_{s-2} & 0 \\
0 & F_{s-\frac{1}{2}}^{\prime} \\
B_{s-2} & 0 \\
0 & B_{s-\frac{1}{2}}^{\prime}
\end{array}\right)\left(\begin{array}{c}
\Delta \\
D
\end{array}\right)\left(\begin{array}{ll}
E_{s} & L_{s}
\end{array}\right)=\left(\begin{array}{cc}
F_{s-2} \Delta E_{s} & F_{s-2} \Delta L_{s} \\
F_{s-\frac{1}{2}}^{\prime} D E_{s} & F_{s-\frac{1}{2}}^{\prime} D L_{s} \\
0 & B_{s-2} \Delta L_{s} \\
0 & B_{s-\frac{1}{2}}^{\prime} D L_{s}
\end{array}\right)
$$

which represents continuous operators

$$
\begin{gathered}
\mathfrak{A}_{1}^{s}: \begin{array}{c}
\mathcal{W}^{s, s}(\mathbb{W}) \\
H^{s}\left(\mathbb{R}^{q}, \mathbb{C}^{\iota(s, b+1)}\right)
\end{array} \longrightarrow \\
\boldsymbol{H}_{1}^{s-2}\left(\mathbb{R}^{q}, \mathbb{C}^{\iota(s-2, b+1)}\right)
\end{gathered}
$$

for

$$
\mathcal{W}_{1}^{s-2, s-2}(\mathbb{W}):=\begin{gathered}
\mathcal{W}^{s-2, s-2}(\mathbb{W}) \\
\mathcal{W}^{s-\frac{1}{2}, s-\frac{1}{2}}(\mathbb{V})
\end{gathered}
$$

and

$$
\boldsymbol{H}_{1}^{s-2}\left(\mathbb{R}^{q}, \mathbb{C}^{\iota(s-2, b+1)}\right):=\begin{gathered}
H^{s-2}\left(\mathbb{R}^{q}, \mathbb{C}^{\iota(s-2, b+1)}\right) \\
\oplus \\
H^{s-\frac{1}{2}}\left(\mathbb{R}^{q}, \mathbb{C}^{\iota\left(s-\frac{1}{2}, b\right)}\right)
\end{gathered} .
$$

Similarly, the operator $\mathcal{A}_{2}=\left(\begin{array}{c}\Delta \\ N\end{array}\right): H^{s}\left(\mathbb{R}_{+}^{n}\right) \longrightarrow \underset{H^{s-\frac{3}{2}}\left(\mathbb{R}^{n-1}\right)}{\longrightarrow}$ can be rephrased as

$$
\mathfrak{A}_{2}^{s}=\left(\begin{array}{cc}
F_{s-2} \Delta E_{s} & F_{s-2} \Delta L_{s} \\
F_{s-\frac{3}{2}}^{\prime} N E_{s} & F_{s-\frac{3}{2}}^{\prime} N L_{s} \\
0 & B_{s-2} \Delta L_{s} \\
0 & B_{s-\frac{3}{2}}^{\prime} N L_{s}
\end{array}\right)
$$

which is continuous as

$$
\mathfrak{A}_{2}^{s}: \begin{gathered}
\mathcal{W}^{s, s}(\mathbb{W}) \\
H^{s}\left(\mathbb{R}^{q}, \mathbb{C}^{\iota(s, b+1)}\right)
\end{gathered} \longrightarrow \quad \begin{gathered}
\mathcal{W}_{2}^{s-2, s-2}(\mathbb{W}) \\
\boldsymbol{H}_{2}^{s-2}\left(\mathbb{R}^{q}, \mathbb{C}^{\iota(s-2, b+1)}\right)
\end{gathered}
$$

where the spaces $\mathcal{W}_{2}^{s-2, s-2}(\mathbb{W})$ and $\boldsymbol{H}_{2}^{s-2}\left(\mathbb{R}^{q}, \mathbb{C}^{\iota(s-2, b+1)}\right)$ are defined by

$$
\begin{array}{ccc}
\mathcal{W}^{s-2, s-2}(\mathbb{W}) & & H^{s-2}\left(\mathbb{R}^{q}, \mathbb{C}^{\iota(s-2, b+1)}\right) \\
\mathcal{W}^{s-\frac{3}{2}, s-\frac{3}{2}}(\mathbb{V}) & \text { and } & \oplus \\
\mathcal{W}^{s-\frac{3}{2}}\left(\mathbb{R}^{q}, \mathbb{C}^{\iota\left(s-\frac{3}{2}, b\right)}\right)
\end{array}
$$

respectively. Let $\mathcal{P}_{1}=\left(\begin{array}{ll}P & K\end{array}\right)$ be a parametrix of $\mathcal{A}_{1}$. Then, as in Section 2.2 we have the operator

$$
R:=N K: H^{s-\frac{1}{2}}\left(\mathbb{R}^{n-1}\right) \longrightarrow H^{s-\frac{3}{2}}\left(\mathbb{R}^{n-1}\right)
$$


as the lower right corner of $\mathcal{A}_{2} \mathcal{P}_{1}$ which is just the Dirichlet-Neumann operator. On the other hand, for the admitted weights, here denoted by $s$, we have the associated edge operator in the lower right corner of $\mathfrak{A}_{2}^{s} \mathfrak{P}_{1}^{s}$. In order to calculate that we first express $\mathfrak{P}_{1}^{s}$, namely

$$
\mathfrak{P}_{1}^{s}=\left(\begin{array}{llll}
F_{s} P E_{s-2} & F_{s} K E_{s-\frac{1}{2}}^{\prime} & F_{s} P L_{s-2} & F_{s} K L_{s-\frac{1}{2}}^{\prime} \\
B_{s} P E_{s-2} & B_{s} K E_{s-\frac{1}{2}}^{\prime} & B_{s} P L_{s-2} & B_{s} K L_{s-\frac{1}{2}}^{\prime}
\end{array}\right) .
$$

Composition with $\mathfrak{A}_{2}^{s}$ gives us

$$
\mathfrak{A}_{2}^{s} \mathfrak{P}_{1}^{s}=\left(\begin{array}{cccc}
1 & 0 & 0 & 0 \\
\Theta_{11} & R_{11} & \Theta_{12} & R_{12} \\
0 & 0 & 1 & 0 \\
\Theta_{21} & R_{21} & \Theta_{22} & R_{22}
\end{array}\right)
$$

where $\Theta_{11}:=F_{s-\frac{3}{2}}^{\prime} N P E_{s-2}, \Theta_{12}:=F_{s-\frac{3}{2}}^{\prime} N P L_{s-2}, \Theta_{21}:=B_{s-\frac{3}{2}}^{\prime} N L_{s} B_{s} P E_{s-2}$, $\Theta_{22}:=B_{s-\frac{3}{2}}^{\prime} N L_{s} B_{s} P L_{s-2}$. Moreover,

$$
\mathfrak{R}^{s}:=\left(R_{i j}\right)_{i, j=1,2}=\left(\begin{array}{cc}
F_{s-\frac{3}{2}}^{\prime} R E_{s-\frac{1}{2}}^{\prime} & F_{s-\frac{3}{2}}^{\prime} R L_{s-\frac{1}{2}}^{\prime} \\
B_{s-\frac{3}{2}}^{\prime} N L_{s} B_{s} K E_{s-\frac{1}{2}}^{\prime} & B_{s-\frac{3}{2}}^{\prime} N L_{s} B_{s} K L_{s-\frac{1}{2}}^{\prime}
\end{array}\right) .
$$

Summing up we have obtained the following result:

Theorem 2.3.2. The (local) Dirichlet-Neumann operator (2.19) has the edge quantisation

$$
\mathfrak{R}^{s}:=\left(R_{i j}\right)_{i, j=1,2}: \begin{array}{cc}
\mathcal{W}^{s-\frac{1}{2}, s-\frac{1}{2}(\mathbb{V})} \\
H^{s-\frac{1}{2}}\left(\mathbb{R}^{q}, \mathbb{C}^{\iota\left(s-\frac{1}{2}, b\right)}\right)
\end{array} \longrightarrow \begin{gathered}
\mathcal{W}^{s-\frac{3}{2}, s-\frac{3}{2}}(\mathbb{V}) \\
H^{s-\frac{3}{2}}\left(\mathbb{R}^{q}, \mathbb{C}^{\iota\left(s-\frac{3}{2}, b\right)}\right)
\end{gathered}
$$

for all $s>\frac{3}{2}, s_{i}-\frac{b}{2} \notin \mathbb{N}$, where $s_{i}:=s-\frac{2 i-1}{2}, i=1,2$.

As in the general case of Section 2.2 the latter theorem also admits a global variant for $\mathbb{V}$ in the meaning of the stretched manifold associated with $\partial X$ with edge $Y$. In other words, applying Theorem 2.2.3 to tha case of the (global) Dirichlet-Neumann operator we obtain a corresponding edge quantisation:

Corollary 2.3.3. For every weight $\gamma \in \mathbb{R}, \gamma>\frac{3}{2}, \gamma_{i}-\frac{b}{2} \notin \mathbb{N}$ for $\gamma_{i}:=\gamma-\frac{2 i-1}{2}, i=$ 1,2 , the Dirichlet-Neumann operator $R$ admits an elliptic operator $\mathfrak{R}^{\gamma}$ in the edge calculus, i.e., a continuous operator

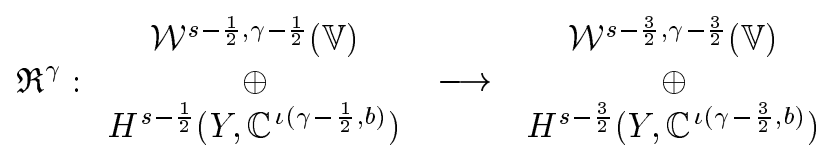

which is Fredholm for every $s \in \mathbb{R}$.

In Theorem 2.4.3 (ii) below we will explicitly express the principal edge symbol of $\mathfrak{R}^{\gamma}$ in local coordinates. 


\subsection{The behaviour of principal edge symbols}

Let us observe the above construction on the level of principal edge symbols. With the reformulation of $\mathcal{A}_{i}$ into $\mathfrak{A}_{i}^{s}$ we can associate the families of operators

$$
\sigma_{\wedge}\left(\mathfrak{A}_{i}^{s}\right)(\eta):=\left(\begin{array}{c}
-|\eta|^{2}+\Delta_{\tilde{x}} \\
\mathrm{r}_{\{t=0\}} \frac{d^{i}-1}{d t^{i-1}}
\end{array}\right): H^{s}\left(\mathbb{R}_{+}^{b+1}\right) \longrightarrow c \begin{gathered}
H^{s-2}\left(\mathbb{R}_{+}^{b+1}\right) \\
\oplus \\
H^{s_{i}}\left(\mathbb{R}^{b}\right)
\end{gathered}
$$

for $s_{i}:=s-\frac{2 i-1}{2}, i=1,2$, and $\eta \in \mathbb{R}^{q} \backslash\{0\}$. Here $\Delta_{\tilde{x}}$ is the Laplace operator in the variables $\tilde{x} \in \mathbb{R}^{b+1}$. By introducing polar coordinates in $\mathbb{R}^{b+1}$ we can write $\Delta_{\tilde{x}}=r^{-2} \sum_{j=0}^{2} a_{j}\left(-r \partial_{r}\right)^{j}$ for coefficients $a_{j} \in \operatorname{Diff}^{2-j}\left(S^{b}\right)$. Moreover, we have the isomorphisms

$$
\tilde{\mathcal{F}}_{s}: H^{s}\left(\mathbb{R}_{+}^{b+1}\right) \longrightarrow \underset{\mathbb{C}^{(s, b+1)}}{\oplus} \text { for } s \geq 0, s-\frac{b+1}{2} \notin \mathbb{N}
$$

$\tilde{\mathcal{F}}_{s}:={ }^{\mathrm{t}}\left(\tilde{F}_{s}, \tilde{B}_{s}\right)$, and

$$
\tilde{\mathcal{F}}_{s_{i}}^{\prime}: H^{s_{i}}\left(\mathbb{R}^{b}\right) \longrightarrow \underset{\mathbb{C}^{\iota\left(s_{i}, b\right)}}{\longrightarrow} \quad \text { for } s \geq 0, s_{i}-\frac{b}{2} \notin \mathbb{N}
$$

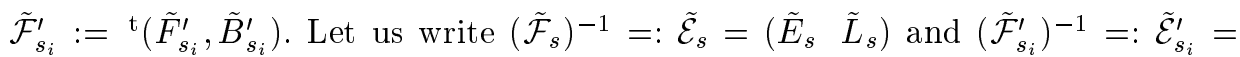
$\left(\begin{array}{ll}\tilde{E}_{s_{i}}^{\prime} & \tilde{L}_{s_{i}}^{\prime}\end{array}\right)$. Then, if we compose (2.20) with the isomorphisms from (2.21), (2.22) we obtain (after rearranging some rows and columns in a suitable manner)

$$
\operatorname{diag}\left(\tilde{\mathcal{F}}_{s-2}, \tilde{\mathcal{F}}_{s_{i}}^{\prime}\right) \sigma_{\wedge}\left(\mathfrak{A}_{i}^{s}\right)(\eta) \tilde{\mathcal{E}}_{s}=\left(\begin{array}{cc}
\tilde{F}_{s-2} A(\eta) \tilde{E}_{s} & \tilde{F}_{s-2} A(\eta) \tilde{L}_{s} \\
\tilde{F}_{s_{i}}^{\prime} T_{i} \tilde{E}_{s} & \tilde{F}_{s_{i}}^{\prime} T_{i} \tilde{L}_{s} \\
0 & \tilde{B}_{s-2} A(\eta) \tilde{L}_{s} \\
0 & \tilde{B}_{s_{i}}^{\prime} T_{i} \tilde{L}_{s}
\end{array}\right)
$$

where $A(\eta):=r^{-2}\left\{-|r \eta|^{2}+\sum_{j=0}^{2} a_{j}\left(-r \partial_{r}\right)^{j}\right\}, T_{i}:=\mathrm{r}_{\{t=0\}} \frac{d^{i-1}}{d t^{i-1}}$,

$$
\operatorname{diag}\left(\tilde{\mathcal{F}}_{s-2}, \tilde{\mathcal{F}}_{s_{i}}^{\prime}\right) \sigma_{\wedge}\left(\mathfrak{A}_{i}^{s}\right)(\eta) \tilde{\mathcal{E}}_{s}: \begin{gathered}
\mathcal{K}^{s, s}\left(\left(S_{+}^{b}\right)^{\wedge}\right) \\
\underset{\mathbb{C}^{\iota(s, b+1)}}{\longrightarrow}
\end{gathered} \longrightarrow \begin{gathered}
\mathcal{K}_{i}^{s-2, s-2}\left(\left(S_{+}^{b}\right)^{\wedge}\right) \\
\mathbb{C}_{i}^{\iota(s-2, b+1)}
\end{gathered}
$$

for

$$
\mathcal{K}_{i}^{s-2, s-2}\left(\left(S_{+}^{b}\right)^{\wedge}\right):=\quad \begin{gathered}
\mathcal{K}^{s-2, s-2}\left(\left(S_{+}^{b}\right)^{\wedge}\right) \\
\mathcal{K}^{s_{i}, s_{i}}\left(\left(S^{b-1}\right)^{\wedge}\right)
\end{gathered}
$$

and $\mathbb{C}_{i}^{\iota(s-2, b+1)}:=\mathbb{C}^{\iota(s-2, b+1)} \oplus \mathbb{C}^{\iota\left(s_{i}, b\right)}$ for $s \geq 0, s_{i}-\frac{b}{2} \notin \mathbb{N}, i=1,2$. 
Proposition 2.4.1. The operator families (2.24) are isomorphisms for all $\eta \neq 0$.

Proof. The ellipticity of $\mathcal{A}_{i}$ as a boundary value problem in the half space entails the bijectivity of the boundary symbols $\sigma_{\partial}\left(\mathcal{A}_{i}\right)\left(\xi^{\prime}\right)$ for every $\xi^{\prime} \neq 0$, cf. the formulas (2.12), (2.14). This implies the bijectivity of (2.20) for every $\eta \neq 0$ and $s>\frac{3}{2}$. In fact, for $\sigma_{\wedge}\left(\mathfrak{A}_{i}^{s}\right)(\eta)$ we have in the notation $(2.20)$

$$
\sigma_{\wedge}\left(\mathfrak{A}_{i}^{s}\right)(\eta)=\operatorname{op}_{\tilde{x}^{\prime}}\left(\sigma_{\partial}\left(\mathcal{A}_{i}\right)\right)(\eta): \mathcal{W}^{s}\left(\mathbb{R}^{b}, H^{s}\left(\mathbb{R}_{+}\right)\right) \longrightarrow \begin{gathered}
\mathcal{W}^{s-2}\left(\mathbb{R}^{b}, H^{s-2}\left(\mathbb{R}_{+}\right)\right) \\
H^{s_{i}}\left(\mathbb{R}^{b}\right)
\end{gathered}
$$

(with the identification $\left.\mathcal{W}^{s}\left(\mathbb{R}^{b}, H^{s}\left(\mathbb{R}_{+}\right)\right)=H^{s}\left(\mathbb{R}_{+}^{b+1}\right)\right), \eta \neq 0$, and we obviously have

$$
\sigma_{\wedge}\left(\mathfrak{A}_{i}^{s}\right)^{-1}(\eta)=\operatorname{op}_{\tilde{x}^{\prime}}\left(\sigma_{\partial}\left(\mathcal{A}_{i}\right)^{-1}\right)(\eta)
$$

Now, as mentioned before, we may apply the isomorphisms (2.21) and (2.22) to the involved spaces and just obtain the opeator families (2.24) as bijections.

Observe that the $(2 \times 1)$ - upper left corners of $(2.24)$, i.e., the corresponding column matrix operators

$$
\sigma_{\wedge}\left(\mathcal{A}_{i}\right)(\eta)=\left(\begin{array}{c}
A(\eta) \\
T_{i}
\end{array}\right): \mathcal{K}^{\tilde{s}, s}\left(\left(S_{+}^{b}\right)^{\wedge}\right) \longrightarrow \mathcal{K}_{i}^{\tilde{s}-2, s-2}\left(\left(S_{+}^{b}\right)^{\wedge}\right)
$$

$\eta \neq 0$, are elliptic boundary value problems for the operator $-|\eta|^{2}+\Delta_{\tilde{x}}$ in the infinite stretched cone $\left(S_{+}^{b}\right)^{\wedge}$ with boundary $\left(S^{b-1}\right)^{\wedge}$. As such they are Fredholm operators for all $\tilde{s}, s>\frac{3}{2}, s_{i}-\frac{b}{2} \notin \mathbb{N}$. In the formulation (2.26) the number $s$ plays the role of the weight which can be treated independently of the smoothness index $\tilde{s}$. As Fredholm operators in the cone calculus the subordinate conormal symbols

$$
\sigma_{M} \sigma_{\wedge}\left(\mathcal{A}_{i}\right)(z)=\left(\begin{array}{c}
\sum_{j=0}^{2} a_{j} z^{j} \\
\mathrm{r}_{\{t=0\}} \frac{d^{i-1}}{d t^{i-1}}
\end{array}\right): H^{\tilde{s}}\left(S_{+}^{b}\right) \longrightarrow \begin{gathered}
H^{\tilde{s}-2}\left(S_{+}^{b}\right) \\
H^{\tilde{s}_{i}}\left(S^{b-1}\right)
\end{gathered}
$$

are bijective for all $\operatorname{Re} z=\frac{b+1}{2}-s$. For those weights $s$ the (differential) cone boundary value problems (2.26) have the index

$$
\text { ind } \sigma_{\wedge}\left(\mathcal{A}_{i}\right)(\eta)=\iota(s-2, b+1)+\iota\left(s_{i}, b\right)-\iota(s, b+1) .
$$

In fact, $\sigma_{\wedge}\left(\mathcal{A}_{i}\right)(\eta)$ is the upper left corner of the isomorphism (2.24) first for $\tilde{s}=s$ and then also for arbitrary $\tilde{s}>\frac{3}{2}$. Then the index of $\sigma_{\wedge}\left(\mathcal{A}_{i}\right)(\eta)$ is necessarily the difference of the dimensions of the finite-dimensional components of the spaces in $(2.24)$. 
Remark 2.4.2. The calculus of boundary value problems on a manifold with conical singularities has been elaborated in [31], [32]. In general, the construction of a pseudo-differential parametrix of an elliptic problem within the corresponding cone algebra is not elementary. We need the 'full' cone calculus with various kinds of quantisations, etc. However, in the present case of operators (2.26) there is an explicit solution: The inverse of the block matrix (2.24) follows by the formula (2.25) and the $(1 \times 2)$-upper left corner of $(2.25)$ is then the parametrix of (2.26).

For purposes below we want to illustrate this for the case $i=1$. In the beginning of this section we calculated $\sigma_{\partial}\left(\mathcal{A}_{1}^{-1}\right)\left(\xi^{\prime}\right)$, cf. the formula $(2.13)$, and we rewrite the first component as op ${ }^{+}\left(l_{+}^{-1}\right)\left(\xi^{\prime}\right) \mathrm{op}^{+}\left(l_{-}^{-1}\right)\left(\xi^{\prime}\right)=\mathrm{op}^{+}\left(-\left|\xi^{\prime}\right|^{2}-\tau^{2}\right)+g\left(\xi^{\prime}\right)$ for some Green operator of type 0 on the half-axis, cf. Theorem 1.2.1.

Then, writing $\xi^{\prime}=\left(\eta, \tilde{\xi}^{\prime}\right)$ it follows that

$$
\sigma_{\wedge}\left(\mathfrak{A}_{1}^{s}\right)^{-1}(\eta)=-\left(\mathrm{op}_{\tilde{x}^{\prime}}(p)(\eta) \mathrm{op}_{\tilde{x}^{\prime}} k\left(\eta, \tilde{\xi}^{\prime}\right)\right),
$$

$\eta \neq 0$ for $p\left(\eta, \tilde{\xi}^{\prime}\right):=\mathrm{op}^{+}\left(|\eta|^{2}+\left|\tilde{\xi}^{\prime}\right|^{2}+\tau^{2}\right)\left(\eta, \tilde{\xi}^{\prime}\right)+g\left(\eta, \tilde{\xi}^{\prime}\right)$.

Theorem 2.4.3. (i) The elliptic cone boundary value problems $(2.26)$ on $\left(S_{+}^{b}\right)^{\wedge}$ for $i=1$ have the family of parametrices

$$
\sigma_{\wedge}\left(\mathcal{A}_{1}\right)^{-1}(\eta)=-\left(\tilde{F}_{s} \mathrm{op}_{\tilde{x}^{\prime}}(p)(\eta) \tilde{E}_{s-2} \quad \tilde{F}_{s} \mathrm{op}_{\tilde{x}^{\prime}}(k)(\eta) \tilde{E}_{s-\frac{1}{2}}^{\prime}\right),
$$

$\eta \neq 0$

(ii) The principal edge symbol of the operator $\mathfrak{R}^{s}$ of Theorem 2.3 .2 has the form

$$
\begin{aligned}
& \sigma_{\wedge}\left(\Re^{s}\right)(\eta)=\left(\begin{array}{ll}
\tilde{F}_{s-\frac{3}{2}}^{\prime} r(\eta) \tilde{E}_{s-\frac{1}{2}}^{\prime} & \tilde{F}_{s-\frac{3}{2}}^{\prime} r(\eta) \tilde{L}_{s-\frac{1}{2}}^{\prime} \\
\tilde{B}_{s-\frac{3}{2}}^{\prime} r(\eta) \tilde{E}_{s-\frac{1}{2}}^{\prime} & \tilde{B}_{s-\frac{3}{2}}^{\prime} r(\eta) \tilde{L}_{s-\frac{1}{2}}^{\prime}
\end{array}\right) \\
& \mathcal{K}^{s-\frac{1}{2}, s-\frac{1}{2}}\left(\left(S^{b-1}\right)^{\wedge}\right) \quad \mathcal{K}^{s-\frac{3}{2}, s-\frac{3}{2}}\left(\left(S^{b-1}\right)^{\wedge}\right) \\
& \begin{array}{cccc}
\left.\mathbb{C}^{\iota\left(s-\frac{1}{2}, b\right)}\right) & \longrightarrow & \oplus \\
\left.\mathbb{C}^{\iota\left(s-\frac{3}{2}, b\right)}\right)
\end{array},
\end{aligned}
$$

$\eta \neq 0$, and is a family of isomorphisms; here $r(\eta):=\mathrm{op}_{\tilde{x}^{\prime}}\left[-\left(|\eta|^{2}+\left|\tilde{\xi}^{\prime}\right|^{2}\right)^{\frac{1}{2}}\right](\eta)$.

Observe that, although the boundary value problems on the infinite cone $\left(S_{+}^{b}\right)^{\wedge}$ belong to the cone algebra, cf. Remark 2.4.2, their Mellin symbolic structure, especially the conormal symbols, are not so explicit in the expressions of the latter theorem. However, there is a general way of producing the Mellin symbols in terms of the actions of operators, similarly as the formulas from the pseudo-differential calculus with the Fourier transform which reproduce the amplitude functions. 


\section{Applications and Remarks}

\subsection{The index of edge symbols in terms of the spectral flow}

We now briefly discuss the question to what extent 'the number' of elliptic edge conditions, i.e., the bundles $J_{ \pm}$in the Fredholm operators (1.32), depend on the weight $\gamma$. We look at the situation of a differential edge boundary value problem of the form (1.12) with an edge-degenerate differential operator $A$ and differential edge-degenerate boundary conditions $T$ such that $\mathcal{A}$ is elliptic in the sense of Definition 2.1.1 (i), (ii).

For simplicity, we content ourselves with the case of trivial bundles $G$ on $\mathbb{V}$, cf. the notation (1.14). Then we may use the notation (1.13) for the weighted Sobolev spaces on $\mathbb{W}$ and $\mathbb{V}$, respectively, and we set

$$
\mathcal{K}^{s-\mu, \gamma-\mu}\left(X^{\wedge}\right):=\mathcal{K}^{s-\mu, \gamma-\mu}\left(X^{\wedge}\right) \oplus \bigoplus_{j=1}^{N} \mathcal{K}^{s-\mu_{j}-\frac{1}{2}, \gamma-\mu_{j}-\frac{1}{2}}\left((\partial X)^{\wedge}\right)
$$

Then, as soon as we find an elliptic operator $\mathfrak{A}^{\gamma} \in \mathfrak{Y}^{\mu}(\mathbb{W} ; \boldsymbol{v})$ for $\boldsymbol{v}=$ $\left(\mathbb{C}, \mathbb{C}^{N} ; J_{-}, J_{+}\right)$with the upper left corner $\mathcal{A}$, the principal edge symbol

$$
\sigma_{\wedge}(\mathcal{A})(y, \eta): \mathcal{K}^{s, \gamma}\left(X^{\wedge}\right) \longrightarrow \mathcal{K}^{s-\mu, \gamma-\mu}\left(X^{\wedge}\right)
$$

is a family of Fredholm operators, parametrised by $(y, \eta) \in T^{*} Y \backslash 0$. For the moment let $\kappa_{\delta}^{(n)}$ denote the action (1.4) on $\mathcal{K}^{s, \gamma}\left(X^{\wedge}\right)$-spaces for $n=\operatorname{dim} X$. By virtue of the homogeneity

$$
\begin{aligned}
\sigma_{\wedge}(\mathcal{A})(y, \delta \eta)= & \operatorname{diag}\left(\delta^{\mu},\left(\delta^{\mu_{j}+\frac{1}{2}}\right)_{j=1, \ldots, N}\right) \cdot \\
& \operatorname{diag}\left(\kappa_{\delta}^{(n)}, \operatorname{diag}\left(\kappa_{\delta}^{n-1}\right)_{j=1, \ldots, N}\right) \sigma_{\wedge}(\mathcal{A})(y, \eta)\left(\kappa_{\delta}^{(n)}\right)^{-1}
\end{aligned}
$$

for all $\delta \in \mathbb{R}_{+},(y, \eta) \in T^{*} Y \backslash 0$, we can interpret (3.1) as a family of Fredholm operators on the compact topological space $S^{*} Y$. There is then a $K$ - theoretic index element

$$
\operatorname{ind}_{S * Y} \sigma_{\wedge}(\mathcal{A}) \in K\left(S^{*} Y\right) .
$$

Then the fact that $\sigma_{\wedge}(\mathcal{A})$ completes to a family of isomorphisms (1.33) has the consequence that the index element is equal to $\left[\pi_{1}^{*} J_{+}\right]-\left[\pi_{1}^{*} J_{-}\right]$, i.e.,

$$
\operatorname{ind}_{S * Y} \sigma_{\wedge}(\mathcal{A}) \in \pi_{1}^{*} K(Y) \text {, }
$$

where $\pi_{1}: S^{*} Y \longrightarrow Y,(y, \eta) \rightarrow y$, is the canonical projection.

Recall the general fact that (3.2) is a necessary and sufficient condition for the existence of extra edge conditions of trace and potential type that complete $\mathcal{A}$ to a Fredholm operator $\mathfrak{A}^{\gamma}$. This is an analogue of the topological obstruction for the 
existence of elliptic boundary value problems to an elliptic operator $A$, cf. [1]. It may happen that there is another weight $\beta \in \mathbb{R}$ such that also

$$
\sigma_{\wedge}(\mathcal{A})(y, \eta): \mathcal{K}^{s, \beta}\left(X^{\wedge}\right) \longrightarrow \mathcal{K}^{s-\mu, \beta-\mu}\left(X^{\wedge}\right)
$$

is a family of Fredholm operators. In order to characterise such $\beta$ we look at the set $D(y)$ of all $z \in \mathbb{C}$ such that

$$
\sigma_{M} \sigma_{\wedge}(\mathcal{A})(y, z): H^{s}(X) \longrightarrow \boldsymbol{H}^{s-\mu}(X)
$$

is not a bijective operator for any sufficiently large real $s$; here $\boldsymbol{H}^{s-\mu}(X):=$ $H^{s-\mu}(X) \oplus \bigoplus_{j=1}^{N} H^{s-\mu_{j}-\frac{1}{2}}(\partial X)$. Then (3.3) is Fredholm whenever $D(y) \cap$ $\Gamma_{\frac{b+1}{2}-\beta}=\emptyset$ (and arbitrary $\eta \neq 0$ ). If (3.3) is Fredholm for all $y \in Y$ we can ask again the nature of extra edge conditions.

Denoting for the moment the Fredholm family (3.3) by $\sigma_{\wedge}\left(\mathcal{A}^{\beta}\right)$, we have the following result:

Theorem 3.1.1. The property $\operatorname{ind}_{S^{*} Y} \sigma_{\wedge}\left(\mathcal{A}^{\beta}\right) \in \pi_{1}^{*} K(Y)$ is independent of the choice of $\beta$.

A proof may be found in [19].

It is now an interesting task to really calculate $\operatorname{ind}_{S^{*} Y} \sigma_{\wedge}\left(\mathcal{A}^{\beta}\right)$ in terms of $\operatorname{ind}_{S^{*} Y} \sigma_{\wedge}\left(\mathcal{A}^{\gamma}\right)$; the latter is assumed to be known. The general answer is determined by the generalised spectral flow, cf. [24], associated with the conormal symbol $\sigma_{M} \sigma_{\wedge}(\mathcal{A})(y, z)$, cf. (1.34), combined with a cutting and pasting construction for elliptic families as a generalisation of the results of [27].

In order to formulate the result we first recall that for every fixed $y \in Y$ we have

$$
\text { ind } \sigma_{\wedge}\left(\mathcal{A}^{\beta}\right)(y, \eta)-\operatorname{ind} \sigma_{\wedge}\left(\mathcal{A}^{\gamma}\right)(y, \eta)=\boldsymbol{n}(\gamma, \beta)
$$

where $\boldsymbol{n}(\gamma, \beta)$ is the sum of null-multiplicities (in the sense of Gohberg and $\mathrm{Si}$ gal [10]) of the non-bijectivity points of $\sigma_{M} \sigma_{\wedge}(\mathcal{A})(y, z)$ in the strip $\frac{b+1}{2}-\gamma<$ $\operatorname{Re} z<\frac{b+1}{2}-\beta$, cf. [19, Proposition 4.1.2]. The number (3.5) can also be interpreted as the spectral flow $\operatorname{sf}_{(\gamma, \beta)}$ of the family of conormal symbols

$$
\sigma_{M} \sigma_{\wedge}(\mathcal{A})(y, \tau, t):=\sigma_{M} \sigma_{\wedge}(\mathcal{A})\left(y, \frac{b+1}{2}-(1-t) \gamma-t \beta+i \tau\right)
$$

for $0 \leq t \leq 1$ and fixed $y$, cf. [24], i.e., we have

$$
\operatorname{sf}_{(\gamma, \beta)} \sigma_{M} \sigma_{\wedge}(\mathcal{A})=\boldsymbol{n}(\gamma, \beta)
$$

(in this notation $\gamma$ and $\beta$ are fixed). However, the information of [24] is more precise; it refers to families of conormal symbols, parametrised by the topological space $Y$. In other words from [24] we have the first assertion of the following theorem: 
Theorem 3.1.2. Let $\mathcal{A}$ be a differential edge boundary value problem of the form (1.12) that is elliptic in the sense of Definition 2.1.1 (i), (ii). Assume that the relation (3.2) is satisfied.

(i) The (generalised) spectral flow belonging to the conormal symbol (1.34) in the strip $\frac{b+1}{2}-\gamma<\operatorname{Re} z<\frac{b+1}{2}-\beta$ defines an element

$$
\operatorname{sf}_{(\gamma, \beta)} \sigma_{M} \sigma_{\wedge}(\mathcal{A}) \in K(Y) .
$$

(ii) We have

$$
\operatorname{ind}_{Y} \sigma_{\wedge}\left(\mathcal{A}^{\gamma}\right)-\operatorname{ind}_{Y} \sigma_{\wedge}\left(\mathcal{A}^{\beta}\right)=\operatorname{sf}_{(\gamma, \beta)} \sigma_{M} \sigma_{\wedge}(\mathcal{A})
$$

Proof. The operator $\mathcal{A}$ near the edge $Y$ has the form $\mathcal{A}=\left(\begin{array}{c}A \\ T\end{array}\right)$ for

$$
A=r^{-\mu} \sum_{j+|\alpha| \leqq \mu} a_{j \alpha}(r, y)\left(-r \partial_{r}\right)^{j}\left(r D_{y}\right)^{\alpha}
$$

with coefficients $a_{j \alpha}(r, y) \in C^{\infty}\left(\overline{\mathbb{R}}_{+} \times Y, \operatorname{Diff}^{\mu-(j+|\alpha|)}(X)\right)$ and $T={ }^{\mathrm{t}}\left(T_{1}, \ldots, T_{N}\right)$, $T_{j}=\mathrm{r}_{\partial X \times Y} B_{j}$, for $B_{j}=r^{-\mu_{j}} \sum_{k+|\beta| \leqq \mu_{j}} b_{j, k \beta}(r, y)\left(-r \partial_{r}\right)^{k}\left(r D_{y}\right)^{\beta}$ with $b_{j, k \beta}(r, y) \in C^{\infty}\left(\overline{\mathbb{R}}_{+} \times Y\right.$, Diff $\left.\mu_{j}-(k+|\beta|)\left(U^{\wedge}\right)\right)$. Then we have

$$
\begin{aligned}
\sigma_{\wedge}\left(\mathcal{A}^{\gamma}\right)(y, \eta)= & \operatorname{diag}\left(r^{-\mu},\left(r^{-\mu_{j}}\right)_{j=1, \ldots, N}\right) \cdot \\
& \left(\begin{array}{c}
\sum_{j+|\alpha| \leqq \mu} a_{j \alpha}(0, y)\left(-r \partial_{r}\right)^{j}(r \eta)^{\alpha} \\
\left(\mathrm{r}_{\partial X \times Y} \sum_{k+|\beta| \leqq \mu_{j}} b_{j, k \beta}(0, y)\left(-r \partial_{r}\right)^{k}(r \eta)^{\beta}\right)_{j=1, \ldots, N}
\end{array}\right),
\end{aligned}
$$

which is the Fredhlom family (3.1). Its principal conormal symbol has the form

$$
a(y, z)=\left(\begin{array}{c}
\sum_{k=0}^{\mu} a_{k 0}(0, y) z^{k} \\
\left(\mathrm{r}_{\partial X} \sum_{k=0}^{\mu} b_{j, k 0}(0, y) z^{k}\right)_{j=1, \ldots, N}
\end{array}\right)
$$

which is a family of isomorphisms parametrised by $(y, z) \in Y \times \Gamma_{\frac{b+1}{2}-\gamma}$.

Let us set

$$
K^{\gamma}:=\operatorname{diag}\left(r^{\mu},\left(r^{\mu_{j}}\right)_{j=1, \ldots, N}\right) \sigma_{\wedge}\left(\mathcal{A}^{\gamma}\right)(y, \eta)
$$

and

$$
\mathcal{K}^{s, \gamma}:=\mathcal{K}^{s, \gamma}\left(X^{\wedge}\right), \tilde{\mathcal{K}}^{s-\mu, \gamma}:=\operatorname{diag}\left(r^{\mu},\left(r^{\mu_{j}}\right)_{j=1, \ldots, N}\right) \mathcal{K}^{s-\mu, \gamma-\mu}\left(X^{\wedge}\right)
$$

In a similar manner, if $\beta \in \mathbb{R}$ is another weight such that $\sigma_{M} \sigma_{\wedge}\left(\mathcal{A}^{\gamma}\right)(y, z)$ defines a family of isomorphisms (3.4) for all $(y, z) \in Y \times \Gamma_{\frac{b+1}{2}-\beta}$, we form the operator $K^{\beta}$ and spaces $\mathcal{K}^{s, \beta}$ and $\tilde{\mathcal{K}}^{s-\mu, \beta}$. Thus we have two families

$$
K^{\gamma}: \mathcal{K}^{s, \gamma} \longrightarrow \tilde{\mathcal{K}}^{s-\mu, \gamma}, K^{\beta}: \mathcal{K}^{s, \beta} \longrightarrow \tilde{\mathcal{K}}^{s-\mu, \beta}
$$


of Fredholm operators. We then have

$$
\operatorname{ind}_{Y} K^{\gamma}=\operatorname{ind}_{Y} \sigma_{\wedge}\left(\mathcal{A}^{\gamma}\right), \operatorname{ind}_{Y} K^{\beta}=\operatorname{ind}_{Y} \sigma_{\wedge}\left(\mathcal{A}^{\beta}\right) .
$$

We want to compare (3.7) with modified families

$$
B^{\gamma}: \mathcal{H}^{s,(\gamma, \delta)} \longrightarrow \tilde{\mathcal{H}}^{s-\mu,(\gamma, \delta)}, B^{\beta}: \mathcal{H}^{s,(\beta, \delta)} \longrightarrow \tilde{\mathcal{H}}^{s-\mu,(\beta, \delta)}
$$

with the following meaning:

$$
B^{\gamma}:=\mathrm{op}_{M}^{\gamma-\frac{b}{2}}(a)+\omega c, B^{\beta}:=\mathrm{op}_{M}^{\beta-\frac{b}{2}}(a)+\omega c
$$

for

$$
c(y, z):=\left(\begin{array}{c}
\sum_{\substack{k+|\alpha| \leq \mu \\
|\alpha|>0}} a_{k \alpha}(0, y)\left(-r \partial_{r}\right)^{j}(r \eta)^{\alpha} \\
\left(\mathrm{r}_{(\partial X)^{\wedge}} \sum_{\substack{k+|\alpha| \leqq \mu_{j} \\
|\alpha|>0}} b_{j, k \alpha}(0, y)\left(-r \partial_{r}\right)^{k}(r \eta)^{\alpha}\right)_{j=1, \ldots, N}
\end{array}\right)
$$

where $\omega(r)$ is any fixed cut-off function on the half-axis, and

$$
\begin{aligned}
\mathcal{H}^{s,(\gamma, \delta)} & :=\omega_{1} \mathcal{H}^{s, \gamma}\left(X^{\wedge}\right)+\left(1-\omega_{1}\right) \mathcal{H}^{s, \delta}\left(X^{\wedge}\right), \\
\tilde{\mathcal{H}}^{s-\mu,(\gamma, \delta)} & :=\omega_{1}\left(\begin{array}{c}
\mathcal{H}^{s-\mu, \gamma}\left(X^{\wedge}\right) \\
\oplus \\
\oplus_{j=1}^{N} \mathcal{H}^{s-\mu_{j}-\frac{1}{2}, \gamma-\frac{1}{2}}\left((\partial X)^{\wedge}\right)
\end{array}\right) \\
& +\left(1-\omega_{1}\right)\left(\begin{array}{c}
\mathcal{H}^{s-\mu, \delta}\left(X^{\wedge}\right) \\
\oplus \\
\oplus_{j=1}^{N} \mathcal{H}^{s-\mu_{j}-\frac{1}{2}, \delta-\frac{1}{2}}\left((\partial X)^{\wedge}\right)
\end{array}\right)
\end{aligned}
$$

for any other cut-off function $\omega_{1}(r)$, with a weight $\delta \geqq \max (\gamma, \beta)$; in a similar manner we form the spaces $\mathcal{H}^{s,(\beta, \delta)}$ and $\tilde{\mathcal{H}}^{s-\mu,(\beta, \delta)}$, respectively. We fix $\delta$ in such a way that (3.6) defines also a bijection (3.4) for all $(y, z) \in Y \times \Gamma_{\frac{b+1}{2}-\delta}$. Because we may take $\delta=\gamma$ this is no additional assumption. The operators (3.9) are defined on the spaces with double weights in a similar manner as in [12], see also [37]. In this situation we know the pointwise relation

$$
\text { ind } B^{\beta}-\text { ind } B^{\gamma}=\boldsymbol{n}(\gamma, \beta) \text {. }
$$

We know, in fact, more, namely,

$$
\operatorname{ind}_{Y} B^{\beta}-\operatorname{ind}_{Y} B^{\gamma}=\operatorname{sf}_{(\gamma, \beta)} \sigma_{M} \sigma_{\wedge}(\mathcal{A}) .
$$

This follows from the relations $\operatorname{ind}_{Y} B^{\beta}=\operatorname{ind}_{Y} \operatorname{op}_{M}^{\beta-\frac{b}{2}}(a), \operatorname{ind}_{Y} B^{\gamma}=$ $\operatorname{ind}_{Y} \operatorname{op}_{M}^{\gamma-\frac{b}{2}}(a)$, since the operator family $\omega c$ in (3.10) takes values in compact operators, and

$$
\operatorname{ind}_{Y} \operatorname{op}_{M}^{\beta-\frac{b}{2}}(a)=\operatorname{sf}_{(\delta, \beta)} \sigma_{M} \sigma_{\wedge}(\mathcal{A}), \operatorname{ind}_{Y} \operatorname{op}_{M}^{\gamma-\frac{b}{2}}(a)=\operatorname{sf}_{(\delta, \gamma)} \sigma_{M} \sigma_{\wedge}(\mathcal{A}),
$$


together with the relation $\operatorname{sf}_{(\delta, \beta)} \sigma_{M} \sigma_{\wedge}(\mathcal{A})-\operatorname{sf}_{(\delta, \gamma)} \sigma_{M} \sigma_{\wedge}(\mathcal{A})=\operatorname{sf}_{(\gamma, \beta)} \sigma_{M} \sigma_{\wedge}(\mathcal{A})$, cf. [24]. On the other hand from the results of [26] we know that

$$
\operatorname{ind}_{Y} K^{\beta}-\operatorname{ind}_{Y} K^{\gamma}=\operatorname{ind}_{Y} B^{\beta}-\operatorname{ind}_{Y} B^{\gamma} .
$$

The relation (3.12) follows in a similar manner as in [27], now for families. The assumptions are that for some sufficiently large $R>0$, such that $\omega \equiv 1$ on $[0, R]$, we have

$$
\begin{gathered}
\left.K^{\gamma}\right|_{0<r<R}=\left.B^{\gamma}\right|_{0<r<R},\left.K^{\beta}\right|_{0<r<R}=\left.B^{\beta}\right|_{0<r<R}, \\
\left.K^{\gamma}\right|_{R<r<\infty}=\left.K^{\beta}\right|_{R<r<\infty},\left.B^{\gamma}\right|_{R<r<\infty}=\left.B^{\beta}\right|_{R<r<\infty}
\end{gathered}
$$

(modulo compact operators in the respective spaces).

Then (3.8), (3.11) and (3.12) yield the assertion (ii) of the theorem.

Remark 3.1.3. There is an analogue of Theorem 3.1.2 also for elliptic pseudodifferential edge problems in the sense of Definition 2.1.1. In particular, we may have elliptic operators on $\mathbb{V}$ as a 'closed' manifold with edges. Applying this, for instance, to the operator $\mathcal{R}$ in the upper left corner of (2.5) we obtain

$$
\operatorname{ind}_{Y} \sigma_{\wedge}\left(\mathcal{R}^{\gamma}\right)-\operatorname{ind}_{Y} \sigma_{\wedge}\left(\mathcal{R}^{\beta}\right)=\operatorname{sf}_{(\gamma, \beta)} \sigma_{M} \sigma_{\wedge}(\mathcal{R}) .
$$

\subsection{Edge quantisation for arbitrary weights}

We now draw some conclusions from the results of the preceding section for the edge quantised operators from Section 2.2 .

Let us first return to Theorems 2.1.3 and 2.1.4 and observe that there are analogues on the level of principal edge symbols of the corresponding $2 \times 2$-upper left corners of the operators. Let us only consider the Theorem 2.1.4; (the case of Theorem 2.1.3 is left to the reader).

Let $\mathcal{A}_{j}, j=1,2$ denote the $2 \times 2$-upper left corner of $\mathfrak{A}_{j}$,

$$
\mathcal{A}_{j}: \mathcal{W}^{s, \gamma}\left(\mathbb{W} ; G_{-}^{j}\right) \longrightarrow \mathcal{W}^{s-\mu, \gamma-\mu}\left(\mathbb{W} ; G_{+}^{j}\right)
$$

(which are, of course, not necessarily Fredholm) and

$$
\sigma_{\wedge}\left(\mathcal{A}_{j}\right)(y, \eta): \mathcal{K}^{s, \gamma}\left(X^{\wedge} ; G_{-}^{j}\right) \longrightarrow \mathcal{K}^{s-\mu, \gamma-\mu}\left(X^{\wedge} ; G_{+}^{j}\right)
$$

the corresponding homogeneous principal edge symbols which are families of Fredholm operators, parametrised by $T^{*} Y \backslash 0$, cf. the formula (1.27). We will restrict them to the unit cosphere bundle $S^{*} Y$. Then, using operators $\tilde{\mathcal{A}}_{j}$ in a similar sense as in Section 2.1 and the corresponding parametrices $\mathcal{P}_{j}$ and $\tilde{\mathcal{P}}_{j}$, respectively, we have

$$
\operatorname{ind}_{Y} \sigma_{\wedge}\left(\tilde{\mathcal{A}}_{2} \tilde{\mathcal{P}}_{1}\right)=\operatorname{ind}_{Y} \sigma_{\wedge}\left(\mathcal{A}_{2}\right)-\operatorname{ind}_{Y} \sigma_{\wedge}\left(\mathcal{A}_{1}\right)=\operatorname{ind}_{Y} \sigma_{\wedge}(\mathcal{R})
$$


where $\mathcal{R}$ denotes the upper left corner of $\Re$.

Let us now consider the operators $\mathcal{A}_{j}$ and the associated $\tilde{\mathcal{A}}_{j}$ for two weights $\gamma$ and $\beta$, denoted by $\mathcal{A}_{j}^{\gamma}, \tilde{\mathcal{A}}_{j}^{\gamma}$ and $\mathcal{A}_{j}^{\beta}, \tilde{\mathcal{A}}_{j}^{\beta}$, respectively, $j=1,2$. Let $\mathcal{R}^{\gamma}$ and $\mathcal{R}^{\beta}$ denote the upper left corners of (2.5), realised for $\gamma$ and $\beta$, respectively. Then applying (3.14) for the weights $\gamma$ and $\beta$ it follows that

$$
\begin{aligned}
& \operatorname{ind}_{Y} \sigma_{\wedge}\left(\tilde{\mathcal{A}}_{2}^{\gamma} \tilde{\mathcal{P}}_{1}^{\gamma}\right)=\operatorname{ind}_{Y} \sigma_{\wedge}\left(\mathcal{A}_{2}^{\gamma}\right)-\operatorname{ind}_{Y} \sigma_{\wedge}\left(\mathcal{A}_{1}^{\gamma}\right)=\operatorname{ind}_{Y} \sigma_{\wedge}\left(\mathcal{R}^{\gamma}\right), \\
& \operatorname{ind}_{Y} \sigma_{\wedge}\left(\tilde{\mathcal{A}}_{2}^{\beta} \tilde{\mathcal{P}}_{1}^{\beta}\right)=\operatorname{ind}_{Y} \sigma_{\wedge}\left(\mathcal{A}_{2}^{\beta}\right)-\operatorname{ind}_{Y} \sigma_{\wedge}\left(\mathcal{A}_{1}^{\beta}\right)=\operatorname{ind}_{Y} \sigma_{\wedge}\left(\mathcal{R}^{\beta}\right) .
\end{aligned}
$$

This gives us

$$
\begin{aligned}
{\left[\operatorname{ind}_{Y} \sigma_{\wedge}\left(\mathcal{A}_{2}^{\gamma}\right)-\operatorname{ind}_{Y} \sigma_{\wedge}\left(\mathcal{A}_{1}^{\gamma}\right)\right] } & -\left[\operatorname{ind}_{Y} \sigma_{\wedge}\left(\mathcal{A}_{2}^{\beta}\right)-\operatorname{ind}_{Y} \sigma_{\wedge}\left(\mathcal{A}_{1}^{\beta}\right)\right] \\
& =\operatorname{ind}_{Y} \sigma_{\wedge}\left(\mathcal{R}^{\gamma}\right)-\operatorname{ind}_{Y} \sigma_{\wedge}\left(\mathcal{R}^{\beta}\right) .
\end{aligned}
$$

Moreover, Remark 3.1.3, applied to the pseudo-differential edge problems $\mathcal{A}_{j}^{\gamma}$ and $\mathcal{A}_{j}^{\beta}$ gives us $\operatorname{ind}_{Y} \sigma_{\wedge}\left(\mathcal{A}_{j}^{\gamma}\right)-\operatorname{ind}_{Y} \sigma_{\wedge}\left(\mathcal{A}_{j}^{\beta}\right)=\operatorname{sf}_{(\gamma, \beta)} \sigma_{M} \sigma_{\wedge}\left(\mathcal{A}_{j}\right)$ for $j=1,2$, and the relation (3.13) yields the following result:

Theorem 3.2.1. We have

$$
\operatorname{sf}_{(\gamma, \beta)} \sigma_{M} \sigma_{\wedge}\left(\mathcal{A}_{2}\right)-\operatorname{sf}_{(\gamma, \beta)} \sigma_{M} \sigma_{\wedge}\left(\mathcal{A}_{1}\right)=\operatorname{sf}_{(\gamma, \beta)} \sigma_{M} \sigma_{\wedge}(\mathcal{R}) .
$$

These considerations allow us to edge quantise the operators reduced to $\partial X$ with respect to the edge $Y$ not only for large weights $\gamma$ but for arbitrary 'bad' weights $\beta \leq \gamma$. In fact, Theorem 2.2.3 gives us edge quantisations for weights $\gamma$ as in Theorem 2.2.1 and, at the same time, an explicit expression for the index of the principal symbol of the upper left corner $\mathcal{R}^{\gamma}$ of $\mathfrak{R}^{\gamma}$, namely,

$$
\sum_{j=1}^{m}\left\{\iota\left(\gamma-\mu_{2, j}-\frac{1}{2}, b\right)-\iota\left(\gamma-\mu_{1, j}-\frac{1}{2}, b\right)\right\},
$$

which is a consequence of the formula (2.11) together with the relation (3.15). Now, as soon we know a weight $\beta \leq \gamma$ for which $\sigma_{M} \sigma_{\wedge}(\mathcal{R})(y, z)$ is holomorphic in a neighbourhood of $\Gamma_{\frac{b+1}{2}-\beta}$ for all $y \in Y$ (which is the case when both $\sigma_{M} \sigma_{\wedge}\left(\mathcal{A}_{1}\right)(y, z)$ and $\sigma_{M} \sigma_{\wedge}\left(\mathcal{A}_{2}\right)(y, z)$ are holomorphic near that line) then the relation (3.13) allows us to pass to elliptic operators

$$
\begin{aligned}
& \mathfrak{R}^{\beta}: \begin{array}{clc}
\oplus_{j=1}^{m} \mathcal{W}^{s-\mu_{1, j}-\frac{1}{2}, \beta-\mu_{1, j}-\frac{1}{2}}(\mathbb{V}) & & \oplus_{j=1}^{m} \mathcal{W}^{s-\mu_{2, j}-\frac{1}{2}, \beta-\mu_{2, j}-\frac{1}{2}}(\mathbb{V}) \\
\oplus & \longrightarrow & \oplus
\end{array} \\
& H^{s}\left(Y, J_{-}\right) \quad H^{s}\left(Y, J_{+}\right)
\end{aligned}
$$

in the edge calculus, for bundles $J_{-}, J_{+} \in \operatorname{Vect}(Y)$ such that

$$
\left[J_{+}\right]-\left[J_{-}\right]=\left[\sum_{j=1}^{m} \iota\left(\gamma-\mu_{2, j}-\frac{1}{2}, b\right)\right]-\left[\sum_{j=1}^{m} \iota\left(\gamma-\mu_{1, j}-\frac{1}{2}, b\right)\right]-\operatorname{sf}_{(\gamma, \beta)} \sigma_{M} \sigma_{\wedge}(\mathcal{R}) .
$$


The construction of additional conditions to an upper left corner $\mathcal{R}^{\beta}$ with suitable such bundles $J_{ \pm}$is a general procedure which we do not carry out here explicitly; for similar constructions, see [13] and [19, Theorem 4.2.2].

\subsection{Appendix: Proof of Theorem 2.4.3}

Proof. (i) Let us first compute the inverse of the block matrix (2.24) for $i=1$. This is equal to $\tilde{\mathcal{F}}_{s} \sigma_{\wedge}\left(\mathfrak{A}_{1}^{s}\right)^{-1}(\eta) \operatorname{diag}\left(\tilde{\mathcal{E}}_{s-2}, \tilde{\mathcal{E}}_{s_{1}}^{\prime}\right)$, which, after using $(2.27)$ becomes

$$
\left(\begin{array}{c}
\tilde{F}_{s} \\
\tilde{B}_{s}
\end{array}\right)\left[-\left(\operatorname{op}_{\tilde{x}^{\prime}}(p)(\eta) \mathrm{op}_{\tilde{x}^{\prime}} k\left(\eta, \tilde{\xi}^{\prime}\right)\right)\right]\left(\begin{array}{cccc}
\tilde{E}_{s-2} & 0 & \tilde{L}_{s-2} & 0 \\
0 & \tilde{E}_{s-\frac{1}{2}}^{\prime} & 0 & \tilde{L}_{s-\frac{1}{2}}^{\prime}
\end{array}\right)
$$

for $p\left(\eta, \tilde{\xi}^{\prime}\right):=\mathrm{op}^{+}\left(|\eta|^{2}+\left|\tilde{\xi}^{\prime}\right|^{2}+\tau^{2}\right)\left(\eta, \tilde{\xi}^{\prime}\right)+g\left(\eta, \tilde{\xi}^{\prime}\right)$.

The $(1 \times 2)$-upper left corner of $(3.17)$ is $-\left(\tilde{F}_{s} \mathrm{op}_{\tilde{x}^{\prime}}(p)(\eta) \tilde{E}_{s-2} \quad \tilde{F}_{s} \mathrm{op}_{\tilde{x}^{\prime}}(k)(\eta) \tilde{E}_{s-\frac{1}{2}}^{\prime}\right)$, which according to the Remark 2.4.2 is exactly the parametrix of $(2.26)$ on $\left(S_{+}^{b}\right)^{\wedge}$ for $i=1$, i.e.,

$$
\sigma_{\wedge}\left(\mathcal{A}_{1}\right)^{-1}(\eta)=-\left(\tilde{F}_{s} \mathrm{op}_{\tilde{x}^{\prime}}(p)(\eta) \tilde{E}_{s-2} \quad \tilde{F}_{s} \mathrm{op}_{\tilde{x}^{\prime}}(k)(\eta) \tilde{E}_{s-\frac{1}{2}}^{\prime}\right) .
$$

(ii) In order to calculate the principal edge symbol of the edge quantised operator $\mathfrak{R}^{s}$ we will proceed as we did for the Agranovich-Dynin theorem; we will multiply the extended operator of $\sigma_{\wedge}\left(\mathfrak{A}_{2}^{s}\right)(\eta)$ with the previous inverse of the extended operator of $\sigma_{\wedge}\left(\mathfrak{A}_{1}^{s}\right)(\eta),(3.17)$. This gives us

$$
\begin{aligned}
& \operatorname{diag}\left(\tilde{\mathcal{F}}_{s-2}, \tilde{\mathcal{F}}_{s_{2}}^{\prime}\right) \sigma_{\wedge}\left(\mathfrak{A}_{2}^{s}\right)(\eta) \tilde{\mathcal{E}}_{s} \tilde{\mathcal{F}}_{s} \sigma_{\wedge}\left(\mathfrak{A}_{1}^{s}\right)^{-1}(\eta) \operatorname{diag}\left(\tilde{\mathcal{E}}_{s-2}, \tilde{\mathcal{E}}_{s_{1}}^{\prime}\right) \\
= & \operatorname{diag}\left(\tilde{\mathcal{F}}_{s-2}, \tilde{\mathcal{F}}_{s_{2}}^{\prime}\right) \sigma_{\wedge}\left(\mathfrak{A}_{2}^{s}\right)(\eta) \sigma_{\wedge}\left(\mathfrak{A}_{1}^{s}\right)^{-1}(\eta) \operatorname{diag}\left(\tilde{\mathcal{E}}_{s-2}, \tilde{\mathcal{E}}_{s_{1}}^{\prime}\right) .
\end{aligned}
$$

The term in the middle of (3.18) follows by the formula (2.25), i.e.,

$$
\sigma_{\wedge}\left(\mathfrak{A}_{2}^{s}\right)(\eta) \sigma_{\wedge}\left(\mathfrak{A}_{1}^{s}\right)^{-1}(\eta)=\mathrm{op}_{\tilde{x}^{\prime}}\left(\sigma_{\partial}\left(\mathcal{A}_{2}\right) \sigma_{\partial}\left(\mathcal{A}_{1}\right)^{-1}\right)(\eta),
$$

which is equal to

$$
\mathrm{op}_{\tilde{x}^{\prime}}\left(\begin{array}{cc}
1 & 0 \\
h\left(\eta, \tilde{\xi}^{\prime}\right) & -\left(|\eta|^{2}+\left|\tilde{\xi}^{\prime}\right|^{2}\right)^{\frac{1}{2}}
\end{array}\right)
$$

for $h\left(\eta, \tilde{\xi}^{\prime}\right)=\mathrm{r}_{\{t=0\}} \frac{d}{d t} p\left(\eta, \tilde{\xi}^{\prime}\right)$, a trace boundary symbol of type 0 . If we denote $H(\eta):=\mathrm{op}_{\tilde{x}^{\prime}}\left(h\left(\eta, \tilde{\xi}^{\prime}\right)\right)$ and $r(\eta):=\mathrm{op}_{\tilde{x}^{\prime}}\left[-\left(|\eta|^{2}+\left|\tilde{\xi}^{\prime}\right|^{2}\right)^{\frac{1}{2}}\right](\eta)$ we obtain

$$
\sigma_{\wedge}\left(\mathfrak{A}_{2}^{s}\right)(\eta) \sigma_{\wedge}\left(\mathfrak{A}_{1}^{s}\right)^{-1}(\eta)=\left(\begin{array}{cc}
1 & 0 \\
H(\eta) & r(\eta)
\end{array}\right) .
$$

Now, (3.18) becomes

$$
\left(\begin{array}{ccccc}
\tilde{F}_{s-\frac{3}{2}}^{\prime} H(\eta) \tilde{E}_{s-2} & \tilde{F}_{s-\frac{3}{2}}^{\prime} r(\eta) \tilde{E}_{s-\frac{1}{2}}^{\prime} & \tilde{F}_{s-\frac{3}{2}}^{\prime} H(\eta) \tilde{L}_{s-2} & \tilde{F}_{s-\frac{3}{2}}^{\prime} r(\eta) \tilde{L}_{s-\frac{1}{2}}^{\prime} \\
0 & 0 & 1 & 0 \\
\tilde{B}_{s-\frac{3}{2}}^{\prime} H(\eta) \tilde{E}_{s-2} & \tilde{B}_{s-\frac{3}{2}}^{\prime} r(\eta) \tilde{E}_{s-\frac{1}{2}}^{\prime} & \tilde{B}_{s-\frac{3}{2}}^{\prime} H(\eta) \tilde{L}_{s-2} & \tilde{B}_{s-\frac{3}{2}}^{\prime} r(\eta) \tilde{L}_{s-\frac{1}{2}}^{\prime}
\end{array}\right)
$$


which gives us the principal edge symbol of the edge quantised operator $\mathfrak{R}^{s}$ of Theorem 2.3.2 (ii), namely $\sigma_{\wedge}\left(\mathfrak{R}^{s}\right)(\eta)=\tilde{\mathcal{F}}_{s_{2}}^{\prime} r(\eta) \tilde{\mathcal{E}}_{s_{1}}^{\prime}$ having the required mapping properties, cf. (2.21) and (2.22).

\section{References}

[1] M.F. Atiyah and R. Bott, The index problem for manifolds with boundary, Coll. Differential Analysis, Tata Institute Bombay, Oxford University Press, Oxford, 1964, pp. 175-186.

[2] L. Boutet de Monvel, Boundary problems for pseudo-differential operators, Acta Math. 126 (1971), 11-51.

[3] N. Dines, G. Harutjunjan, and B.-W. Schulze, The Zaremba problem in edge Sobolev spaces, Preprint 2003/13, Institut für Mathematik, Potsdam, 2003.

[4] N. Dines and B.-W. Schulze, Mellin-edge-representations of elliptic operators, Preprint 2003/18, Institut für Mathematik, Potsdam, 2003, Math. Meth. in the Appl. Sci.(to appear).

[5] Ju. V. Egorov and B.-W. Schulze, Pseudo-differential operators, singularities, applications, Operator Theory, Advances and Applications, vol. 93, Birkhäuser Verlag, Basel, 1997.

[6] G.I. Eskin, Boundary value problems for elliptic pseudodifferential equations, Math. Monographs, vol. 52, Amer. Math. Soc., Providence, Rhode Island, 1980, Transl. of Nauka, Moskva, 1973.

[7] J.B. Gil, T. Krainer, and G. Mendoza, Geometry and Spectra of Closed Extensions of Elliptic Cone Operators, Preprint 2004/21, Institut für Mathematik, Potsdam, 2004.

[8] J.B. Gil, T. Krainer, and G. Mendoza, Resolvents of Elliptic Cone Operators, Preprint 2004/22, Institut für Mathematik, Potsdam, 2004.

[9] I. Gohberg and N. Krupnik, The algebra generated by the one-dimensional singular integral operators with piecewise continuous coefficients, Funk. Anal. i Prilozen. 4, 3 (1970), 26-36.

[10] I.C. Gohberg and E.I. Sigal, An operator generalization of the logarithmic residue theorem and the theorem of Rouché, Math. USSR Sbornik 13, 4 (1971), 603-625.

[11] G. Grubb, Functional calculus of pseudo-differential boundary problems. Second Edition, Birkhäuser Verlag, Boston, 1996. 
[12] G. Harutjunjan and B.-W. Schulze, Boundary problems with meromorphic symbols in cylindrical domains, Preprint 2004/12, Institut für Mathematik, Potsdam, 2004.

[13] D. Kapanadze and B.-W. Schulze, Crack theory and edge singularities, Kluwer Academic Publ., Dordrecht, 2003.

[14] V.A. Kondratyev, Boundary value problems for elliptic equations in domains with conical points, Trudy Mosk. Mat. Obshch. 16 (1967), 209-292.

[15] V.A. Kondratyev and O.A. Oleynik, Boundary problems for partial differential equations on non-smooth domains, Uspekhi Mat. Nauk 38, 2 (1983), 3-76.

[16] T. Krainer, On the inverse of parabolic boundary value problems for large times, Japan. J. Math. 30, 1 (2004), 91-163.

[17] T. Krainer and B.-W. Schulze, Long-time asymptotics with geometric singularities in the spatial variables, Contemporary Mathematics 364 (2004), $103-126$.

[18] R. Lauter and V. Nistor, Analysis of geometric operators on open manifolds: a groupoid approach, Quantization of Singular Symplectic Quotients (N. Landsman, M. Pflaum, and M. Schlichenmaier, eds.), Progress in Mathematics, vol. 198, Birkhäuser, Basel-Boston-Berlin, 2001, pp. 181-229.

[19] X. Liu and B.-W. Schulze, Boundary value problems in edge representation, Preprint 2004/14, Institut für Mathematik, Potsdam, 2004.

[20] P. Loya, Index theory of Dirac operators on manifolds with corners up to codimension two, Advances in Partial Differential Equations (Aspects of boundary problems in analysis and geometry) (J. Gil, T. Krainer, and I. Witt, eds.), Oper. Theory Adv. Appl., Birkhäuser Verlag, Basel, 2004, pp. 131-169.

[21] V.G. Maz'ja and B.A. Plamenevskij, $L_{p}$ estimates for solutions of elliptic boundary value problems in domains with edges, Trudy Mosk. Mat. Obshch. 37 (1978), 25-82, Engl. Transl.: Amer. Math. Soc. Transl. 123 (1984), 1-56.

[22] V.G. Maz'ja and J. Rossmann, Über die Asymptotik der Lösungen elliptischer Randwertaufgaben in der Umgebung von Kanten, Math. Nachr. 138 (1988), $27-53$.

[23] V. Nazaikinskij, A. Savin, B.-W. Schulze, and B. Ju. Sternin, Elliptic theory on manifolds with nonisolated singularities: II. Products in elliptic theory on manifolds with edges, Preprint 2002/15, Institut für Mathematik, Potsdam, 2002. 
[24] V. Nazaikinskij, A. Savin, B.-W. Schulze, and B. Ju. Sternin, Elliptic theory on manifolds with nonisolated singularities: III. The spectral flow of families of conormal symbols., Preprint 2002/20, Institut für Mathematik, Potsdam, 2002.

[25] V. Nazaikinskij, A. Savin, B.-W. Schulze, and B. Ju. Sternin, On the homotopy clssification of elliptic operators on manifolds with edges., Preprint 2004/16, Institut für Mathematik, Potsdam, 2004.

[26] V. Nazaikinskij, B.-W. Schulze, and B. Ju. Sternin, Surgery and the relative index theorem for families of elliptic operators., Preprint 2002/11, Institut für Mathematik, Potsdam, 2002.

[27] V. Nazaikinskij and B. Ju. Sternin, The index locality principle in elliptic theory, Funct. Anal. and its Appl. 35 (2001), 37-52.

[28] V. Nistor, Higher index theorems and the boundary map in cyclic homology, Documenta 2 (1997), 263-295.

[29] B.A. Plamenevskij, Algebras of pseudo-differential operators, Nauka, Moscow, 1986.

[30] S. Rempel and B.-W. Schulze, Index theory of elliptic boundary problems, Akademie-Verlag, Berlin, 1982.

[31] E. Schrohe and B.-W. Schulze, Boundary value problems in Boutet de Monvel's calculus for manifolds with conical singularities I, Advances in Partial Differential Equations (Pseudo-Differential Calculus and Mathematical Physics), Akademie Verlag, Berlin, 1994, pp. 97-209.

[32] E. Schrohe and B.-W. Schulze, Boundary value problems in Boutet de Monvel's calculus for manifolds with conical singularities II, Advances in Partial Differential Equations (Boundary Value Problems, Schrödinger Operators, Deformation Quantization), Akademie Verlag, Berlin, 1995, pp. 70-205.

[33] E. Schrohe and B.-W. Schulze, A symbol algebra for pseudodifferential boundary value problems on manifolds with edges, Differential Equations, Asymptotic Analysis, and Mathematical Physics, Math. Research, vol. 100, Akademie Verlag, Berlin, 1997, pp. 292-324.

[34] B.-W. Schulze, Pseudo-differential operators on manifolds with edges, Symposium "Partial Differential Equations", Holzhau 1988, Teubner-Texte zur Mathematik, vol. 112, Teubner, Leipzig, 1989, pp. 259-287.

[35] B.-W. Schulze, Operators with symbol hierarchies and iterated asymptotics, Publications of RIMS, Kyoto University 38, 4 (2002), 735-802. 
[36] B.-W. Schulze, Toeplitz operators, and ellipticity of boundary value problems with global projection conditions., Advances in Partial Differential Equations (Aspects of boundary problems in analysis and geometry) (J. Gil, T. Krainer, and I. Witt, eds.), Oper. Theory Adv. Appl., Birkhäuser Verlag, Basel, 2004, pp. 342-429.

[37] B.-W. Schulze and N.N. Tarkhanov, Euler solutions of pseudodifferential equations, Integral Equations Operator Theory 33 (1999), 98-123.

[38] M.I. Vishik and G.I. Eskin, Convolution equations in a bounded region, Uspekhi Mat. Nauk 20, 3 (1965), 89-152.

[39] M.I. Vishik and G.I. Eskin, Convolution equations in bounded domains in spaces with weighted norms, Mat. Sb. 69, 1 (1966), 65-110. 